DOE-ER40757-004

CPP-93-04

March 1993

\title{
The Geometric Phase in Quantum Physics
}

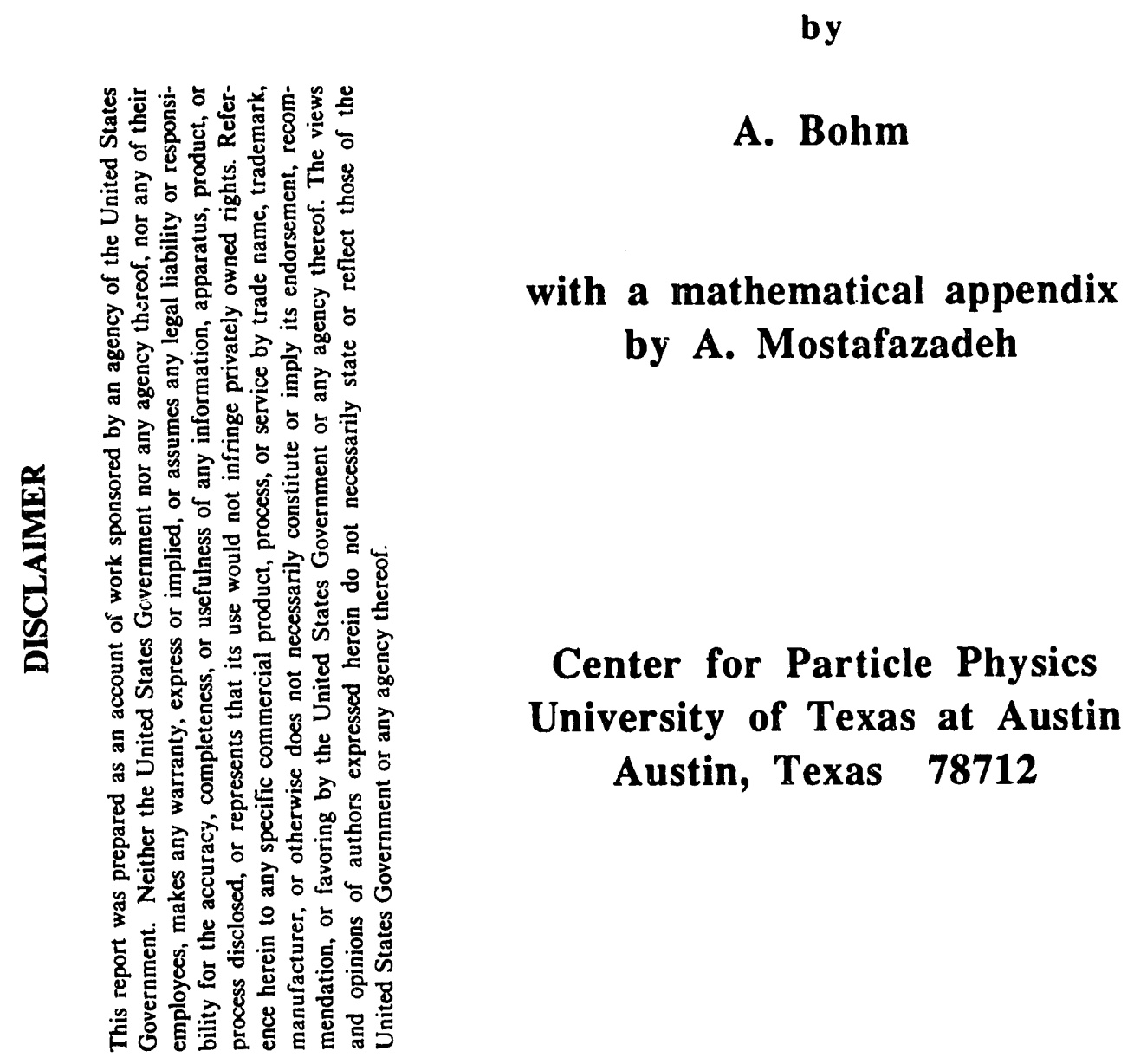

Lectures at the NATO Summer Institute, Salamanca, 1992. To be published in Recent Problems in Mathematical Physics, I. Ibort and M. A. Rodriguez (editors), Kluwer Academic Publishers (1993)

\section{MASTER}


The Geometric Phase in Quantum Physics

Contents

l. Introduction . . . . . . . . . . . . . . . . . . . . . . 1

2. Quantal Phase Factors for Adiabatic Changes . . . . . . . . . . 5

3. A Spinning Quantum System in an External Magnetic Field . . . 19

3.1. The Parameterization of the Basis Vectors . . . . . . . . . 19

3.2. Berry Connection and Berry Phase for Adiabatic Evolutions Magnetic Monopole Potentials . . . . . . . . . . . . . . . . 24

3.3. The Exact Solution of the Schrödinger Equation . . . . . . . 30

3.4. The Dynamical and the Geometrical Phase Factor for Non-Adiabatic Evolution . . . . . . . . . . . . . . . . . . . 34

4. The Anandan-Aharonov Phase for General Cyclic Evolution and its Relation to Berry's Phase - $U(1)$ Bundles Over Parameter Space and Over the Space of Physical States . . . 38

Acknowledgement . . . . . . . . . . . . . . 57

Mathematical Appendix: A Brief Review of Fibre Bundles and Their Classification by Ali Mostafazadeh . . . . . . . . . . . . 58 


\title{
THE GEOMETRIC PHASE II' QUANTUM PHYSICS*
}

\author{
A. BOHM \\ The Physics Department \\ The University of Texas \\ Austin, Texas 78712-1081, USA
}

\section{Introduction}

The geometrical phase has been ignored in quantum physics for half a century. It had not been forgotten, but it had been shown by V. Fock in $1928^{1)}$ that the extra phase factor which occurs for time-dependent Hamiltonians can be chosen to unity. Though Fock's proof was limited to non-cyclic evolution, this phase choice was generally used until around 1980 when, Mead et al. and Berry reconsidered cyclic evolutions.

Cyclic evolutions can occur if one considers a quantum physical system in a periodically changing environment. This environment can be classical (magnetic moment in a precessing external magnetic field) or quantal (a fast moving electron in the slowly changing quantal environment given by the collective motion of the molecule as a whole). The importance of the quantum geometric phase and the gauge potential connected with it was discovered in 1978 in molecular physics by Mead et $\mathrm{al}^{2}$ ) based upon some earlier observations by Herzberg and Longuet-Higgins. ${ }^{4}$ ) The motion of the molecule is naturally divided into two "parts", the fast motion of the electrons and the slow collective vibrations and rotations of the molecule as a whole. ${ }^{3)}$ In the past this problem was treated in the following way: One first investigated the motion of the fast variables considering the slow variables as fixed parameters. After the dynamics of the fast variables has been solved for all values of these fixed parameters, one turns to the dynamics of the slow variables. In this treatment the division into the slow and the fast moving parts is trivial. These are the ideas that underly the molecular Born-Oppenheimer approximation. ${ }^{5}$ If one does not consider the nuclear coordinates $R$ as fixed parameters but as quantum observables whose (eigen)-values change (e.g. rotate) in time, then the gauge potential emerges naturally from the Born-Oppenheimer method. ${ }^{3)}$

Conceptually simpler is the discussion of Hamiltonians which depend upon slowly changing parameters. This was done in 1983 in a beautiful paper by M.V. Berry ${ }^{6}$ ) for any quantum system in a slowly changing ("adiabatic") classical environment. Independently he arrived at the same ("Berry") gauge potentials and ("Berry") phase that were derived from the Born-Oppenheimer method by Mead. ${ }^{7)}$

It is easy to show in general that exact adiabatic cyclic evolutions do not exist; ${ }^{8)}$ the Berry phase (and the Berry potential) is only an approximation of the geometric phase of real cyclic evolutions. This quantum geometric phase was introduced by Anandan and Aharonov in $1987 .{ }^{9)}$

The geometric phase has observable consequences in many problems of physics and chemistry. ${ }^{10)}$ This is one reason why it has become an important subject. But the geometric phase is also one of the beautiful examples of what Wigner called "the unreasonable effectiveness of mathematics in natural sciences." Immediately after Berry

* Lectures at the NATO-ASI on Recent Problems in Mathematical Physics, Salamanca, Spain, June, 1992. 
introduced his phase Simon ${ }^{11)}$ noticed that it is the holonomy of fibre bundle and that the gauge potential is the connection of this bundle. And it was this relation to the beautiful mathematics of fibre bundles which caused the geometric phase to become a fashion in mathematical physics. When fibre bundles were created and when the mathematics of the universal classifying bundles was developed, one had no idea that this could have anything to do with a quantum mechanical phase factor which could be measured in an interference experiment. The Stiefel connection of the universal classifying bundle was the "natural" mathematical object to define. It is incredible that this mathematical entity is exactly the vector potential whose integral over a closed path of states gives the Anandan-Aharonov ${ }^{9)}$ phase with the Berry ${ }^{7}$ potential as its limiting case and that this connection is related to the vector potential which would be discovered by $\mathrm{Mead}^{2}$ ) in the molecular structure.

In addition to its usefulness for physical applications and its attractiveness for the mathematical formulation, the geometric phase also teaches us something new about the meaning of understanding in science.

Understanding means the separation of complicated physical systems into simpler subsystems, the parts. In classical physics, separation into parts usually means reduction to the simpler objects, the constituents. In quantum mechanics the "parts" are described by subspaces of the Hilbert space ${ }^{12)}$ though these subspaces do not have to represent constituents (they may, e.g., represent collective vibrations or rotations as in the BornOppenheimer method). ${ }^{5}$ ) The dissection of complicated quantum systems results in the trivial direct product of the states for the subsystems and the combinaton of two parts is given by the direct product of the Hilbert spaces for the parts (if the parts are (identical) particles the many body Hilbert space is the (symmetrized or antisymmetrized) tensor product of the one-particle Hilbert spaces).

In contrast to this quantum mechanical hypothesis, the geometrical phase - if it is non-trivial - shows that a part of the complicated physical system is not what one trivially expects. The fast motion effects the slow moving part and modifies its dynamics such that the many body Hilbert space is not always the tensor product of the parts.

In this review we shall discuss only quantum systems in a classical time-dependent environment. Quantum systems in a changing quantal environment, that appear in the dissection of complicated quantum systems into their parts are discussed in a number of publications. ${ }^{2)}$ 3) With a few exceptions ${ }^{13)}$ they are restricted to molecular physics. ${ }^{14)}$

Our presentation in sections 2 and 3 does not make use of differential geometric notions, like fibre bundles, but it uses some of its nomenclature. Section 2 is restricted to the adiabatic approximation and discusses the general abelian case. In section 3 we discuss the standard example in detail but in greater generality than usual. We start with the adiabatic approximation in section 3.2 and then introduce the geometric phase for non-adiabatic change of the environment (Anandan-Aharonov phase). ${ }^{*}$ In section 4 we discuss general cyclic (non-adiabatic) evolution. We introduce the mathematics of fibre bundles and use some of its results to describe the relation between the adiabatic Berry phase and the geometric phase for general cyclic evolution of a pure state. In this

* Sections 2 and 3 are based on Chapter XXII of the 3rd edition of the book in reference 14 , which contains details of the calculations. 
review we restrict ourselves to the abelian, $U(1)$ phase. These results generalize in a straightforward way ${ }^{8)}$ to states with $\mathcal{N}$-fold degeneracy (using a $U(\mathcal{N})$ phase $^{15)}$ ). Some features of these concepts also extend to cyclic evolutions of states described by density
operators. ${ }^{16)}$ 
1) V. Fock. Z. Phys. 49, 323 (1928).

\section{References}

2) C. Mead and D. Truhlar, J. Chem. Phys. 70, 2284 (1979); C.A. Mead, Chem. Phys. 49, 23 and 33 (1980).

3) J. Moody, A. Shapere, F. Wilczek, Phys. Rev. Lett. 56, 893 (1986); R. Jackiw, Int. J. Mod. Phys. A3, 285 (1988); A. Bohm, Lectures on Symmetry in Science III, 1988; p. 85, B. Gruber and F. Iachello (editors) Plenum Press; B. Zygelman, Phys. Rev. Lett. 64, 256 (1990).

4) G. Herzberg and H.C. Longuet-Higgins, Discuss, Faraday Soc. 35, 77 (1963); H.C. Longuet-Higgins, Proc. Roy. Soc. London Ser. A344, 147 (1975).

5) M. Born and J. Oppenheimer, Ann. Phys. 84, 457 (1927).

6) M.V. Berry, Proc. Roy. Soc. London Ser. A392, 45 (1984).

7) For earlier "anticipations" of the Berry phase, see M. Berry, Physics Today, December, 1990, p. 34.

8) A. Bohm, The Berry Connection as a Limit of the Stiefel Connection, Proceedings of the XIX International Group Theory Colloquium, Salamanca (1992).

9) Y. Aharonov and J. Anandan, Phys. Rev. Lett. 58, 1593 (1987); J. Anandan and Y. Aharonov, Phys. Rev. D38, 1863 (1988).

10) A. Shapere, F. Wilczek, Geometric Phases in Physics (Review and Reprint volume), World Scientific (1989); J. Zwanziger, M. Koenig, A. Pines, Berry's Phase, Annual Rev. Phys. Chem., 41, 601 (1990); J.W. Zwanziger, S.P. Rucher, G.C. Chingas, Phys. Rev. A43, 3232 (1991).

11) This phase was shown to be the holonomy of a fibre bundle with Berry connection; B. Simon, Phys. Rev. Lett. 51, 2167 (1983).

12) More precisely, of the space of physical states $\Phi$ which is a subspace of the Hilbert space, A. Bohm, M. Gadella, Dirac Kets, Garnow Vectors and Gelfand Triplets, Springer-Verlag, Berlin (1989).

13) A. Bulgac, Phys. Rev. C41, 2333, (1990); R.S. Nikam, P. Ring, Y. Sun, E.R. Marshalek, Phys. Lett. 235B, 215, (1990). M. Rho, International Workshop on Baryons as Skyrme Solitons, Siegen, September 1992.

14) There exist a few reviews and expositions of this subject: C. Alden Mead, The Geometric Phase in Molecular Systems. Reviews Modern Physics 64, 51 (1992); Chapter XXIII, Third Edition of A. Bohm, Quantum Mechanics: Foundations and Applications to appear in Springer-Verlag, New York, 1993/94.

15) F. Wilczek, A. Zee, Phys. Rev. Lett. 52, 2111 (1984).

16) A. Uhlmann, Parallel Transport of Phases. Lecture Notes of Physics, Vol. 379, 55, Springer-Verlag (1991). 


\section{Quantal Phase Factors for Adiabatic Changes}

The observables of a quantum system which is not isolated from its environment are described by operators that depend upon parameters $R=(x, y \ldots)$. We assume that the parameter space is a differentiable manifold and the observables, in particular the Hamiltonian $h(R)$, are nice (continuous, infinitely differentiable) and single-valued functions on the parameter space $M$. These parameters $R$ (which we also write as $\mathbf{R}$ if we want to suggest that it is a three-vector) describe the classical environment in which the quantum physical system is immersed. If the physical system is in a quantal environment then the parameters are (generalized) eigenvalues of the observables of the quantal environment (like e.g. the position operator of the internuclear axis of a diatomic molecule).

An example of a quantum physical system whose Hamiltonian depends upon environmental parameters is a (quantum) magnetic moment $\mathbf{m}$ in a rotating (classical) magnetic fieid $\mathbf{B}$ of constant magnitude $B \equiv|\mathbf{B}|$. The parameter dependent Hamiltonian is giver. according to (IX.3.1) ${ }^{1)}$ by

$$
H=H_{0}-\mathbf{m} \cdot \mathbf{B}=H_{0}-B g \frac{e}{2 m c} \hat{\mathbf{R}} \cdot \mathbf{J}=H_{0}+b \hat{\mathbf{R}} \cdot \mathbf{J}
$$

Here $H_{0}$ is the Hamiltonian without the magnetic field, which we shall ignore. The quantum system's magnetic moment is given in terms of its angular momentum operator $\mathbf{J}$ according to (IX.3.18) $)^{1)}$ by

$$
\mathbf{m}=+\frac{e}{2 m c} g \mathbf{J},
$$

$\hat{R}(t)=\mathrm{B} / B$ is the unit vector pointing in the direction of the magnetic field, and $b=-B g\left(\frac{e}{2 m c}\right)$ is a constant. The parameter dependent term of the Hamiltonian is $h(\hat{\mathbf{R}})=b \hat{\mathbf{R}} \cdot \boldsymbol{J}$ and the parameter space of the environment is the unit sphere $\{\hat{\mathbf{R}} ;|\hat{\mathbf{R}}|=$ $1\}$. We can use as the parameters the polar angles $\hat{\mathbf{R}}=(\theta, \phi)$ of the unit vectors $\hat{\mathbf{R}}$.

The evolution of states of the quantum system in the external environment is described by the Schrödinger equation

$$
i \frac{d \psi(t)}{d t}=h(R) \psi(t)
$$

(if the state is pure and described by a vector $\psi(t)$, and for general states described by the statistical operator $W(t)$ the evolution is given by the Schrödinger-von Neumann equation

$$
i \frac{d W(t)}{d t}=[h(R), W(t)]
$$

This is the generalization of a basic postulate of quantum mechanics for conservative systems. ${ }^{2)}$ The evolution of states of the non-conservative system is determined by the dynamical equation (2.3) and by the environmental process, i.e., by the way in which the environmental parameters $\mathbf{R}$ change. For a given physical situation, the way in which the parameters change, i.e., the path in the environment's parameter space, must be specified. In specifying the environmental process it is usually convenient to give the environmental parameters a time parameterization $\mathbf{R}(t)=(x(t), y(t), \cdots)$. One then obtains from $h(\mathbf{R})$ a time-dependent Hamiltonian $h(t)=h(\mathbf{R}(t))$. In our example 
(2.1) the environmental processes are described by how the direction $\hat{\mathbf{R}}$ of the magnetic field changes. The direction of the magnetic field may change periodically; e.g., it may perform rotations in the 1-2 plane with angular velocity $\omega, \hat{\mathbf{R}}(t)=\mathbf{e}_{1} \cos \omega t-\mathbf{e}_{2} \sin \omega t$, or it may run through other closed paths $\mathbf{C}$ in the parameter space. In this case the Hamiltonian $h(t)=h(\hat{\mathbf{R}}(t))$ returns to its original form as time progresses from $t=0$ to the period $t=T=2 \pi / \omega$.

We postulate that the space of physical states does not only contain the solutions of (2.3) for one given fixed value of the parameter $\mathbf{R}$ or for one given environmental process $t \rightarrow \mathbf{R}(t)$ but for all values $\mathbf{R} \in$ parameter space $M$. This means that there is one space of physical states $\mathcal{H}$ for all values of $\mathbf{R}$. For any given value of $\mathbf{R}$, one may choose an orthonormal basis of eigenvectors $|n ; \mathbf{R}\rangle$ of the parameter dependent Hamiltonian $h(\mathbf{R}),^{*}$

$$
\begin{aligned}
h(\mathbf{R})|n ; \mathbf{R}\rangle & =E_{n}(\mathbf{R})|n ; \mathbf{R}\rangle \\
\langle m ; \mathbf{R} \mid n ; \mathbf{R}\rangle & =\delta_{m, n},
\end{aligned}
$$

and write the Hamiltonian according to its spectral resolution (I.4.10d): ${ }^{1)}$

$$
h(\mathbf{R})=\sum_{n} E_{n}(\mathbf{R})|n ; \mathbf{R}\rangle\langle n ; \mathbf{R}|
$$

Given any environmental process along with a time parameterization $\mathbf{R}(t)$ one obtains from the parameter dependent Hamiltonian $h(\mathbf{R})$ in (2.5) a time-dependent Hamiltonian $h(t)=h(\mathbf{R}(t))$ along with its spectral resolution (of I.4.10d):

$$
h(\mathbf{R}(t))=\sum_{n} E_{n}(\mathbf{R}(t))|n ; \mathbf{R}(t)\rangle\langle n ; \mathbf{R}(t)|
$$

The projection operators

$$
\Lambda_{n}(\mathbf{R}(t)) \equiv|n ; \mathbf{R}(t)\rangle\langle n ; \mathbf{R}(t)|
$$

change in general with time.

We have assumed that the observables are single-valued as functions of $\mathbf{R}$ over the whole parameter space of the environment. Single-valuedness of the observables means that if the same value of $\mathbf{R}$ occurs more than once (i.e., at different times) during a process, then the observables are the same at each occurence. In particular, if the environmental process is closed, i.e., if the environmental parameters $\mathbf{R}(t)$ traverse a closed path $\mathbf{C}$ and return, after some period $T$, to their original values,

$$
\mathbf{C}: \mathbf{R}(0) \rightarrow \mathbf{R}(t) \rightarrow \mathbf{R}(T)=\mathbf{R}(0)
$$

* We assume here, for simplicity, that the spectrum of $h(\mathbf{R})$ is discrete. If in addition to $h(\mathbf{R})$ other observables $A_{i}(\mathbf{R}), i=1, \cdots, N-1$ are needed to form a c.s.c.o. for any given value of $\mathbf{R}$, then their eigenvalues $a_{i}(\mathbf{R})$ will also be needed to label the basis vectors $|n ; \mathbf{R}\rangle=\left|n, a_{1}, \cdots, a_{N-1} ; \mathbf{R}\right\rangle$. 
then the Hamiltonian and also its eigenvalues and projection operators, which are uniquely defined by (2.5), are the same at $\mathbf{R}(T)$ as they are at $\mathbf{R}(0)$ :

$$
\begin{aligned}
h(\mathbf{R}(T)) & =h(\mathbf{R}(0)) \\
E_{n}(\mathbf{R}(T)) & =E_{n}(\mathbf{R}(0)) \\
|n ; \mathbf{R}(T)\rangle\langle n ; \mathbf{R}(T)| & =|n ; \mathbf{R}(0)\rangle\langle n ; \mathbf{R}(0)|
\end{aligned}
$$

Though the observables are single-valued functions of $\mathbf{R}$ the basis vectors $|n ; \mathbf{R}\rangle$ themselves will in general not be single-valued functions of $\mathbf{R}$ over the whole parameter space. Usually it is necessary to use different parameterization over different patches of the parameter space. For the example $h(\mathbf{R})=b \mathbf{R}(\theta, \varphi) \cdot \mathbf{J}$ this means that the vectors in, $\mathbf{R}(\theta, \varphi)\rangle$ are different functions of the polar angles $(\theta, \varphi)$ for different patches of the unit sphere, as explained in detail in section 3, (cf. equations (3.11) and (3.19)). Thus in general (2.10) will not imply

but only

$$
|n ; \mathbf{R}(T)\rangle=|n ; \mathbf{R}(0)\rangle \quad \text { for } \quad \mathbf{R}(T)=\mathbf{R}(0)
$$

$$
|n ; \mathbf{R}(T)\rangle=e^{i \zeta_{n}}|n ; R(0)\rangle \quad \text { for } \quad \mathbf{R}(T)=R(0)
$$

where $e^{i \zeta_{n}}$ is a phase factor, because the $|n ; \mathbf{R}\rangle$ are determined by (2.4) only up to a phase factor. We can define a new system of eigenvector $|n ; \mathbf{R}\rangle$ by making phase transformations (also called gauge transformations as shall be explained below),

$$
|n ; \mathbf{R}\rangle \quad \rightarrow \quad|n ; \mathbf{R}\rangle^{\prime}=e^{i \zeta_{n}(\mathbf{R})}|n ; \mathbf{R}\rangle
$$

where the $\zeta_{n}(\mathbf{R})$ are arbitrary real phase angles. Any such basis constitutes just as valid a basis of eigenvectors of the Hamiltonian as the $|n ; \mathbf{R}\rangle$.

We will restrici ourselves to transformations for which the phase factors $e^{i \zeta_{n}(\mathbf{R}(t))}$ are single-valued functions. Only these are called gauge transformations.

In general, if we go from one patch $O_{1}, \subset M$ of the parameter space into a neighboring patch $\mathrm{O}_{2} \subset M$ with a different parameterization, then eigenvectors of $h(R)$ in the overlap region $R \in O_{1} \cap O_{2}$ are related by the gauge transformation (2.12). We will assume however that the closed path $\mathbf{C}$ of (2.7) can always be placed into one single patch $O \subset M$ and the basis vectors can be chosen to be single-valued functions. We shall then choose for the basis vectors single-valued functions over parameter space so that (2.11) is fulfilled. The gauge transformation (2.12) will transform single-valued basis vectors into vectors which are also single valued.

So far we considered the observables (the $|n ; \mathbf{R}(t)\rangle$ are also observables), which change in time due to the change of the environmental parameters. We now want to consider the states $W(t)$, or the pure states $|\psi(t)\rangle\langle\psi(t)|$ (which can also be described by the state vector $\psi(t))$. The states evolve in time due to the Schrödinger or von Neumann equation (2.3).

The time evolution can also be described by an operator $U^{i}(t)$ (which is unitary dus to the hermiticity of $h(\mathbf{R}(t))=h(t))$

$$
\psi(t)=U^{\dagger}(t) \psi(0)
$$


which fulfills as a consequence of (2.3) the integral equation

$$
U^{\dagger}(t)=I-i \int_{0}^{t} h\left(t^{\prime}\right) U^{\dagger}\left(t^{\prime}\right) d t^{\prime}
$$

An expression for $U^{\dagger}(t)$ in terms of $h(t)$ is then given by successively substituting the right-hand side of (2.14) for the $U^{\dagger}(t)$ that appears in the integrand:

$$
\begin{aligned}
U^{\dagger}(t)= & +\frac{1}{i} \int_{0}^{t} d t^{\prime} h\left(t^{\prime}\right)+\left(\frac{1}{i}\right)^{2} \int_{0}^{t} d t^{\prime} \int_{0}^{t^{\prime}} d t^{\prime \prime} h\left(t^{\prime}\right) h\left(t^{\prime \prime}\right) U^{\dagger}\left(t^{\prime \prime}\right) \\
= & I+\frac{1}{i} \int_{0}^{t} d t^{\prime} h\left(t^{\prime}\right)+\left(\frac{1}{i}\right)^{2} \int_{0}^{t} d t^{\prime} \int_{0}^{t^{\prime}} d t^{\prime \prime} h\left(t^{\prime}\right) h\left(t^{\prime \prime}\right)+\cdots \\
& +\left(\frac{1}{i}\right)^{n} \int_{0}^{t} d t_{1} \int_{0}^{t_{1}} d t_{2} \cdots \int_{0}^{t_{n-1}} d t_{n} h\left(t_{1}\right) h\left(t_{2}\right) \cdots h\left(t_{n}\right)+\cdots
\end{aligned}
$$

Note that the operators appearing in the integrands have decreasing time arguments reading from left to right, $t \geq t^{\prime} \geq t^{\prime \prime} \geq 0 ; t \geq t_{1} \geq t_{2} \geq \ldots \geq t_{n} \geq 0$, and that their order is important since they do not in general commute:

$$
\left[h(t), h\left(t^{\prime}\right)\right] \neq 0 \quad \text { for } t^{\prime} \neq t .
$$

For the case that $h(t)$ commutes at different times,

$$
\left[h(t), h\left(t^{\prime}\right)\right]=0 \quad \text { for all } t, t^{\prime},
$$

the order of the operators in (2.15) is not important and it can be shown (Problem 2.1)*

$$
U^{\dagger}(t)=\sum_{n=0}^{\infty} \frac{1}{n !}\left(\frac{1}{i}\right)^{n} \int_{0}^{t} d t_{1} \ldots \int_{0}^{t} d t_{n} h\left(t_{1}\right) \ldots h\left(t_{n}\right)=e^{\frac{1}{t} \int_{0}^{t} d t^{\prime} h\left(t^{\prime}\right)} .
$$

According to (II.4.45a), ${ }^{1)}$ (2.17) means that the projection operators $\Lambda_{n}(\mathbf{R}(t))=|n ; \mathbf{R}(t)\rangle\langle n ; \mathbf{R}(t)|$ commute:

$$
\left[\Lambda_{n}(\mathbf{R}(t)), \Lambda_{n^{\prime}}\left(\mathbf{R}\left(t^{\prime}\right)\right)\right]=0 \quad \text { for all } n, n^{\prime} \text { and } t, t^{\prime}
$$

Together with the assumed continuity of the $\Lambda_{n}(\mathbf{R}(t))$ this means that the projection operators and eigenspaces of $h(t)$ are in fact time-independent witis only the eigenvalues $E_{n}(t)$ depending upon time. The projection operators may then be written as $\Lambda_{n}(\mathbf{R}(t))=\Lambda_{n}=|n\rangle\langle n|$ with time-independent vectors $|n\rangle$. The resulting spectral resolution (2.6) of $h(t)$ may then be used in (2.18) to give the following spectral resolution of $U^{\dagger}(t)$ :

$$
U^{\dagger}(t)=\sum_{n} e^{t} \int_{0}^{t} d t^{\prime} E_{n}\left(t^{\prime}\right)|n\rangle\langle n|
$$

\footnotetext{
* Problem 2.1. Show that (2.18) is obtained from (2.15) if (2.17) holds (cf. e.g. reference 3).
} 
(If the eigenvalues are also independent of time, i.e., if the Hamiltonian is time-independent, $h(t)=h$, then (2.19) goes into the standard expression for conservative systems (XII.1.16) $\left.{ }^{1)} U^{\dagger}(t)=e^{\frac{t}{t} t h}\right)$. But for general time-dependent Hamiltonians, if (2.16) holds, (2.14) cannot be integrated. (Formally one obtains the time-ordered product of the expression on the right-hand side of $(2.18)^{3)}$ but this is not of much practical value).

We now solve (2.3) under various approximations. We use the initial condition that at $t=0$ the state is an eigenstate of $h(R(0))$ with energy $E_{n}(R(0))$ which means

$$
|\psi(0)\rangle\langle\psi(0)|=| n ; R(0)\rangle\langle n ; R(0)| \quad \text { or } \quad \psi(0)=|n ; R(0)\rangle
$$

The second form of this initial condition follows after fixing an arbitrary phase factor to unity. First we consider $R=$ "fixed" parameter (This is the assumption that has been used for the old Born-Oppenheimer approximation in molecular physics). Then the solution of $(2.3)$ with $(2.21)$ is

$$
\psi(t)=e^{-i E_{n}(R) t}|n ; R\rangle=e^{-i E_{n}(R) t} \psi(0)
$$

Essentially the same result we obtain if $R(t)$ changes, but only in such a way that $(2.17)$ is fulfilled. Then (2.20) with the initial condition (2.21) gives immediately:

$$
\psi(t)=e^{-i \int_{0}^{t} d t^{\prime} E_{n}\left(R\left(t^{\prime}\right)\right)}|n ; R(0)\rangle=e^{-i \int_{0}^{t} d t^{\prime} E_{n}\left(R\left(t^{\prime}\right)\right)} \psi(0)
$$

We now consider the less drastic assumption, called the adiabatic approximation. ${ }^{4}$ For a Hamiltonian $h(\mathbf{R}(t))$ whose parameter change in time the interaction with the environment can cause the physical system to jump from the $\mathrm{n}$-th eigenstate at $t=0$ into all other eigenstates $|m, \mathbf{R}(t)\rangle\langle m, \mathbf{R}(t)|, m \neq n$ at a later time $t$. A very particular situation arises if this is not the case and if the state remains an eigenstate of $h(\mathbf{R}(t))$ at all time $t$ with the same energy quantum number $n$. This means that $|\psi(t)\rangle\langle\psi(t)|$ changes in such a way that at all times $t$

$$
|\psi(t)\rangle\langle\psi(t)|\stackrel{\text { adiabatic }}{\stackrel{\perp}{=}}| n ; \mathbf{R}(t)\rangle\langle n ; \mathbf{R}(t)|=\Lambda_{n}(\mathbf{R}(t))
$$

This time-development is called adiabatic time-development. It represents an approximation not a limiting case. States which do not change in time, i.e. for which

$$
|\psi(t)\rangle\langle\psi(t)|=| \psi(0)\rangle\langle\psi(0)| \quad \text { for all } t,
$$

are called stationary states. Clearly the solutions (2.22) and (2.23) describe stationary states:

$$
|\psi(t)\rangle\langle\psi(t)|=| n ; R(0)\rangle\langle n ; R(0)|
$$

One can show that stationary states of conservative systems are always energy eigenstates, cf. (XII.1.41)(XII.1.44). ${ }^{1}$ For general time-dependent Hamiltonians this is not the case. The issumption of adiabaticity (2.24) is a generalization of the assumption that the state is stationary (2.25). Because $\Lambda_{n}(R(t))$ changes in time, the adiabatic state $W(t)=|\psi(t)\rangle\langle\psi(t)|$ of (2.24) changes in time, whereas the stationary state (2.25) does not change. The state (2.24) will always be the n-th eigenstate of $h(R(t))$ but its eigenvalue $E_{n}(R(t))$ may change in time; and even if that happens to be not the case 
than $|n ; R(t)\rangle\langle n ; R(t)|$ can still change. The path $t \rightarrow|\psi(t)\rangle\langle\psi(t)|$ of $(2.24)$ describes a curve in the set of projection operators $|\psi(t)\rangle\langle\psi(t)|$ whereas the path of (2.25) consists of one point.

The set of one-dimensional projection operators $W(t)=|\psi(t)\rangle\langle\psi(t)|$ of the Hilbert space $\mathcal{H}$ is denoted by $\mathcal{P}(\mathcal{H})$. It is the projective space of (pure) physical states. The adiabaticity assumption (2.24) then means that if $R(t)$ traverses through a path in the parameter space $M$ then the pure state $|\psi(t)\rangle\langle\psi(t)|$ traverses through a path of Hamiltonian eigenstates $t \rightarrow \Lambda_{n}(\mathbf{R}(t))$ in $\mathcal{P}(\mathcal{H})$. In particular if $\mathbf{R}(t)$ traverses through the closed path $\mathbf{C}$ of (2.7) in the parameter space $M$ then because of (2.10) the pure Hamiltonian eigenstate traverses through a closed path

$$
\mathcal{C}: \Lambda_{n}(\mathbf{R}(0))=W(0) \rightarrow W(t) \rightarrow W(T) \stackrel{\text { adiabatic }}{=} \Lambda_{n}(R(T))=\Lambda_{n}(R(0))
$$

If the eigenprojectors traverse a closed path $\mathcal{C}$ in $\mathcal{P}(\mathcal{H})$ then the eigenvectors $|n ; \mathbf{R}(t)\rangle$ traverse a closed path in $\mathcal{H}$

$$
|n ; R(0)\rangle \rightarrow|n ; R(t)\rangle \rightarrow|n ; R(T)\rangle=|n ; R(0)\rangle
$$

because these basis vectors had been chosen to be single-valued functions in the patch that contained the closed curve $\mathbf{C}$ of $(2.7)$.

That $W(t)=|\psi(t)\rangle\langle\psi(t)|$ traverses a closed path in the state space $\mathcal{P}(\mathcal{H})$ does not necessarily mean that the normalized state vector $\psi(t)$, which fulfills the Schrödinger equation (2.3) also traverse a closed path in $\mathcal{H}$. In general the path

$$
C \quad: \quad t \rightarrow \psi(t) \quad 0 \leq t \leq T \quad ; \quad(\psi(t), \psi(t))=1
$$

is not closed in $\mathcal{H}$ but fulfills

$$
\psi(T)=e^{-i \alpha_{\psi}} \psi(0),
$$

For the case of a time-independent Hamiltonian (2.22) or for (2.23), the phase factor is:

$$
e^{-i \alpha_{\psi}}=e^{-i E_{n} T} \quad \text { or } \quad e^{-i \alpha_{\psi}}=e^{-i \int_{0}^{t} d t^{\prime} E_{n}\left(t^{\prime}\right)}
$$

which is called the dynamical phase factor.

We will instantly show that in case of a time-dependent Hamiltonian $h(\mathbf{R}(t))$ there is in general an additional phase factor which is called geometric phase for Berry phase (We shall in general use the term Berry phase for the geometrical phase obtained in the adiabatic approximation (2.24) only). But before we derive the geometric phase we want to discuss the meaning of the adiabatic approximation.

The dynamical equation (2.3) and the adiabaticity assumption (2.24) are two separate conditions on the state $|\psi(t)\rangle\langle\psi(t)|$ and may, therefore, not be compatible with each other. This is indeed the case, which can immediately be shown by inserting (2.24) into (2.3b):

$$
i \frac{d W(t)}{d t}=[h(R(t)), W(t)]=\left[h(R(t)), \Lambda_{n}(R(t))\right]=0
$$

which means that $W(t)$ does not change in time

$$
W(t) \equiv|\psi(t)\rangle\langle\psi(t)|=| \psi(0)\rangle\langle\psi(0)| \equiv W(0)
$$


This means that any adiabatic evolution (2.24) of a state (which obeys (2.3)) must be a stationary evolution (2.25) and cannot be a non-trivial cyclic evolution

$$
W(0) \rightarrow W(t) \rightarrow W(T)=W(0)
$$

for which $W(t)$ changes in time.

It is, of course, not clear that for a given Hamiltonian $h(\mathbf{R}(t))$ there exist at all a cyclic solution (2.34) of the dynamical equation (2.3). But it is plausible that for a periodic Hamiltonian, i.e. a time-dependent Hamiltonian fulfilling (2.8), there exist some (a countable set of) cyclic solutions fulfilling (2.34). (In section 3 we shall demonstrate this statement by an example). However, as we have seen from (2.32), these cyclic solutions cannot be Hamiltonian eigenstates $\Lambda_{n}(\mathbf{R}(t))$. Exact adiabatic cyclic evolutions do not adiabatic

exist. The adiabatic equality $\stackrel{\downarrow}{=}$ in (2.24) can only be an approximation, not a limiting case (except for $T \rightarrow \infty$ when the time-development becomes stationary and the evolution a trivial cyclic evolution (2.25)). Nevertheless we can use the approximation (2.24) and determine the adiabatic geometrical phase or Berry phase.

To do this we expand $\psi(t)$ with respect to the basis system $|n ; \mathbf{R}(t)\rangle$ :

$$
\psi(t)=\sum_{m} c_{m}(t)|m ; \mathbf{R}(t)\rangle=\sum_{m} a_{m}(t) e^{\frac{1}{i} \int_{0}^{t} E_{m}\left(t^{\prime}\right) d t^{\prime}}|m ; \mathbf{R}(t)\rangle .
$$

In the second equality we have separated the dynamical phase of the expansion coeffcients $c_{m}(t)$. For the special case (2.24) of the adiabatic approximation the expansion (2.35) becomes

$$
\psi(t) \stackrel{\text { adiabatic }}{\stackrel{⺊}{=}} c_{n}(t)|n, \mathbf{R}(t)\rangle=e^{\frac{1}{t} \int_{0}^{t} E_{n}\left(t^{\prime}\right) d t^{\prime}} a_{n}(t)|n, \mathbf{R}(t)\rangle
$$

where because of $(2.21)$

$$
a_{n}(0)=1
$$

This is not an equality but an approximate equality which we indicate by the qualification "adiabatic" at the equality sign. Inserting (2.36) into the Schrödinger equation (2.3) and using (2.4) we obtain after a little calculation

$$
\frac{d}{d t} a_{n}(t) \equiv \dot{a}_{n}(t)=-a_{n}\langle n ; \mathbf{R}(t)|\left(\frac{d}{d t}|n ; \mathbf{R}(t)\rangle\right) .
$$

This can be integrated:

$$
\int_{a_{n}(0)}^{a_{n}(t)} \frac{d a_{n}}{a_{n}}=-\int_{0}^{t}\left\langle n ; \mathbf{R}(t)\left|\frac{d}{d t}\right| n ; \mathbf{R}(t)\right\rangle d t
$$

and leads with (2.21') to:

$$
a_{n}(t)=e^{i \int_{\mathbf{R}(0)}^{\mathbf{R}(t)} i\left\langle\boldsymbol{n} ; \mathbf{R}(t)\left|\frac{\theta}{\partial \mathbf{R}^{i}}\right| \boldsymbol{n} ; \mathbf{R}(t)\right\rangle d R^{i}} \equiv e^{i \gamma_{n}(t)} .
$$

The coefficients $c_{n}(t)$ and $a_{n}(t)$ in (2.36) are phase factors (their absolute value is one), because $\psi(t)$ is obtained from $\psi(0)$ of $(2.21)$ by a unitary time-development $(2.13)$ and 
is therefore a normalized vector. This justifies the definition of $\gamma_{n}(t)$ as a real phase angle by (2.39). Note that $\gamma_{n}(t)$ is only defined up to a multiple of $2 \pi$.

The phase angle $\gamma_{n}(t)$ is defined in terms of an integral over a vector valued function

$$
\mathbf{A}^{n}(\mathbf{R}) \equiv i\langle n ; \mathbf{R}|\nabla| n ; \mathbf{R}\rangle
$$

which is called the Mead-Berry vector potential or the Mead-Berry connection. ${ }^{5}$ It is defined as the scalar product of the eigenvectors of $h(t)$ and its derivative with respect to the parameter $\mathbf{R}, \nabla|n ; \mathbf{R}\rangle$. For the definition (2.40) of $\mathbf{A}(\mathbf{R})$ one uses single valued basis vectors only. As a single-valued $|n ; \mathbf{R}\rangle$ can in general not be found on the whole parameter space but only on open subsets (patches) the same is true for the $A(\mathbf{R}){ }^{*}$ We will discuss this problem in detail for the example (2.1) in section 3. Here we will assume that the curve $\mathbf{C}$ lies in one of these patches and that the $|n ; \mathbf{R}\rangle$ are single-valued. The integral

$$
\begin{aligned}
\gamma_{n}(t) & =\int_{0}^{t} i\left\langle n ; \mathbf{R}(t)\left|\frac{d}{d t}\right| n ; \mathbf{R}(t)\right\rangle d t=\int_{R(0)}^{R(t)} i\left\langle n ; \mathbf{R}(t)\left|\frac{\partial}{\partial R^{i}}\right| n ; \mathbf{R}(t)\right\rangle d R^{i} \\
& =\int_{R(0)}^{R(t)} i\langle n ; \mathbf{R}(t)|d| n ; \mathbf{R}(t)\rangle=\int_{R(0)}^{R(t)} A_{i}^{n}(\mathbf{R}) d R^{i} \equiv \int A^{n}
\end{aligned}
$$

is independent of the parameterization as expressed by the third term of (2.41) as line integral of a differential along the curve $t \rightarrow \mathbf{R}(t)$.

For the sake of simplicity we have so far pretended that the environmental parameter is a three-vector $\mathbf{R}$, then a differential one-form is defined by

$$
A^{n} \equiv i\left\langle n ; R^{i}\left|\frac{\partial}{\partial R^{i}}\right| n ; R^{i}\right\rangle d R^{i}=A_{i}^{n} d R^{i}=i\left\langle n ; R^{i}|d| n ; R^{i}\right\rangle,
$$

where $A_{i}^{n}$ are the components of the three-vector (2.40). In general the parameter space is (assumed to be) a differentiable manifold and $R^{i} ; i=1,2, \ldots f ;$ are its arbitrary (local) coordinates. The differentials $d R^{i}$ are covariant vectors and form a basis dual to the basis $\frac{\partial}{\partial R^{i}}$ of contravariant vectors and $A^{n} \equiv i\langle n ; R|d| n ; R\rangle$ is a differential one-form independent of the parameterization. ${ }^{6)}$ The expressions for the phase angle $\gamma_{n}(t)$ thus apply also to an $f$-dimensional parameter space in which case the Berry vector potential $A_{i}^{n}$ is an $f$-dimensional contravariant vector.

Returning to our calculation of $\psi(t)$ we obtain by inserting (2.39) into (2.36) for the state vector in the adiabatic approximation with initial condition (2.21):

$$
\psi(t) \stackrel{\text { adiabatic }}{\stackrel{1}{=}} e^{\frac{1}{2} \int^{t} E_{n}\left(t^{\prime}\right) d t^{\prime}} e^{i \gamma_{n}(t)}|n ; \mathbf{R}(t)\rangle
$$

In addition to the dynamical phase factor (2.31) one obtains thus the phase factor $e^{i \gamma_{n}(t)}$ given in terms of the eigenvectors of $h(R(t))$ by $(2.41)$.

According to (2.12) the $|n ; R(t)\rangle$ are only determined up to a phase factor. Thus, the additional phase factor in (2.43) can be transformed away by a phase transformation.

\footnotetext{
* It is, therefore, also called a local representative of the Mead-Berry connection.
} 
From the requirement that the (eigen)vectors $\psi(t)$ have the least possible oscillatory behavior, $\left\|\frac{d \psi(t)}{d t}\right\|=\min$, Fock $^{7)}$ derived in 1928 the condition $\left\langle n ; t\left|\frac{d}{d t}\right| n ; t\right\rangle=0$ which according to (2.41) - leads to the phase choice of unity for this extra phase factor. Though Fock did not consider cyclic time change as in (2.7) or (2.27), (2.34) his phase choice was universally accepted, and the extra phase factor was ignored for half a century. In the following we shall first show how $\gamma_{n}$ can be eliminated and then discuss that it can in general not be eliminated for cyclic processes.

From the definition (2.40) it follows that under a gauge tranformation (2.12) the Berry vector pctential transforms like:

$$
\begin{aligned}
\mathbf{A}^{n}(R) \rightarrow \mathbf{A}^{\prime n}(R) & =i\left\langle n ;\left.R\right|^{\prime}\left(\nabla|n, R\rangle^{\prime}\right)\right. \\
& =i\langle n, R| e^{-i \zeta_{n}(R)}\left(\nabla e^{i \zeta_{n}(R)}|n ; R\rangle\right) \\
& =i\langle n ; R|\nabla| n ; R\rangle+i e^{-i \zeta_{n}(R)}\left(\nabla e^{i \zeta_{n}(R)}\right)
\end{aligned}
$$

or

$$
\mathbf{A}^{n}(R) \longrightarrow \mathbf{A}^{\prime n}(R)=\mathbf{A}^{n}(R)-\nabla \zeta_{n}(R)
$$

Thus

$$
\gamma_{n}(t)=\int_{R(0)}^{R(t)} \mathbf{A}^{n}(R) d \mathbf{R} \rightarrow \int_{R(0)}^{R(t)} \mathbf{A}^{\prime n}(R) d \mathbf{R}=\gamma_{n}(t)-\zeta_{n}(R(t))+\zeta_{n}(R(0))=\gamma_{n}^{\prime}(t)
$$

We shall now do the calculations that led to (2.43) using the $|n ; R\rangle^{\prime}$ in place of the $|n ; R\rangle$. Then we obtain (2.43) with the primed quantities on the right hand side. Then using (2.12) we obtain for the primed quantities:

$$
e^{i \gamma_{n}^{\prime}(t)}|n, R(t)\rangle^{\prime}=e^{i \gamma_{n}^{\prime}(t)} e^{i \zeta_{n}(R(t))}|n ; R(t)\rangle .
$$

If $\zeta_{n}(R(t))$ is an arbitrary single-valued function modulo $2 \pi$ we can choose it such that the phase factor $e^{i \gamma_{n}^{\prime}(t)} e^{i \zeta_{n}(R(t))}$ becomes unity and we obtain in place of $(2.43)$ :

$$
\psi(t)=e^{-i \int_{0}^{t} E_{n}\left(t^{\prime}\right) d t^{\prime}}|n, R(t)\rangle .
$$

This also fulfills the initial condition (2.21). Since $|n, R\rangle^{\prime}$ is as valid a basis system (fulfilling (2.4); as is $|n, R\rangle$, we can use it and thus describe the time-developement of the state vector by (2.46) with the dynamical phase factor only. This is Fock's result. ${ }^{7)}$

The above arguments made use of the fact that $\zeta_{n}(R(t))$ was arbitrary. If after some period $T$ the environmental parameters return to their original value as for the closed path $\mathbf{C}(2.7)$, then one cannot choose $\zeta_{n}(R(T))$ freely to remove $\gamma_{n}(T)$.

Since $e^{i \zeta_{n}(R)}$ is a single-valued function of $R$ we must have for $R(T)=R(0)$

$$
e^{i \zeta_{n}(R(T))}=e^{i \zeta_{n}(R(0))} \quad \text { or } \quad \zeta_{n}(R(T))=\zeta_{n}(R(0))+2 \pi \cdot \text { integer } .
$$


Therefore according to $(2.45)$

$$
\begin{aligned}
\gamma_{n}(T) \rightarrow \gamma_{n}^{\prime}(T) & \equiv \oint_{\mathbf{C}} \mathbf{A}^{\prime n}(R) d \mathbf{R}=\gamma_{n}(T)-2 \pi \cdot \text { integer } \\
& =\oint_{\mathbf{C}} \mathbf{A}^{n}(R) d \mathbf{R}-2 \pi \cdot \text { integer }
\end{aligned}
$$

Thus $\gamma_{n}(T)$ - which is only defined modulo $2 \pi$ - is an invariant of the gauge transformation (2.12) and can therefore not be removed. We therefore have

$$
\psi(T)=e^{-i \int_{0}^{T} d t E_{n}(t)+i \gamma_{n}(T)}|n ; R(T)\rangle
$$

with the $\gamma_{n}(T)$ given by the loop integral over the closed path $\mathrm{C}$ of $(2.7)$ :

$$
\gamma_{n}(\mathbf{C}) \equiv \gamma_{n}(T)=\oint_{\mathbf{C}} i\left\langle r_{i} ; R\left|\frac{\partial}{\partial R^{i}}\right| n ; R\right\rangle d R^{i}=\oint_{\mathbf{C}} \mathbf{A}^{n}(R) d \mathbf{R} \text { modulo } 2 \pi .
$$

If we insert the initial condition (2.21) and (2.11) into (2.49) we obtain

$$
\psi(T)=e^{-i \int_{0}^{T} d t E_{n}(t)} e^{i \gamma_{n}(C)} \psi(0) .
$$

The phase angle $\gamma_{n}(T)$ is called the Berry phase angle, $e^{i \gamma_{n}(T)}$ is called the Berry phase factor.

We have shown that for a closed path the extra phase factor cannot be transformed away. This does not mean that $\gamma_{n}(C)$ could not be zero. Indeed this will turn out to be the case for many Hamiltonians $h(R)$. In this case the vector potential $A^{n}(R)$ need not be zero but will be trivial, which means given by

$$
\mathbf{A}^{n}(R)=\nabla \zeta(R)=-i e^{-i \zeta(R)} \nabla e^{i \zeta(R)},
$$

where $\zeta(R)$ is a well-defined function of $R$. Then, according to (2.44), it can be transformed to zero by the gauge transformation with $e^{-i \zeta(R)}$. The cases that we are interested in are those for which the Berry phase is different from zero. The Hamiltonian (2.1) is such a case, as we shall discuss in detail in the next section.

From (2.44) we see that the Berry vector potential transforms under the phase transformation (2.12) like the vector potential of electrodynamics transforms under a gauge transformation. The set of phase factors $e^{i \zeta_{n}(R)}$ form the group $U(1)$, they are continuous, differentiable, single-valued functions of the parameters $R$. We therefore have here a gauge theory with gauge group $U(1)$ and gauge potential $\mathbf{A}^{n}(R)$. This is the reason for which we call the phase transformation (2.12) a $U(1)$-gauge transformation. Whereas $\mathbf{A}^{n}(R)$ is not an invariant with respect to a gauge transformation but transforms like (2.44), the Berry phase is gauge invariant. Precisely, the phase factor $e^{i \gamma_{n}(T)}$ is $U(1)$ gauge invariant and the phase angle $\gamma_{n}(T)$ modulo $2 \pi$ is $U(1)$ gauge invariant (it is only defined modulo $2 \pi$ ). If the parameter space is three-dimensional and the parameter $R=\mathbf{R}$ is the three-dimensional coordinate vector then we have complete analogy with the electromagnetic theory. However, the physical meaning of these quantities is different, because the gauge potential (2.40) is defined in terms of the eigenvectors of the Hamiltonian and has nothing to do with electromagnetism. For 
an $f$-dimensional parameter space we have again a $U(1)$-gauge theory, but the gauge transformations and gauge potentials now depend upon an $f$-dimerisional parameter $R$ and the $A_{i}(R) ; i=1,2, \ldots f$, consist of $f$ components. It is called a $U(1)$ gauge theory over an $f$-dimensional base (= parameter) space.

In analogy to electrodynamics we can define a gauge field

$$
F_{i j}^{n}=\frac{\partial}{\partial R^{i}} A_{j}^{n}-\frac{\partial}{\partial R^{j}} A_{i}^{n} \quad i, j=1,2, \ldots f_{i}
$$

or (if $\mathbf{R}$ is a 3 -vector),

$$
\mathbf{F}^{n}=\nabla \wedge \mathbf{A}^{n}
$$

The corresponding 2 -form is:

$$
F^{n}=d A^{n}=\frac{\partial A_{j}^{n}}{\partial R^{i}} d R^{i} \wedge d R^{j}=\frac{1}{2} F_{i j}^{m} d R^{i} \wedge d R^{j} .
$$

This gauge field $F^{n}$ with $A^{n}$ given by (2.42) is also called Berry curvature. From (2.44) it follows immediately that the gauge field - like in every abelian gauge theory - is gauge invariant:

$$
F^{n} \rightarrow F^{\prime n}=\nabla \wedge \mathbf{A}^{n}=\mathbf{F}^{n}
$$

In formula (2.50) Stokes' theorem may be used to convert the line integral over the closed path $\mathbf{C}$ in the environments parameter space into a surface integal over any surface $S$ enclosed by the path $\mathbf{C}$. For 3-dimensional $\mathbf{R}$ this is:

$$
\gamma_{n}(T)=\oint_{\mathbf{C}} \mathbf{A}^{n}(R) d \mathbf{R}=\iint_{S} d \mathbf{S}\left(\nabla \wedge \mathbf{A}^{n}\right) \bmod 2 \pi
$$

and for an $f$-dimensional parameter space

$$
\gamma_{n}(T)=\oint_{\mathbf{C}} i\left\langle n ; R\left|\frac{\partial}{\partial R^{i}}\right| n ; R\right\rangle d R^{i}=\oint_{\mathbf{C}} A^{n}=\iint_{S} d A^{n}=\iint_{S} F^{n} \bmod 2 \pi .
$$

$S$ is the surface that subtends $\mathbf{C}$ and $d \mathbf{S}$ denotes the surface element in parameter space. The direction of $d \mathbf{S}$ is normal to the surface $S$ and the line element $d \mathbf{R}$ of $\mathbf{C}$ is traversed in the right-hand sense with respect to the normal.

Using (2.42) in $\left(2.55^{\prime}\right)$ we can express $\gamma_{n}(T)$ also in the form

$$
\gamma_{n}(T)=\iint_{S} F^{n}=\int d(i\langle n ; R|d| n ; R\rangle) \bmod 2 \pi
$$

or for the 3-dimensional case

$$
\gamma_{\boldsymbol{n}}(T)=\iint_{S} d \mathbf{S} \cdot \mathbf{F}=\iint d \mathbf{S} i \nabla \wedge(\langle n ; \mathbf{R}|\nabla| n ; \mathbf{R}\rangle)
$$


We have added mod $2 \pi$ in these equations because $\gamma_{n}(T)$ is defined only up to an integer times $2 \pi$. In these integrals the Berry curvature is:

$$
\begin{aligned}
F^{n} & =i(d\langle n ; R|) \wedge d|n ; R\rangle=-\operatorname{Im} d\langle n ; R|d| n ; R\rangle \\
& =-\operatorname{Im}\left(\frac{\partial}{\partial R^{i}}\langle n ; R|\right) \frac{\partial}{\partial R^{j}}|n, R\rangle d R^{i} \wedge d R^{j}
\end{aligned}
$$

or for the three-dimensional case

$$
\mathbf{F}^{n}=-\operatorname{Im}(\nabla\langle n ; \mathbf{R}|) \wedge \nabla|n ; \mathbf{R}\rangle .
$$

Equation $\left(2.56^{\prime}\right)$ shows that the Berry phase $\gamma_{n}(T)$ is analogous to the magnetic flux of the electromagnetic theory.

To see how non-trivial phases arise when two energy levels become degenerate for some values of the parameter, we express the Berry curvature in terms of the eigenvalues $E_{n}(R)$. We continue the calculation of $F$ in (2.57). Inserting the complete basis system

$$
1=\sum_{m}|m ; R\rangle\langle m ; R|
$$

we obtain

$$
\begin{aligned}
& F^{n}=-\operatorname{Im} \sum_{m}(\nabla\langle n ; R|)|m ; R\rangle \wedge\langle m ; R|\nabla| n ; R\rangle \\
& =-\operatorname{Im}\langle\overline{(n ; R|\nabla| n ; R}\rangle \wedge\langle n ; R|\nabla| n ; R\rangle \\
& -\operatorname{Im} \sum_{m \neq n}\langle\overline{m ; R|\nabla| n, R\rangle} \wedge\langle m ; R|\nabla| n ; R\rangle \text {. }
\end{aligned}
$$

The first term is zero because $\langle n ; R|\nabla| n ; R\rangle$ is purely imaginary. In the second term we use

$$
\langle m ; R|\nabla| n ; R\rangle=\langle m ; R|(\nabla h(R))| n, R\rangle \frac{1}{E_{n}(R)-E_{m}(R)} \text { for } n \neq m
$$

which can be easily proven (problem 2.2). * Then we obtain

$$
\mathbf{F}^{n}=-\operatorname{Im} \sum_{m \neq n} \frac{\langle n ; R|[\nabla h(R)]| m, R\rangle \wedge\langle m ; R|[\nabla h(R)]| n ; R\rangle}{\left[E_{n}(R)-E_{m}(R)\right]^{2}}
$$

and the Berry phase angle is given in tems of this quantity:

$$
\gamma_{n}(\mathbf{C})=\iint_{S} d \mathbf{S ~ F}^{n}(\operatorname{modulo} 2 \pi)
$$

where $S$ is any surface in the parameter space which subtends the closed curve $\mathbf{C}$ (we write this also as $\mathbf{C}=\partial S$ and call $\partial S$ the boundary of $S$ ).

The form (2.60) does not contain any phase factor of the basis vectors $|m ; R\rangle$. Whereas (2.40) is only defined for single-valued $|n, R\rangle$ and can therefore be defined only

* Problem 2.2. Show that for the eigenvectors $|n ; \mathbf{R}\rangle$ defined by (2.4) the relation (2.59) holds. 
on a patch where single-valued $|n ; R\rangle$ exist, the formula (2.60) can also be used for an area where single-valued $|n ; R\rangle$ do not exist.

The formula (2.60) also shows that the singularities of $F^{n}$ occur at those values of $R=R_{0}$ where the eigenvalues are degenerate $E_{n}\left(R_{0}\right)=E_{m}\left(R_{0}\right)$.

We conclude this part with the following remark: The Berry phase (2.50) has been calculated with single-valued basis vectors $|n ; R\rangle$ fulfilling (2.11). If we use instead vectors fulfilling $(2.11 \mathrm{a})$ (which may have their origin in non-single-valued phase transformations $e^{i \zeta_{n}(R(T))}=e^{i \zeta_{n}} e^{i \zeta_{n}(R(0))}$ :

$$
\left.|n ; \widetilde{R(T)}\rangle=e^{i \zeta_{n}(R(T))}|n ; R(T)\rangle=e^{i \zeta_{n}}|n ; R(0)\rangle\right)
$$

then there will be again an extra phase factor for $\psi(T)$ which is now given by

$$
\psi(T)=e^{-i \oint_{0}^{T} d t^{\prime} E_{n}\left(t^{\prime}\right)} e^{i \bar{\gamma}_{n}(C)} e^{i \zeta_{n}} \psi(0)
$$

where $\tilde{\gamma}_{n}(T)$ is the phase calculated from $(2.50)$ with the $|n ; R\rangle$ replaced by the $|\widetilde{n ; R}\rangle$. This phase angle; calculated with the non-single-valued basis vectors is not the Berry phase angle; the Berry phase angle is now given by $\tilde{\gamma}_{n}+\zeta_{n}$. The Berry phase is thus only defined by $(2.50)$ if the basis vectors are single valued.

From the above formulas we see that $\gamma_{n}(\mathbf{C})$ is independent of how the closed loop $\mathbf{C}$ is traversed (provided it is such that all our conditions are fufilled, which means for the case under consideration, that the loop is traversed slowly enough for the adiabatic approximation (2.24) to hold). The Berry phase angle $\gamma_{n}(T)$ thus does not depend upon the dynamics of the quantum system or upon the details of the environmental changes. It only depends upon the path $\mathbf{C}$ (with the provision of adiabaticity) and is thus a quantity determined by the geometry of the parameter space. It is therefore called the adiabatic geometric phase. Later we will also consider non-adiabatic cyclic evolutions and shall reserve the name geometric phase for the non-adiabatic generalization of the Berry phase $\gamma_{n}(\mathbf{C})$. 


\section{References}

1. All equation numbers of this kind refer to A. Bohm, Quantum Mechanics: Foundations and Applications, Second Edition, Springer-Verlag, New York, 1986, The roman number gives the chapter, the other number the equation.

2. A. Bohm, ibid, Chapter XII.

3. See e.g. C. Itzykson, J.B. Zuber, Quantum Field Theory, Section 4-1-4, McGraw-Hill publishers (1980).

4. M. Born and V. Fock, Z. Phys. 51, 165 (1928).

5. See reference 2 and reference 6 of section 1.

6. C.J. Isham, Modern Differential Geometry for Physicists, World Scientific Publishers (1989).

7. Reference 1 , section 1 . 


\section{A Spinning Quantum System in an External Magnetic Field}

\subsection{The Parameterization of the Basis Vectors}

In this lecture we will discuss in detail a quantum particle with magnetic moment $\mathbf{m}=\mu_{B} g \mathbf{J}$ in an external magnetic field, $\mathbf{B}(t)=B \mathbf{R}(t)$ whose direction $\mathbf{R}(t)$ is changing periodically. In particular we will consider the case in which the magnetic field direction precesses around the 3-direction. If the direction rotates slowly ("adiabatically") this system provides an application of the general ideas presented in section 2 . The Schrödinger equation for a magnetic moment in a precessing magnetic field has been solved exactly. ${ }^{1)}$ Therefore we need not restrict ourselves in this example to the adiabatic approximation and will obtain the non-adiabatic (Aharonov-Anandan) geometric phase. ${ }^{2)}$ With the help of this example we will then introduce in section 3.4 the non-adiabatic geometric phase for a general cyclic evolution. ${ }^{3)}$

The Hamiltonian is given according to $(2.1)$ by:

$$
h(\hat{\mathbf{R}}(t))=-B g \frac{e}{2 m c} \hat{\mathbf{R}}(t) \cdot \mathbf{J}=b \hat{\mathbf{R}}(t) \cdot \mathbf{J}
$$

where $\mathbf{J}$ is the angular momentum operator of the quantum physical system, $\hat{\mathbf{R}}(t)$ is the parameter that describes the changing environment, and $b=-B g \frac{e}{2 m c}$ is a constant. $\left(\frac{e \hbar}{2 m c}=\mu_{B}\right.$ is the Bohr magneton and $g$ is the Lande factor of the quantum particle. $b$ is a frequency and $b /(-g) \equiv \omega_{L}$ is called the Larmor frequency).

The parameter space of the environment (the set of all $\mathbf{R}$ ) is the unit sphere in two-dimensions, $\mathcal{S}^{2}$. We parameterize $\mathcal{S}^{2}$ by the polar angles $(\theta, \varphi)$ according to

$$
\hat{\mathbf{R}}=\hat{\mathbf{R}}(\theta, \varphi)=\left(\begin{array}{cc}
\sin \theta \cos \varphi \\
\sin \theta \sin \varphi \\
\cos \theta
\end{array}\right), \quad 0 \leq \theta \leq \pi .
$$

This parameterization associates unique values of the pair $(\theta, \varphi)$ to each unit vector $\hat{\mathbf{R}}$ except for the unit vector

$$
\mathbf{e}_{3} \equiv\left(\begin{array}{l}
0 \\
0 \\
1
\end{array}\right)
$$

of the north pole and the unit vector $-\mathbf{e}_{3}$ of the south pole. $\mathbf{e}_{3}$ and $-\mathbf{e}_{3}$ are given, respectively, by $\theta=0$ and $\theta=\pi$ for all values of $\varphi$ in the range $0 \leq \varphi<2 \pi ;$ the value of $\varphi$ is not determined when $\hat{\mathbf{R}}= \pm \mathbf{e}_{3}$. 
The special case in which the magnetic field precesses uniformly about the 3-axis, is described by

$$
\mathbf{B}(t)=B(\sin \theta \cos \omega t, \sin \theta \sin \omega t, \cos \theta)=B \hat{R}(\theta, \omega t)
$$

with $B, \theta$ and $\omega=\varphi / t$ being constants.

The precession of the magnetic field is shown in Fig. 3.1. For $t=0$ the magnetic field lies in the 1-3 plane

$$
\mathbf{B}(0)=B(\sin \theta, 0, \cos \theta)=\left(B_{12}, 0, B_{3}\right)=B \mathbf{R}(\theta, 0)
$$

Figure 3.1 also shows the angles $(\theta, \varphi)$ and a new angle $\tilde{\theta}$ which we will define later.

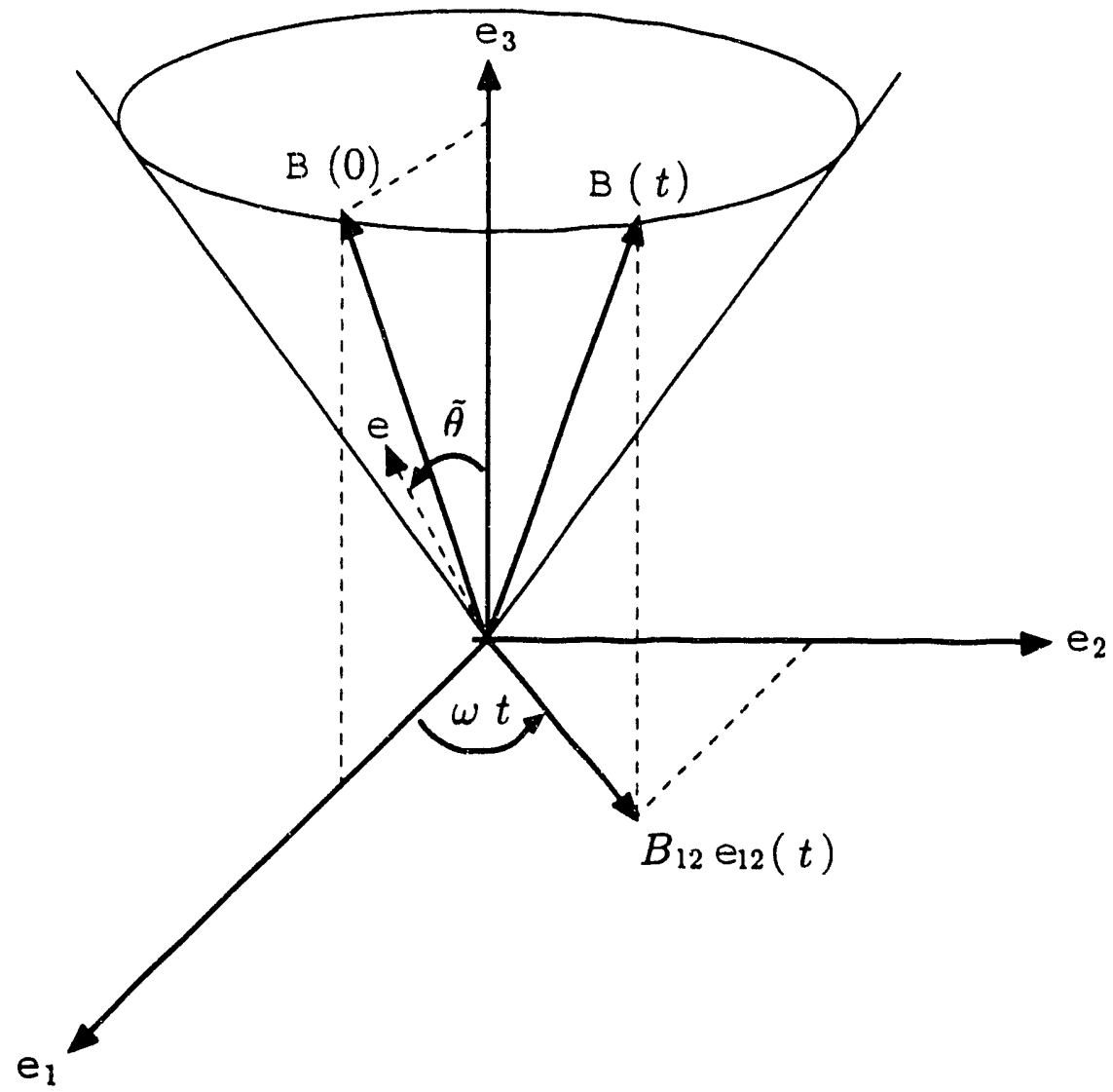

Figure 3.1. A quantal magnetic moment in an external magnetic field precesses uniformly around a cone of semiangle $\theta$ with $e_{3} .(\theta, \varphi)$ are the polar angles for the rotation of the external magnetic field. $(\tilde{\theta}, \varphi)$ are the "polar angles" for the evolution of the state of the magnetic moment.

The eigenvectors (2.4) for this example are given by

$$
h(R)|k ; R(\theta(t), \varphi(t))\rangle=b \hat{\mathbf{R}} \cdot \mathbf{J}|k ; R\rangle=b k|k ; R\rangle
$$


They are eigenvectors of the operator $\hat{\mathbf{R}}(t) \cdot \mathbf{J}$. The place of the energy quantum number $n$ in (2.4) is taken here by $k$ which is the quantum number for the component of angular momentum along the changing direction of the external magnetic field.

The eigenvalue $E_{k}(R(t))=b k$ of the time-dependent Hamiltonian $h(R(t))$ is for this example a constant, but the eigenvectors $|n ; R(t)\rangle$ and the eigenprojectors $\Lambda_{k}(R(t))=$ $|k ; R\rangle\langle k ; R|$ change in time. The eigenvalue $k$ is a constant but its physical interpretation changes in time, it is the value of the observable $\mathbf{R}(t) \cdot \mathbf{J}$ where the direction $\mathbf{R}(t)$ changes with respect to the laboratory frame (which is the observable given by the direction $e_{3}$ ).

If the vectors $|k ; R\rangle$ are also eigenvectors of $\mathbf{J}^{2}$, then the possible values of $k$ are given by

$$
k=-j, \quad \text { or }-j+1, \ldots, \quad \text { or }+j ; \quad \text { where } j=\text { integer or half integer }
$$

and $j(j+1)$ is the eigenvalue of the angular momentum $\mathbf{J}^{2}$. We assume here that $j$ and any other additional quantum numbers, which may be connected with the eigenvalues of the rotationally invariant part $H_{0}$ of the total Hamiltonian $H^{\text {total }}=H_{0}+h(\hat{\mathbf{R}})$, have fixed values and we suppress them throughout the current discussion as labels of the vectors $|k ; R\rangle$.

But it may also be that the $|k ; \hat{\mathbf{R}}\rangle$ are not eigenvectors of $\mathbf{J}^{2}$ and thus do not have a definite value of $j$ associated to them. This kind of vectors will be needed in the example of a diatomic molecule where $k$ is the eigenvalue of a quantum mechanical observable $\mathbf{X}$. J. The quantum mechanical observable $\mathbf{X}$ is the position operator (direction) of the internuclear axis of the molecule, which rotates in space. This observable describes the slowly changing quantal environment for the electron. The electron is the fast quantum system that follows instantaneously the motion of the molecule as a whole. The electronic angular momentum is not a good quantum number, because one does not have spherical symmetry but only axial symmetry about the internuclear axis $\mathbf{X}$. So the total Hamiltonian commutes with $\mathbf{X} \cdot \mathbf{J}$ but not with $\mathbf{J}^{2}$ ( $\mathbf{J}$ being in this case the electronic angular momentum or electronic spin) and the molecular states are not $\mathbf{J}^{2}$ eigenstates.

In this case the possible values, that $k$ can take, are not given by (3.6) and $2 k$ need not have a priori an integer value. We shall, however, see below that also in this case one can prove that $k$ must be an integer or half integer. Thus $k$ can be interpreted as the component of angular momentum along the changing internuclear axis.

The vectors of (3.5) are parameterized by the unit vector $\hat{\mathbf{R}}$, or by the polar angles $(\theta, \varphi)$. They can be obtained by applying $(\theta, \varphi)$-dependent rotations to an eigenvector $|k ; \dot{\epsilon}\rangle$ of the component of angular momentum in the direction of the north pole, $J_{3}=$ $e_{3} \cdot s$.

There are many $(\theta, \varphi)$-dependent rotations $\mathcal{R}(\theta, \varphi) \in \mathrm{SO}(3)$ which when applied to $\mathbf{e}_{3}$ give the arbitrary unit vector $\hat{\mathbf{R}}(\theta, \varphi)$. We choose the product of rotation*

$$
\begin{aligned}
\mathcal{R}(\theta, \varphi) \mathbf{e}_{3} & =\mathcal{R}_{3}(\varphi) \mathcal{R}_{2}(\theta) \mathcal{R}_{3}(-\varphi) \mathbf{e}_{3}=\mathcal{R}_{3}(\varphi) \mathcal{R}_{2}(\theta) \mathbf{e}_{3}=\mathcal{R}_{3}(\varphi) \hat{\mathbf{R}}(\theta, 0) \\
& =\hat{\mathbf{R}}(\theta, \varphi)
\end{aligned}
$$

* Problem 3.1. Show that the rotation $\mathcal{R}(\theta, \varphi)$ defined by (3.7) transforms the vector $e_{3}$ represented by (3.3) into the vector $\hat{\mathbf{R}}(\theta, \varphi)$ represented by (3.2). Use the standard representation matrices 
where $\mathcal{R}_{3}(-\varphi)$ does nothing to the unit vector $\mathbf{e}_{3}$ and where $\mathcal{R}_{2}(\theta)$ produces the unit vector $\hat{\mathbf{R}}(\theta, 0)$ which lies in the $1-3$ plane at an angle $\theta$ with respect to the $\mathbf{e}_{3}$-axis. The rotation $\mathcal{R}_{3}(-\varphi)$ has been included in the definition of $\mathcal{R}(\theta, \varphi)$ in order for the rotation $\mathcal{R}(0, \varphi)$ to be independent of $\varphi$.

Rotations, like any other continuous transformations, are represented in the space of quantum physical states by unitary operators (representing the group $\mathrm{SU}(2)$ ). The unitary operators that represent the rotations $\mathcal{R}_{3}(\varphi)$ and $\mathcal{R}_{2}(\theta)$ are given by

$$
\begin{aligned}
U_{3}(\varphi) & =e^{-i \varphi J_{3}} \equiv I+\frac{\varphi}{i} J_{3}+\frac{1}{2 !}\left(\frac{\varphi}{i} J_{3}\right)^{2}+\cdots, \\
U_{2}(\theta) & =e^{-i \theta J_{3}}
\end{aligned}
$$

and an analogous expression holds for rotations about the $\mathbf{e}_{1}$-axis. The product of two or more rotations is represented by the product of the corresponding operators. The rotation $\mathcal{R}(\theta, \varphi)$ of $(3.7)$ is thus represented by the operator

$$
U(\theta, \varphi)=U_{3}(\varphi) U_{2}(\theta) U_{3}(-\varphi)=e^{-i \varphi J_{3}} e^{-i \theta J_{2}} e^{i \varphi J_{3}}
$$

We now choose a fixed normalized eigenvector $\left|k ; \mathbf{e}_{3}\right\rangle$ of $\mathbf{e}_{3} \cdot \mathbf{J}=\hat{\mathbf{R}}(0,0) \cdot \mathbf{J}$ and apply to it the unitary operator $U(\theta, \varphi)$. The resulting vector

$$
|k ; \theta \varphi\rangle \equiv U(\theta, \varphi)\left|k ; \hat{\mathbf{e}}_{3}\right\rangle=e^{-i \varphi J_{3}} e^{-i \theta J_{2}} e^{i \varphi J_{3}}\left|k ; \hat{\mathbf{e}}_{3}\right\rangle,
$$

is an eigenvector of the operator $\hat{\mathbf{R}}(\theta, \varphi) \cdot \mathbf{J}$ with eigenvalue $k$ :

$$
\hat{\mathbf{R}}(\theta, \varphi) \cdot \mathbf{J}|k ; \theta, \varphi\rangle=k|k ; \theta, \varphi\rangle .
$$

To prove (3.12) one has to use the following transformation property of the angular momentum operators $J_{i}$ which follow from their commutation relations: ${ }^{4)}$

$$
\begin{aligned}
e^{-i \theta J_{2}} J_{3} e^{i \theta J_{2}} & =J_{3} \cos \theta+J_{1} \sin \theta, \\
e^{-i \varphi J_{3}} J_{1} e^{i \varphi J_{3}} & =J_{1} \cos \varphi+J_{2} \sin \varphi . \\
e^{-i \varphi J_{3}} J_{2} e^{i \varphi J_{3}} & =J_{1}(-\sin \varphi)+J_{2} \cos \varphi
\end{aligned}
$$

for $\mathcal{R}_{3}(\varphi)$ and $\mathcal{R}_{2}(\theta)$ :

$$
\mathcal{R}_{3}(\varphi)=\left(\begin{array}{ccc}
\cos \varphi & -\sin \varphi & 0 \\
\sin \varphi & \cos \varphi & 0 \\
0 & 0 & 1
\end{array}\right) \quad, \quad \mathcal{R}_{2}(\theta)=\left(\begin{array}{ccc}
\cos \theta & 0 & \sin \theta \\
0 & 1 & 0 \\
-\sin \theta & 0 & \cos \theta
\end{array}\right)
$$

(Note the change in sign for the angle $\theta$ ).

Problem 3.2. Find a rotation $\mathcal{R}^{\prime}(\theta, \varphi)$ which has the following properties

i. It transforms $\mathbf{e}_{3}$ into the vector $\hat{\mathbf{R}}(\theta, \varphi)$.

ii. The rotation $\mathcal{R}^{\prime}(\theta=\pi, \varphi)$ is independent of $\varphi$. 
The vector (3.11) is a (continuous, differentiable) vector-valned function of $(\theta, \varphi)$. This vector-valued function gives a unique vector for all $\hat{\mathbf{R}}$ except for the south pole $\hat{\mathbf{R}}=-\mathbf{e}_{3}$. At the south pole, $\theta=\pi,(3.13)$ becomes

which implies

$$
e^{-i \pi J_{2}} J_{3} e^{i \pi J_{2}}=-J_{3}
$$

$$
e^{-i \pi J_{2}} e^{-i \varphi J_{3}} e^{i \pi J_{2}}=e^{i \varphi J_{3}}
$$

and may be used to obtain

$$
\begin{aligned}
|k ; \pi \varphi\rangle & =e^{-i \varphi J_{3}} e^{-i \pi J_{2}} e^{i \varphi J_{3}}\left|k ; \hat{\mathbf{e}}_{3}\right\rangle \\
& =e^{-i \pi J_{2}} e^{2 i \varphi J_{3}}\left|k ; \hat{\mathbf{e}}_{3}\right\rangle \\
& =e^{-i \pi J_{2}} e^{2 i k \varphi}\left|k ; \hat{\mathbf{e}}_{3}\right\rangle .
\end{aligned}
$$

This shows that at the south pole different vectors of the unit ray are obtained as $\varphi$ varies in the range $0 \leq \varphi<2 \pi$. $\varphi$ also varies in the range $0 \leq \varphi<2 \pi$ at the north pole $\mathbf{e}_{3}=\hat{\mathbf{R}}(0, \varphi)$ but $|k ; \theta, \varphi\rangle$ is single-valued at the north pole because $|k ; 0 \varphi\rangle$ does not depend upon $\varphi$ as a result of the inclusion of the rotation $\mathcal{R}_{3}(-\varphi)$ in the definition of $\mathcal{R}(\theta, \varphi) .|k ; \theta, \varphi\rangle$ is thus a single-valued vector function everywhere on $\mathcal{S}^{2}$ except on the south pole.

A vector-valued function which is well-defined at the south pole but not at the north pole is obtained by a gauge transformation (2.12):

$$
|k ; \theta, \varphi\rangle^{\prime}=e^{-i 2 k \varphi}|k ; \theta, \varphi\rangle=e^{-i \varphi J_{3}} e^{-i \theta J_{2}} e^{-i \varphi J_{3}}|k ; 0,0\rangle .
$$

The new vector $|k ; \theta, \varphi\rangle^{\prime}$ differs from $|k ; \theta, \varphi\rangle$ by the gauge transformation $e^{i \zeta(\theta, \varphi)}=$ $e^{-i 2 k \varphi}$. At the south pole $|k ; \theta, \varphi\rangle^{\prime}$ evaluates to a single vector

$$
|k ; \pi, \varphi\rangle^{\prime}=e^{-i \pi J_{2}}\left|k ; \hat{\mathbf{e}}_{3}\right\rangle
$$

but at the north pole it evaluates to many vectors

$$
|k ; 0, \varphi\rangle^{\prime}=e^{-2 i k \varphi}\left|k ; \hat{\mathbf{e}}_{3}\right\rangle
$$

It therefore can be used everywhere on $\mathcal{S}^{2}$ except on the north pole. Either vector (3.11) or (3.19) can be used in the overlap region of the two open patches which consists of $\mathcal{S}^{2}$ with both poles excluded.

The vector $|k ; \pi, \varphi\rangle^{\prime}$ is an eigenvector of $J_{3}$ with eigenvalue $-k$ and an eigenvector of $\hat{\mathbf{R}}(\pi, \varphi) \cdot \mathbf{J}=-\mathbf{e}_{\mathbf{3}} \cdot \mathbf{J}=-J_{\mathbf{3}}$ with eigenvalue $k$ (Problem 3.3).

We thus see that two different para" and $|k ; \theta, \varphi\rangle^{\prime}$ differ only by the phase factor $e^{-i 2 k \varphi}$ the projection operators and subspaces are, however, the same:

$$
|k ; \theta, \varphi\rangle\langle k ; \theta, \varphi|=| k ; \theta, \varphi\rangle^{\prime}\langle k ; \theta, \varphi|
$$

* Problem 3.9. Show that the vector (3.20) is an eigenvector of $J_{3}$ with eigenvalue $(-k)$ and an eigenvector of $h(R(\theta=\pi, \varphi))$ fulfilling (3.5). 


\subsection{Berry Connection and Berry Phase for Adiabatic Evolutions - Magnetic Monopole Potentials}

We now calculate the gauge potential (Mead-Berry connection) for the adiabatic evolution of the Hamiltonian (3.1). The easiest is to calculate it as the one-form

$$
A^{k^{\prime} k}\left(R^{i}\right)=A_{i}^{k^{\prime} k} d R^{i}=i\left\langle k^{\prime} ; R^{j}\left|\frac{\partial}{\partial R^{i}}\right| k ; R^{j}\right\rangle d R^{i} .
$$

As the vectors (3.11) and (3.19) are parameterized by the angles $\theta, \varphi$ we will use for $R^{i}$ the polar coordinates: $\mathbf{R}=(r \sin \theta \cos \varphi, r \sin \theta \sin \varphi, r \cos \theta)$.

We are mainly interested in the diagonal matrix elements $A^{k k} \equiv A^{k}$ but include in our derivation also the off-diagonal matrix elements without much additional effort. vector

If $d R^{i}$ are the cartesian components, then $A_{i}^{k^{\prime} k}$ are the cartesian components of the

$$
\begin{aligned}
\mathbf{A}^{k^{\prime} k}(\theta, \varphi) & =i\left\langle k^{\prime} ; \theta \varphi\left|\nabla_{R}\right| k ; \theta \varphi\right\rangle \\
& =\left\langle k^{\prime} ; \hat{\mathbf{e}}_{3}\left|i U^{\dagger}(\theta, \varphi)\left(\hat{\mathbf{e}}_{r} \frac{\partial}{\partial r}+\hat{\mathbf{e}}_{\theta} \frac{1}{r} \frac{\partial}{\partial \theta}+\hat{\mathbf{e}}_{\varphi} \frac{1}{\sin \theta \partial \varphi}\right) U(\theta, \varphi)\right| k ; \hat{\mathbf{e}}_{3}\right\rangle \\
& =\hat{\mathbf{e}}_{r} \hat{A}_{r}^{k^{\prime} k}(\theta, \varphi)+\hat{\mathbf{e}}_{\theta} \hat{A}_{\theta}^{k^{\prime} k}(\theta, \varphi)+\hat{\mathbf{e}}_{\varphi} \hat{A}_{\varphi}^{k^{\prime} k}(\theta, \varphi) .
\end{aligned}
$$

$\hat{\mathbf{e}}_{r}, \hat{\mathbf{e}}_{\theta}, \hat{\mathbf{e}}_{\varphi}$ are the unit vectors along the $r-, \theta$ - and $\varphi$-direction respectively and the standard form for the vector $\nabla$ has been used. $\hat{A}_{r}, \hat{A}_{\theta}, \hat{A}_{\varphi}$ are the spherical orthonormal components of $\mathbf{A}$. As $|k ; \theta, \varphi\rangle=U(\theta, \varphi)\left|k ; \hat{e}_{3}\right\rangle$ is independent or $r$

$$
\hat{A}_{r}^{k^{\prime}, k}=0
$$

The connection 1-form therefore is

$$
\begin{aligned}
A^{k^{\prime} k}(\theta, \varphi) & =\hat{A}_{\theta} r d \theta+\hat{A}_{\varphi} r \sin \theta d \varphi \\
& =i\left\langle k^{\prime} ; \theta, \varphi\left|\frac{\partial}{\partial \theta}\right| k ; \theta, \varphi\right\rangle d \theta+i\left\langle k^{\prime} ; \theta, \varphi\left|\frac{\partial}{\partial \varphi}\right| k ; \theta, \varphi\right\rangle d \varphi \\
& \equiv A_{\theta}^{k^{\prime} k} d \theta+A_{\varphi}^{k^{\prime} k} d \varphi
\end{aligned}
$$

where $A_{\theta}^{k^{\prime} k}, A_{\varphi}^{k^{\prime} k}$ are the spherical covariant components of $\mathbf{A}$.

It is easiest to calculate the covariant components and then - if one wants to obtain the spherical orthonormal components from comparison of the first and third row of (3.26).

We first use the vectors (3.11). In this gauge we obtain

$$
A_{\theta}^{k^{\prime} k}=i\left\langle k^{\prime} ; \theta, \varphi\left|\frac{\partial}{\partial \theta}\right| k ; \theta, \varphi\right\rangle=\left\langle k^{\prime} \hat{\mathbf{e}}_{3}\left|i U^{\dagger}(\theta, \varphi) \frac{\partial}{\partial \theta} U(\theta, \varphi)\right| k ; \hat{\mathbf{e}}_{3}\right\rangle=r \hat{A}_{\theta}
$$

and

$$
A_{\varphi}^{k^{\prime} k}=i\left\langle k^{\prime} \theta, \varphi\left|\frac{\partial}{\partial \varphi}\right| k ; \theta, \varphi\right\rangle=\left\langle k^{\prime} ; \hat{\mathbf{e}}_{3}\left|i U^{\dagger}(\theta, \varphi) \frac{\partial}{\partial \varphi} U(\theta, \varphi)\right| k ; \hat{\mathbf{e}}_{3}\right\rangle=r \sin \theta \hat{A}_{\varphi}
$$


We calculare:

$$
\begin{aligned}
i U^{\dagger}(\theta, \varphi) \frac{\partial U(\theta, \varphi)}{\partial \theta} & =i e^{-i \varphi J_{3}} e^{i \theta J_{2}} e^{i \varphi J_{3}} \frac{\partial}{\partial \theta} e^{-i \varphi J_{3}} e^{-i \theta J_{2}} e^{i \varphi J_{3}} \\
& =e^{-i \varphi J_{3}} J_{2} e^{i \varphi J_{3}} \\
& =\left(J_{2} \cos \varphi-J_{1} \sin \varphi\right)
\end{aligned}
$$

and

$$
\begin{aligned}
i U^{\dagger}(\theta, \varphi) \frac{\partial U(\theta, \varphi)}{\partial \varphi} & =\left[U^{\dagger}(\theta, \varphi) J_{3} U(\theta, \varphi)-U^{\dagger}(\theta, \varphi) U(\theta, \varphi) J_{3}\right] \\
& =\left[-\left(J_{1} \cos \varphi+J_{2} \sin \varphi\right) \sin \theta+J_{3}(\cos \theta-1)\right]
\end{aligned}
$$

Inserting this into (3.27), (3.28) we obtain:

$$
\begin{aligned}
& A_{\theta}^{k^{\prime} k}(\theta, \varphi)=\left\langle k^{\prime} ; \hat{\mathbf{e}}_{3}\left|\left(J_{2} \cos \varphi-J_{1} \sin \varphi\right)\right| k ; \hat{\mathbf{e}}_{3}\right\rangle \\
& A_{\varphi}^{k}{ }^{\alpha}(\varphi, \varphi)=\left\langle k^{\prime} ; \hat{\mathbf{e}}_{3}\left|\left[-\left(J_{1} \cos \varphi+J_{2} \sin \varphi\right) \sin \theta+J_{3}(\cos \theta-1)\right]\right| k ; \hat{\mathbf{e}}_{3}\right\rangle .
\end{aligned}
$$

For the diagonal matrix elements, $J_{1}$ and $J_{2}$ do not c untribute so that we obtain

$$
\begin{aligned}
& A_{\theta}^{k}(\theta, \varphi)=0 \\
& A_{\varphi}^{k}(\theta, \varphi)=\left\langle k ; \hat{\mathbf{e}}_{3}\left|J_{3}(\cos \theta-1)\right| k ; \hat{\mathbf{e}}_{3}\right\rangle=-k(1-\cos \theta), \quad \theta \neq \pi .
\end{aligned}
$$

Thus the vector potential $\mathbf{A}$ has only a component in the $\varphi$-dirt ition which is given by:

$$
\hat{\mathbf{A}}^{k}(\theta, \varphi)=\hat{\mathbf{e}}_{\varphi} \frac{k(\cos \theta-1)}{r \sin \theta}, \quad \theta \neq \pi .
$$

We now calculate the connection using the vector-functions $|n ; \hat{\mathbf{R}}\rangle^{\prime}$ of $(3.19)$ which are single-valued everywhere except the north pole. We obtain in a similar way as above

$$
\begin{aligned}
& A_{\theta}^{k^{\prime} k}(\theta, \varphi)=\left\langle k^{\prime} ; \hat{\mathbf{e}}_{3}\left|\left(\nu_{2} \cos \varphi-J_{1} \sin \varphi\right)\right| k ; \hat{\mathbf{e}}_{3}\right\rangle^{\prime} \\
& A_{\varphi}^{k^{\prime} k}(\theta, \varphi)=\left\langle k^{\prime} ; \hat{\mathbf{e}}_{3}\left|\left[-\left(J_{1} \cos \varphi+J_{2} \sin \varphi\right) \sin \theta+J_{3}(\cos \theta-1)+2 k\right]\right| k ; \hat{\mathbf{e}}_{3}\right\rangle
\end{aligned}
$$

The diagonal matrix elements are:

$$
\begin{aligned}
& A_{\theta}^{\prime k}(\theta, \varphi)=0, \\
& A_{\varphi}^{\prime k}(\theta, \varphi)=\left\langle k ; \hat{\mathbf{e}}_{3}\left|\left[J_{3}(\cos \theta-1)+2 k\right]\right| k ; \hat{\mathbf{e}}_{3}\right\rangle^{\prime}=k(\cos \theta+1), \quad \theta \neq 0
\end{aligned}
$$

and the vector potential is given by:

$$
\mathbf{A}^{\prime k}(\theta, \varphi)=\hat{\mathbf{e}}_{\varphi} \frac{k(\cos \theta+1)}{r \sin \theta} \quad \theta \neq 0
$$

According to the general theory of section 2 we expect from (2.44) and $\zeta_{k}=-2 k \varphi$ :

$$
A^{\prime k}-A^{k}=-d \zeta_{k}(\theta, \varphi)=d(2 k \varphi)=2 k d \varphi
$$

or for the vectors

$$
\mathbf{A}^{\prime k} \cdots \mathbf{A}^{k}=-\nabla \zeta_{k}=2 k \frac{1}{r \sin \theta} \mathbf{e}_{\varphi}
$$


Comparing (3.39) with (3.34) we have indeed $A_{\varphi}^{\prime k}-A_{\varphi}^{k}=2 k$ in agreement with (3.41) and (3.42).

We now calculate the Berry curvature. According to $(2.53 \mathrm{c})$ :

$$
F^{k}=d A^{k}=\frac{\partial A_{\theta}^{k}}{\partial \varphi} d \varphi \wedge d \theta+\frac{\partial A_{\varphi}^{k}}{\partial \theta} d \theta \wedge d \varphi
$$

where we have already made use of (3.25). Using (3.33) and (3.34) we obtain

$$
F^{k}=-k \sin \theta d \theta \wedge d \varphi \equiv F_{\theta \varphi}^{k} d \theta \wedge d \varphi
$$

Thus except for the component

$$
F_{\theta \cdot \rho}^{k}=-F_{\varphi \theta}^{k}=-k \sin \theta
$$

all spherical covariant components of $F_{i j}$ are zero.

We now use (2.55) to calculate the Berry phase angle for a closed path $\mathbf{C}$ on the unit sphere $\mathcal{S}^{2}$ :

$$
\begin{aligned}
\gamma_{k}(\mathbf{C}) & =\oint_{\mathbf{C}} A^{k}=\oint_{\mathbf{C}} i\langle k ; \theta(t), \varphi(t)|d| k ; \theta(t), \varphi(t)\rangle \\
& =\int_{S} F^{k}=-k \int_{S} \sin \theta d \theta \wedge d \varphi=-k \int_{S} d \Omega \quad \bmod 2 \pi
\end{aligned}
$$

where $S$ is the surface (any surface) which spans the closed curve $\mathbf{C}$ (i.e. for which $\mathbf{C}$ is the boundary, $\mathbf{C}=\partial S$ ) and where $d \Omega$ is the solid angle element (area element of the unit sphere). We will denote by $\Omega(\mathbf{C})$ the solid angle that $\mathbf{C}$ subtends:

$$
\Omega(\mathbf{C})=\int_{S} \sin \theta d \theta \wedge d \varphi
$$

Then (3.46) can be written

$$
\gamma_{k}(\mathbf{C})=-k \Omega(\mathbf{C}) \quad \bmod 2 \pi .
$$

According to (3.46) $S$ is the surface that lies above $\mathbf{C}$ when one traverses the curve $\mathbf{C}$ (the direction in which $\mathbf{C}$ is traversed points along the fingers and the normal of $S$, i.e. the direction of $d \theta \wedge d \varphi$ points along the thumb of the right hand; cf. Fig. 3.2). Now let us consider the surface $S^{\prime}$ below $C$. Then again

$$
\gamma_{k}(\mathbf{C})=\int_{S_{\prime}} F^{k}=-k \int_{S_{\prime}} \sin \theta d \theta \wedge d \varphi=-k\left(-\int_{\mathcal{S}^{2} \backslash S} \sin \theta d \theta \wedge d \varphi\right)
$$

where the direction of the normal of $S^{\prime}$ and the direction in which $\mathbf{C}$ is traversed are again given by the right-hand rule. This means that the normal of $S^{\prime}$ points into the sphere $\mathcal{S}^{2},\left(\mathbf{C}=\partial S^{\prime}\right)$ and the normal of $S$ points out of the sphere $\mathcal{S}^{2},(\mathbf{C}=\partial S)$. In 
the equation (3.49) we denote by $\mathcal{S}^{2} \backslash S$ the surface with the same area as $S^{\prime}$ but whose normal points out of the sphere $\mathcal{S}^{2}$. We rewrite (3.4a) in the form:

$$
\gamma_{k}(\mathbf{C})=k\left(\int_{\mathcal{S}^{2}} \sin \theta d \theta \wedge d \varphi-\int_{S} \sin \theta d \theta \wedge d \varphi\right)
$$

As the integral of $d \Omega$ over the whole unit sphere is $4 \pi$ and the integral over $S$ is given by (3.47) we obtain:

$$
\gamma_{k}(\mathbf{C})=+k(4 \pi-\Omega(\mathbf{C})) \text { modulo } 2 \pi
$$

From this we conclude that $k$ is integer or half integer, because from comparing (3.50) and (3.48) it follows

$$
-k \Omega(\mathrm{C})=4 \pi k-k \Omega(\mathrm{C}) \bmod 2 \pi
$$

which can only be fulfilled if

$$
k=0, \pm \frac{1}{2} \pm 1, \pm \frac{3}{2}, \pm 2 \cdots .
$$

If the basis vectors (3.11) and (3.19) are also eigenvectors of $\mathrm{J}^{2}$ then the possible values of $k$ are already obtained from (3.6). But if these vectors are not $\mathbf{J}^{2}$-eigenvectors - as is the case for the molecule - then (3.52) is a new result. ${ }^{5}$ )

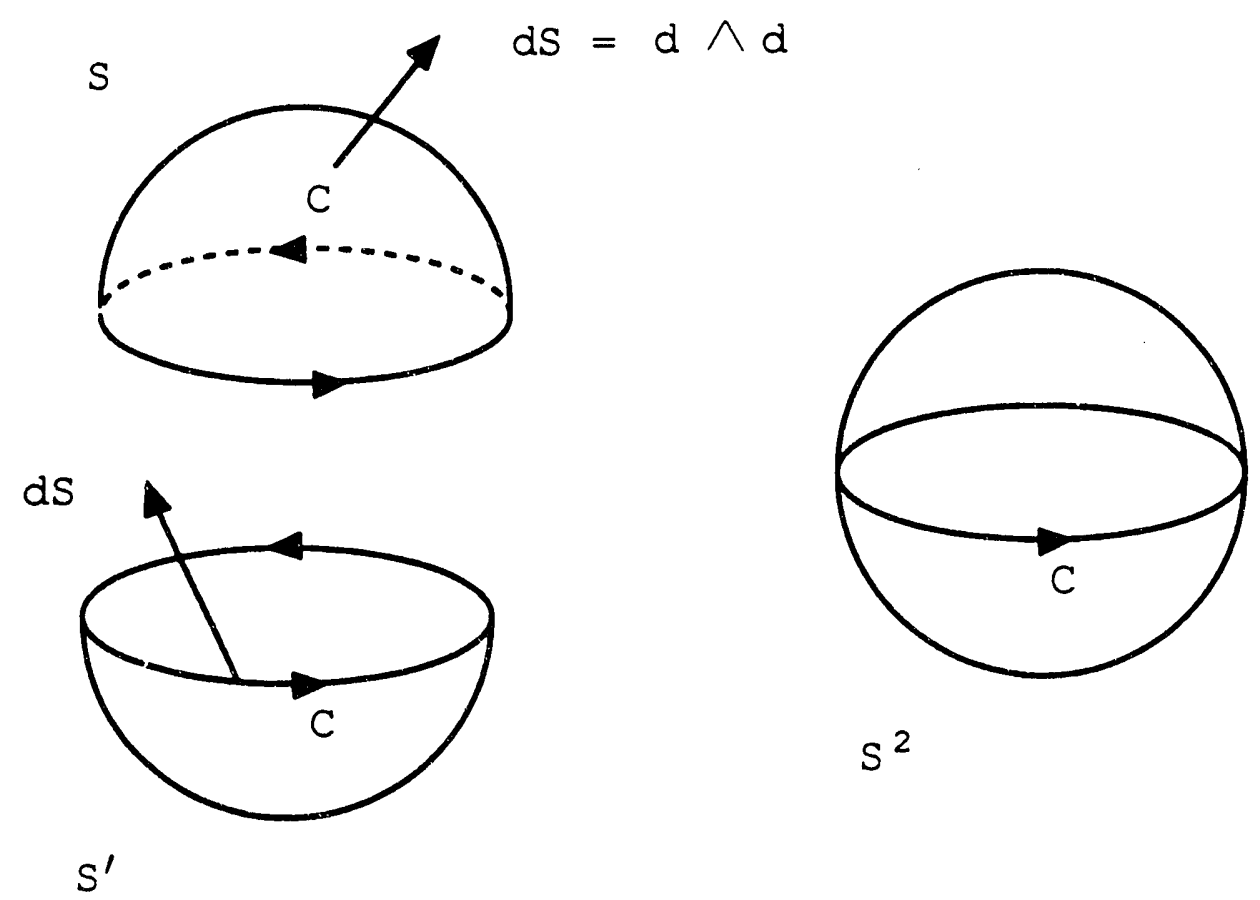

Figure 3.2. The difference of the line integrals of $A$ and $A^{\prime}$ can be transformed, using Stokes' theorem, into an integral over the closed 2-surface $S \cup S^{\prime}$. 
So far $\mathbf{C}$ could have been any closed path on the unit sphere. For the special path given by (3.4) and shown in Fig. 3.1.

$$
\mathrm{C}_{1}: R(\theta(t), \varphi(t))=R(\theta=\text { const., } \varphi=\omega t)
$$

the Berry phase (3.48) is

$$
\gamma_{k}\left(\mathbf{C}_{1}\right)=-k \int_{0}^{2 \pi} \int_{0}^{\theta} \sin \theta d \theta d \varphi=-k 2 \pi(1-\cos \theta) .
$$

where $\theta$ is the constant angle shown in Fig. 3.1. We shall use this expression below when we consider non-adiabatic cyclic evolution.

If $F_{\theta \varphi}=-F_{\varphi \theta}$ is the only non-zero spherical covariant component of $F$ then the only non-zero spherical orthonormal component is $\hat{F}_{\theta \varphi}=-\hat{F}_{\varphi \theta}$. The 2-form $F$ is given by

$$
F=F_{\theta \varphi} d \theta \wedge d \varphi=-k \sin \theta d \theta \wedge d \varphi=\hat{F}_{\theta \varphi}(r d \theta)(r \sin \theta d \varphi)=\vec{F} \cdot d \vec{S}
$$

and we immediately read off the value of $\hat{F}_{\theta \varphi}$ :

$$
\hat{F}_{\theta \varphi}=-\frac{k}{r^{2}} \quad \text { or } \quad \vec{F}=-\frac{k}{r^{2}} \hat{\mathbf{e}}_{r}=-\frac{k}{r^{2}} \hat{\mathbf{R}}(\theta, \varphi)
$$

where $\hat{\mathbf{e}}_{\mathbf{r}}$ is the unit vector in the radial direction which we had denoted by $\hat{\mathbf{R}}(\theta, \varphi)$.

The gauge field (3.55) has a very familiar form, it is the form of the magnetic field of a monopole. An imaginary electromagnetic system, with the vector potential given by (3.35) or (3.40) and the magnetic field given by (3.55), had been envisioned by Dirac more than 50 years ago ${ }^{6}$ and has intrigued several generations of theoreticians and experimentalists. ${ }^{7}$ It consists of a charge $e$ and a magnetic monopole with magnetic monopole strength $g$ with the vector $\hat{\mathbf{R}}$ pointing from $g$ to $e$. The magnetic field $\mathbf{B}^{\text {mon. }}$ of this charge-monopole system is given by $e \mathbf{B}^{\text {mon. }}=e \frac{g}{4 \pi} \frac{\hat{\mathbf{R}}}{r^{2}}$ and the electromagnetic vector potential $e \mathbf{A}^{\text {mon. }}$ is given by (3.35), (3.40)) with $k \rightarrow-\frac{e g}{4 \pi}$. For this magnetic monopole the field and the vector potential are supposed to be electromagnetic.

If one such magnetic monopole exists with magnetic charge $g$, then all electric charges must be multiples of $2 \pi / g$ because $k$ is integer or half integer according to (3.52). This would be a remarkable explanation of one of the basic facts in nature. Unfortunately, in spite of all efforts, such electromagnetic type of monopoles have never been found.

The field $\mathbf{F}$ of (3.55) and the vector potential $\mathbf{A}$ of (3.35) and (3.40) are not electromagnetic in nature. They have their origin in a quantum system spinning with fixed angular momentum component $k$ around the axis $\hat{\mathbf{R}}(t)$. The analogy between classical mechanical systems of this type and the charge-monopole system has been known for some time. ${ }^{8)}$ The Hamiltonian $h(R(t))$ of (3.1) also appears for quantum systems in a quantal environement, e.g. for diatomic molecules, because there is a magnetic field in the direction of the internuclear axis $\hat{\mathbf{R}}(t)$ which changes while the molecule as a whole rotates. As a consequence, the fast (electronic) motion about the body axis "induces" the gauge potential (3.35), where $k$ is the "fast" angular momentum along the internuclear axis. So the dynamics of the collective motion contains the monopole vector potential (3.35). (The canonical momenta: $P_{i}$ of the collective motion go into the 
gauge-covariant momenta $\left.P_{i} \rightarrow P_{i}-A_{i}\right)$. As a consequence, the effective Hamiltonian of the slow collective motion (the quantal environment) is governed - in addition to the radial potential - by the monopole vector potential. ${ }^{9)}$ But this is not an electromangetic vector potential because the electromagnetic constant $\frac{e g}{4 \pi}$ is replaced by the motion constant $k$. Thus there "is" a "motional monopole" in the molecule. But it cannot come out of it because the monopole is the consequence of the fast (electronic) motion which is the other part of the molecule, without which it cannot "be".

The Mead-Berry potential of the molecule can be derived directly from the Schrödinger equation of the molecule if one does not make the drastic assumption of the Born-Oppenheimer approximation. ${ }^{9)}$ The same arguments should hold for any other quantum physical system that can be visualized as a spinning bead which can slide along a rod that rotates slowly in space. 


\subsection{The Exact Solution of the Schrödinger Equation}

So far we have not discussed the time evolution of a state vector $\psi(t)$ for our example (3.1), i.e. the solutions of the Schrödinger equation

$$
i \frac{\partial \psi}{\partial t}=h(t) \psi(t) \quad ; \quad h(t)=b \hat{\mathbf{R}}(\theta, \omega t) \cdot \mathbf{J}
$$

In particular we are interested in solutions which describe cyclic evolutions (2.34):

$$
|\psi(T)\rangle\langle\psi(T)|=| \psi(0)\rangle\langle\psi(0)|
$$

The period $T$ in here could be any time period. In the adiabatic approximation $T$ would be given by $T=\frac{2 \pi}{\omega}$.

It is not clear that solutions of (3.56) exist which fulfill the condition (3.57), however for a periodic Hamiltonian, like (3.1), we would expect that solutions with the same period exist. In addition we shall see that other cyclic evolutions exist and that even a time-independent Hamiltonian can have cyclic states.

We start with the adiabatic approximation and obtain the expression for the state vector by inserting the results, in particular (3.54) and (3.5) into (2.43)

$$
\psi(t) \stackrel{\text { adiabatic }}{\stackrel{\perp}{=}} e^{-i b t k} e^{i \gamma_{k}(t)}|k ; \theta, \omega t\rangle, \omega=\frac{2 \pi}{T}, \quad \gamma_{k}(t)=\gamma_{k}(\mathbf{C}) \frac{t}{T}
$$

This formula is an approximate equality indicated by the suffic "adiabatic" and, in general, not an equality (except for $\omega=0$ in which case it is the stationary state of a time independent Hamiltonian).

For the state vector after a period $T$ we obtain from (2.51):

$$
\psi(T) \stackrel{\text { adiabatic }}{\stackrel{1}{=}} e^{-i 2 \pi \frac{b}{\omega} k} e^{i \gamma_{k}(\mathbf{C})} \psi(0) ; \quad \psi(0)=|k ; \theta, 0\rangle .
$$

These expressions for the state vector are, as we showed in general in section 2 , incompatible with the Schrödinger equation (3.56) and can only be approximation.

But as all solutions of (3.56) are known ${ }^{1)}$ we can see whether there are cyclic solutions and select those. The exact solution of (3.56) with the initial state $\psi(0)$ is given by ${ }^{1) 2}$

$$
\psi(t)=U^{\dagger}(t) \psi(0)=e^{-i t \omega J_{3}} e^{-i t \Omega \mathbf{e} \cdot \mathbf{J}} \psi(0)
$$

The time evolution thus consists of a rotation of the sate $\psi(0)$ about e by $\Omega t$ followed by a rotation about $\mathbf{e}_{3}$ by $\omega t$ :

The unit vector $\mathbf{e}$ is shown in Fig. 3.1 and given by

$$
\mathbf{e}=\hat{\mathbf{R}}(\theta, 0)-\frac{\omega}{\Omega} \mathbf{e}_{3}=\frac{b}{\Omega}\left(\cos \theta-\frac{\omega}{b}\right) \mathbf{e}_{3}+\frac{b}{\Omega} \sin \theta \mathbf{e}_{1} \equiv \cos \tilde{\theta} \mathbf{e}_{3}+\sin \tilde{\theta} \mathbf{e}_{1}=\hat{\mathbf{R}}(\tilde{\theta}, 0)
$$

The new angle $\tilde{\theta}$ is shown in Fig. 3.1, and is defined by equation (3.61):

$$
\cos \tilde{\theta}=\frac{b}{\Omega}\left(\cos \theta-\frac{\omega}{b}\right) \quad \sin \tilde{\theta}=\frac{b}{\Omega} \sin \theta .
$$


The angular frequency $\Omega$ is obtained from the normalization condition $\mathbf{e}^{2}=1$ :

$$
\Omega=b\left(1+\frac{\omega}{b}\left(-2 \cos \theta+\frac{\omega}{b}\right)\right)^{\frac{1}{2}}
$$

These relations between $(\theta, \varphi)$ and $(\tilde{\theta}, \varphi)$ define a map $F: M=S^{2} \rightarrow S^{2}$, which in cartesian coordinates corresponding to the global orthonormal basis system $\left(\mathbf{e}_{1}, \mathbf{e}_{2}, \mathbf{e}_{3}\right)$ is given by

$$
F_{\nu}\left(x^{1}, x^{2}, x^{3}\right)=\frac{\left(x^{1}, x^{2},\left(x^{3}-\frac{\omega}{b}\right)\right)}{\sqrt{\left(x^{1}\right)^{2}+\left(x^{2}\right)^{2}+\left(x^{3}-\frac{\omega}{b}\right)^{2}}}
$$

It depends upon the physical parameter

$$
\nu \equiv \frac{\omega}{b}
$$

and is given in terms of the local spherical coordinates by

$$
F_{\nu}(R(\theta, \varphi))=F_{\nu}(\cos \theta, \varphi)=\left(\frac{\cos \theta-\nu}{\sqrt{\nu^{2}-2 \cos \theta \nu+1}}, \varphi\right)=(\cos \tilde{\theta}, \varphi) \equiv R(\tilde{\theta}, \varphi)
$$

The map $F_{\nu}$ is for $\frac{\omega}{b}<1$ a homeomorphism of $\mathcal{S}^{2}$ and consist of a translation in the $\mathbf{e}_{3}$ direction followed by a projection along $\mathbf{R}$ onto $\mathcal{S}^{2}$.

$F_{\nu}(\cos \theta, \varphi)$ is a continuous function over $(-1 \leq \cos \theta \leq 1,0 \leq \varphi<2 \pi)$ as long as $\nu \equiv \frac{\omega}{b}<1$. If $\nu \geq 1$ the map $F_{\nu}$ becomes discontinuous. We shall therefore restrict ourselves here to $\nu<1 ;{ }^{10}$ ) the adiabatic approximation is fulfilled for $\nu \ll 1$ which means that the external angular velocity $\omega$ for the precession of $\hat{\mathbf{R}}(\theta, \omega t)$ is small compared to the Larmor frequence $b$.

The Larmor frequency $b$ is the angular velocity with which the state $\psi(t)$ rotates about the direction of the magnetic field $\hat{\mathbf{R}}(\theta, 0)$ when $\omega \equiv \frac{2 \pi}{T}=0$ or $\nu=0$ :

$$
\psi(t)=e^{-i t b \hat{\mathbf{R}}(\theta, 0) \cdot \mathrm{J}} \psi(0) \quad \text { for } \quad \omega=0 \quad \text { or } \quad \nu=0
$$

This can be seen immediately if one rewrites (3.60) using (3.61) and (3.65):

$$
\psi(t)=e^{-i t b \nu J_{3}} \quad e^{-i t b\left(\mathbf{R}(\theta, 0) \cdot \mathrm{J}-\nu J_{3}\right)} \psi(0)
$$

Equation (3.67) gives the time evolution of a state vector $\psi(0)$ for the time independent Hamiltonian:

$$
h=b \hat{\mathbf{R}}(\theta, 0) \cdot \mathbf{J} \quad ; \quad \theta=\text { constant }
$$

In order to select from all the solutions (3.60) those, which describe cyclic evolution, (3.57), we have to choose in (3.60) for the initial state vector $\psi(0)$ an eigenvector of the operator

$$
U^{\dagger}(T)=e^{-i T \omega J_{3}} e^{-i T \Omega \hat{\mathbf{R}}(\bar{\theta}, 0) \cdot \mathbf{J}}
$$

Its eigenvalue $e^{-i \alpha_{w}}$ will then be the total phase factor in the cyclic evolution (3.57):

$$
U^{\dagger}(T) \psi(0)=e^{-i \alpha_{\psi}} \psi(0)=\psi(T)
$$


If $\psi(0)$ is eigenvector of $U^{\dagger}(T)$ ) with eigenvalue $e^{-i \alpha}$ it must also be eigenvector of $U(T)=e^{i T \Omega \vec{R} \cdot \vec{J}} e^{i T \omega J_{3}}$ with eigenvalue $e^{i \alpha}$. This requires that $e^{-i T \omega J_{3}}$ and $e^{-i T \Omega \mathbf{R}\left(\tilde{\theta}_{0}, 0\right) \cdot \mathbf{J}}$ commute:

$$
\left[e^{-i T \omega J_{3}}, e^{-i T \Omega \hat{\mathbf{R}}(\tilde{\theta}, 0) \cdot \mathrm{J}}\right]=0
$$

This can be fulfilled under the following two conditions:

$$
\begin{array}{lll}
\text { CLASS A : } & \omega T=2 \pi n ; \text { evolution period : } & T=\frac{2 \pi}{\omega} \\
\text { CLASS B : } & \Omega T=2 \pi n ; \text { evolution period : } & T=\frac{2 \pi}{\Omega}
\end{array}
$$

For both classes there will be a discrete set of cyclic solutions. A special case is $\omega=0$ in which case we have a time-independent Hamiltonian (3.68) with evolution (3.67). The class A cyclic solutions have the same period as the Hamiltonian and the adiabatic evolution. The Class B cyclic solutions have a period which is given by the intrinsic properties of the quantum system (the Larmor frequency $b$ modified by the precession frequency $\omega$ ).

We shall discuss here only the Class A cyclic evolutions because they are directly connected with the adiabatic cyclic changes.

The Class A cyclic solutions are obtained by finding the eigenvectors of the operator $U_{A}^{\dagger}(T)=e^{-i 2 \pi J_{3}} e^{-i T \Omega \hat{\mathbf{R}}(\bar{\theta}, 0) \cdot J}$. These eigenvectors of $U^{\dagger}(T)$ must be eigenvectors of both $e^{-i T \Omega \hat{\mathbf{R}}(\tilde{\theta}, 0) \mathrm{J}}$ and of $e^{-i 2 \pi J_{3}}$. From this it follows immediately that they must be eigenvectors of the operator:

$$
\frac{\Omega}{b} h(\tilde{\theta}, 0) \equiv \Omega \hat{\mathbf{R}}(\tilde{\theta}, 0) \cdot \mathbf{J} \quad \text { for } \quad \tilde{\theta} \neq \theta \quad \text { i.e. for } \omega \neq 0:
$$

These eigenvectors we call $\phi_{k}$ :

$$
\frac{\Omega}{b} h(\tilde{\theta}, 0) \phi_{k}=\Omega k \phi_{k} \quad ; \quad \mathbf{e} \cdot \mathbf{J} \phi_{k}=k \phi_{k}
$$

From (3.11) and (3.12) with $\theta$ replaced by $\tilde{\theta}$ and $\varphi$ by 0 it follows that $\phi_{\kappa}$ is (up to a phase factor if $\phi_{\kappa}$ is assumed to be normalized) given by

$$
\phi_{k}=|k ; \tilde{\theta}, 0\rangle=U(\tilde{\theta}, 0)\left|k ; \mathbf{e}_{3}\right\rangle=e^{-i \tilde{\theta} J_{2}}\left|k ; \mathbf{e}_{3}\right\rangle .
$$

To obtain the eigenvalue of $e^{-i 2 \pi J_{3}}$ we calculate using (3.15) for $\varphi=2 \pi$ :

$$
\begin{aligned}
e^{-i 2 \pi J_{3}} \phi_{k} & =e^{-i 2 \pi J_{3}}|k ; \tilde{\theta}, 0\rangle=e^{-i 2 \pi J_{3}} e^{-i \tilde{\theta} J_{2}}|k ; 0,0\rangle \\
& =e^{-i \tilde{\theta} J_{2}} e^{-i 2 \pi J_{3}}|k ; 0,0\rangle \\
& =e^{-i 2 \pi k}|k ; \tilde{\theta}, 0\rangle
\end{aligned}
$$

The initial state vectors which lead to a cyclic evolution with period $T=\frac{2 \pi}{\omega}$ are thus labelled by the integer or half-integer number $k$ and given by (3.76):

$$
\psi(0)=\phi_{k}=|k ; \tilde{\theta}, 0\rangle
$$


(after an arbitrary phase factor has been fixed). The total phase of these cyclic evolutions, i.e. the eigenvalues $e^{-i \alpha_{\psi}}$ of (3.70) are also labelled by $k$ and given by

$$
\psi(T)=U^{\dagger}(T) \psi(0)=e^{-i \alpha_{k}} \psi(0)=e^{-i 2 \pi k} e^{-i 2 \pi \frac{\Omega}{\omega} k} \psi(0)
$$

Thus the possible initial states of a cyclic evolution are very similar to the initial states of an adiabatic evolution which are given by the eigenvectors $|k ; \theta, 0\rangle$ of $h(0)=b \hat{\mathbf{R}}(\theta, 0) \cdot \mathbf{J}$. The initial states of the real cyclic evolution are instead given by the eigenvectors (3.76) of the operator

$$
\tilde{h}(\theta, 0) \equiv \frac{\Omega}{b} h(\tilde{\theta}, 0) \equiv \Omega \hat{\mathbf{R}}(\tilde{\theta}, 0) \cdot \mathbf{J}
$$

They are thus state with the component $k$ of angular momentum along the direction $\mathbf{e}=\hat{\mathbf{R}}(\tilde{\theta}, 0)$ rather than along the direction $\hat{\mathbf{R}}(\theta, 0)$ of the initial magnetic field, see Fig. 3.1. For large values of $\omega, \omega \approx b$ these two directions can be very different, but for $\nu=\frac{\omega}{b} \rightarrow 0$ they become the same.

We now have to determine the analogue of the single-valued eigenvectors $|k ; \mathbf{R}(t)\rangle=$ $|k ; \theta(t), \varphi(t)\rangle$ which are used in the calculation of the Berry connection and Berry phase. These vectors $\phi_{k}(t)$ lie on a curve above the closed curve

$$
\mathcal{C}: t \rightarrow|\psi(t)\rangle\langle\psi(t)|\quad ; \quad| \psi(T)\rangle\langle\psi(T)|=| \psi(0)\rangle\langle\psi(0)|
$$

They must fulfill:

$$
\phi_{k}(t)=\text { phase factor } \cdot \psi(t) ; \quad \phi_{k}(T)=\phi_{k}(0)
$$

There are many such vectors all differing by a phase transformation

$$
\phi_{k}(t) \rightarrow \phi_{k}^{\prime}(t)=e^{i \zeta_{k}(t)} \phi_{k}(t) \quad, \quad \zeta_{k}(0)=\zeta_{k}(T) \quad \bmod 2 \pi
$$

One, and the by far most obvious choice for $\phi_{k}(t)$ is

$$
\phi_{k}(t) \equiv U(\tilde{\theta}, \omega t)\left|k ; \mathbf{e}_{3}\right\rangle=|k ; \tilde{\theta}, \omega t\rangle=e^{-i \omega t J_{3}} e^{-i \tilde{\theta} J_{2}} e^{i \omega t k}\left|k, \mathbf{e}_{3}\right\rangle
$$

These single-valued basis vectors $|k ; \tilde{\theta}, \varphi=\omega t\rangle$ are eigenvectors of the operator

$$
\left.\tilde{h}(\theta, \varphi) \equiv \frac{\Omega}{b} h(\tilde{\theta}, \bar{\varphi})=\frac{\Omega}{b} h\left(F_{\nu}(\theta, \varphi)\right)=\Omega \hat{\mathbf{R}}(\tilde{\theta}, \varphi)\right) \cdot \mathbf{J}
$$

where $F_{\nu}$ is the function (3.66). The operator $\bar{h}(R)=\frac{\Omega}{b} h\left(F_{\nu}(R)\right)$ is not the Hamiltonian but another parameter dependent operator which can as well serve to define a basis system for the space $\mathcal{H}$. In terms of these known vectors $|k ; \tilde{\theta}, \varphi\rangle$ the cyclic solution of the Schrödinger equation (3.56) is given by

$$
\psi(t)=e^{-i \omega t k} e^{i \Omega t k}|k ; \tilde{\theta}, \omega t\rangle
$$

Therewith we have expressed the state vectors for cyclic time evolutions in terms of the known quantities $\omega, \Omega$ (of (3.63)) and $|k, \tilde{\theta}, \omega t\rangle$ (of (3.84). The equation (3.85) is the eneralization of the adiabatic "equality" (3.58) and (3.79) is the generalization of (3.59). However the phase factor in (3.85) and (3.79) is not in the form of a product 
of the dynamical and the geometrial part. In order to obtain that we have to calculate the dynamical or the geometrical phase factor separately.

\subsection{The Dynamical and the Geometrical Phase Factor for Non-Adiabatic} Evolution

The splitting of the phase factor into a "dynamical and a "geometrical" part can be approach from two different points of view. Either one gives an argument why a certain part is geometrical and obtains the dynamical phase as the difference between the total and geometrical phase, or one defines the dynamical phase and obtains the geometrical phase as the difference between the total and dynamical phase. We will define here the dynamical phase $\alpha_{k}^{d y n}$ and obtain the geometrical phase as a derived quantity.

$$
\alpha_{k}^{g e o m .}=\alpha_{k}-\alpha_{k}^{d y n}
$$

The dynamical phase for general cyclic evolution was defined by Aharonov and Anandan ${ }^{3}$ by

$$
\alpha_{k}^{d y n .} \equiv \int_{0}^{\hat{T}}\langle\psi(t)|h(t)| \psi(t)\rangle d t
$$

This is reasonable because one can show that the phase for the evolution of a stationary state (2.25) is given by (3.87) and because (3.87) goes into the dynamical phase for adiabatic cyclic evoluton given by (2.31).

It is straightforward to calculate the dynamical phase (3.87) for the Hamiltonian $(3.1)$ :

$$
\begin{aligned}
\alpha_{k}^{d y n} & \equiv \int_{0}^{T}\langle\psi(t)|h(t)| \psi(t)\rangle d t \\
& =k 2 \pi\left(\frac{\Omega}{\omega}+\cos \tilde{\theta}\right) .
\end{aligned}
$$

Using (3.86) we then calculate from (3.79)

$$
\alpha_{k}^{\text {geom. }}=k 2 \pi(1-\cos \tilde{\theta})=k 2 \pi\left(1+\frac{\omega}{\Omega}-\frac{b}{\Omega} \cos \theta\right) .
$$

This has the same form as (3.54) for the adiabatic case except that $\theta$ is replaced by $\tilde{\theta}$ of (3.62).

In the adiabatic approximation, $\nu=\frac{\omega}{b} \ll 1$, we can expand the expressions in (3.62) and (3.63) with respect to $\frac{\omega}{b}$ :

$$
\begin{aligned}
& \frac{\Omega}{b} \approx 1-\frac{\omega}{b} \cos \theta+\left(\frac{\omega}{b}\right)^{2} \\
& \sin \tilde{\theta} \approx \sin \theta+\frac{1}{2} \frac{\omega}{b} \sin 2 \theta ; \cos \tilde{\theta} \approx \cos \theta+\frac{1}{2} \frac{\omega}{b}(\cos 2 \theta-1)
\end{aligned}
$$

From this we obtain that the adiabatic approximation of the geometrical phase angle for general cyclic evolution (3.89) is identical with the Berry phase angle:

$$
-\alpha_{k}^{\text {geom }} \approx-2 \pi k(1-\cos \theta)=\gamma_{k}^{\text {Berry }}
$$


These results indicate that (3.86) with (3.87) is the appropriate choice of the geometrical phase also for the general cyclic evolution.

We now want to show that this phase angle can be obtained in the same way as Berry's phase for the adiabatic approximation from a gauge potential (connection).

In analogy to $(2.40),(2.42)$ we define the connection one-form by

$$
A^{\phi_{k}}=i\left\langle\phi_{k}(t)|d| \phi_{k}(t)\right\rangle=\langle k ; \tilde{\theta}, \omega t|d| k ; \tilde{\theta}, \omega t\rangle=\left\langle k ; \mathbf{e}_{3}\left|U^{\dagger}(\tilde{\theta}, \omega t) \frac{d}{d t} U(\tilde{\theta}, \omega t)\right| k ; \mathbf{e}_{3}\right\rangle d t .
$$

Here we have used in place of the single-valued eigenvectors of $h(t)$ in (2.42) the basis vectors (3.84) which are eigenvectors of another operator $\tilde{h}(R)$ of $(3.80 \mathrm{a})$. From the results (3.30) (with $\varphi=\omega t$ ) we obtain immediately:

$$
A^{\phi_{k}}=\left\langle k ; \mathbf{e}_{3}\left|-\left(J_{1} \cos \varphi+J_{2} \sin \varphi\right) \sin \tilde{\theta}+J_{3}(\cos \tilde{\theta}-1)\right| k ; \mathbf{e}_{3}\right\rangle d(\omega t)
$$

or

$$
A^{\phi_{k}}=-k(1-\cos \tilde{\theta}) d \omega t=-k\left(1-\frac{b}{\Omega} \cos \theta+\frac{\omega}{\Omega}\right) d \varphi(t)
$$

The curvature (field strength) that follows from this connection (gauge potential) is

$$
F=d A=-k \frac{\left(1-\frac{\omega}{b} \cos \theta\right)}{\left(\frac{\Omega}{b}\right)^{3}} \sin \theta d \theta \wedge d \varphi
$$

or

$$
F_{\theta \varphi}=-k \frac{1-\frac{\omega}{b} \cos \theta}{\left[1-\frac{\omega}{b} \cos +\left(\frac{\omega}{b}\right)^{2}\right]^{3 / 2}} \sin \theta ; \quad \mathbf{F}=-\frac{k}{r^{2}} \frac{\left(1-\frac{\omega}{b} \cos \theta\right)}{\left(\frac{\Omega}{b}\right)^{3}} \hat{\mathbf{R}}(\theta, \varphi)
$$

This is again the "monopole" type field as in (3.55) but with modified "monopole strength".

The connection one-form (3.95) has the same form as (3.34) for the adiabatic connection, except that here we have the angle $\tilde{\theta}$ which is not the angle $\theta$ for the direction of the magnetic field (parameter of the Hamiltonian).

We can now use the analog of the formula $\left(2.54^{\prime}\right)$ and (3.46) for the adiabatic approximation and define a "geometric phase" angle also for general cyclic evolution by

$$
\gamma_{k} \equiv \oint_{\mathbf{C}} A^{\phi_{k}}=\oint i\left\langle\phi_{k}(t)|d| \phi_{k}(t)\right\rangle
$$

Then we obtain with (3.95)

$$
\gamma_{k}=-k \int_{0}^{T}(1-\cos \tilde{\theta}) d \omega t=-k 2 \pi(1-\cos \tilde{\theta})=-2 \pi k\left(1-\frac{b}{\Omega} \cos \theta+\frac{\omega}{\Omega}\right) .
$$

This agrees with the result (3.89) obtained from (3.86), (3.87).

With these results we can rewrite the cyclic solution of the Schrödinger (3.85) in a form which completely resembles the adiabatic approximation (3.58):

$$
\psi(t)=e^{-i \omega t k(1-\cos \tilde{\theta})} e^{-i \omega t k\left(\frac{\Omega}{\omega}+\cos \tilde{\theta}\right)}|k ; \tilde{\theta}, \varphi\rangle=e^{i \gamma_{k}} e^{-i \alpha_{k}^{\mathrm{dyn}}}|k ; \tilde{\theta}, \varphi(t)\rangle .
$$


The concepts introduced in this section for the cyclic evolution (3.81) are the analogs of the concepts for the adiabatic approximation. The single-valued basis vectors $\phi_{k}$ are the analogs of the Hamiltonian eigenvectors $|k ; \theta, \varphi\rangle$. The connections $A^{\phi_{k}}$ of (3.94) are generalizations of the adiabatic connections (2.42). The geometric phase (3.97) is a generalization of the Berry phase (2.48).

The distinction between (3.99) and (3.58) is that in (3.99) we have an equality sign. This means that the the state vector $\psi(t)$ indeed "rotates" along the curve $t \rightarrow$ $\phi_{k}(t)$ described by the curve $t \rightarrow(\tilde{\theta}, \omega t)$ in parameter space, whereas the curve $t \rightarrow$ $(\theta, \omega t)$, describes the path of the external magnetic field, not of a state. For slow enough $\omega$ (however before $\omega$ becomes zero which is the stationary state development with $\left.\gamma_{k}=0\right)$ the curve (3.58) is "close enough" to (3.99), in order to provide an acceptable approximation for the geometric phase. But an exact adiabatic cyclic evolution does not exist.

As we mentioned above in addition to the cyclic evolutions of a pure state with period $T=\frac{2 \pi}{\omega}$ ( $\omega=$ precession frequency of the external magnetic field) there exist other cyclic evolutions with period $T=\frac{2 \pi}{\Omega}$ where $\Omega$ is given by (3.63) and therewith mainly determined by the Larmor frequency $b$ of the physical state (for $\omega=0$, which is the cyclic evolution in a constant magnetic field with time-independent Hamiltonian one has exactly $\Omega=b$ ). We shall not discuss these evolutions here.

The geometrical phase (3.98) is not purely geometrical in the way that (3.91) is, which only depends upon $\theta$ and is given solely by the path in the parameter space. The geometrical phase for general cyclic evolution also contains the parameters $\omega, b$ of the Hamiltonian. To fully justify the name geometric phase also for the phase (3.98) of non-adiabatic cyclic evolution we have to go from the geometry of the parameter space to the geometry of the space of physical states $\mathcal{P}(\mathcal{H})$. 


\section{References}

1. I.I. Rabi, N.F. Ramsey, J. Schwinger, Rev. Mod. Phys. 26, 167 (1954).

2. D.J. Fernandez, L.M. Nieto, L.M. del Olmo, M. Santander, Journal of Physics A (1992); S.J. Wang, Phys. Rev. A42, 5107 (1990).

3. See reference 9 , section 1 .

4. L.C. Biedenham and J.D. Louck, Angular Momentum in Quantum Physics, Addison-Wesley, 1981, Chapter 3.5 eq. (3.40)

5. The number $C_{1}=-\frac{1}{2 \pi} \int_{S^{2}} F$ is called the first Chern number of the monopole bundle $\left(U(1)=\mathcal{S}^{1}\right.$ bundle over the base space $\left.M=\mathcal{S}^{2}\right)$. The fiber bundles behind the geometric phase will be discussed in section 4 . The Chern number characterizes the bundle and takes the integer $2 k$ as shown above. If $k$ is the component of angular momentum in an irreducible representations of the $J_{i}$ then $k$ must be one of the integers or half integers $-j,-j+1, \ldots+j$. But there is also another proof which shows that $k$ must be integer or half integer, even if $\left|k ; e_{3}\right\rangle$ is not an eigenvector of $J^{2} . R_{i}$ are the eigenvalues of the operators $X_{i}$ which together with $J_{i}$ are the generators of the group $E_{3}$. Its unitary irreducible representations (see Appendix V.3 of reference 1 section 2) are characterized by the numbers $(k, \varepsilon)$ with $\varepsilon^{2}=R_{i} R_{i}(=1)$ and $k=$ eigenvalue $\frac{1}{\varepsilon} X_{i} J_{i}$, where $\varepsilon$ is any real positive number and $k$ is positive or negative integer or halfinteger including 0 . There are no unitary irreducible representations of $E_{3}$ for $2 k \neq$ integer.

6. P.A.M. Dirac, Proc. Roy. Soc. 133, 60(1931).

7. P. Goddard, D.I. Olive Rep. Prog. Phys. 41: 1357 (1978); L.C. Biedenham, J.D. Louck, (reference 4) Chapter 5.2; S. Coleman in The Unity of the Fundalmental Interactions ; H. Zichichi (editor) Plenum Press (1983) (Erice lecture (1981); A.B. Balachandran, G. Marmo, B.S. Skagerstam, A. Stern, Classical Topology and Quantum States Part I; World Scientific Publishers, (1991).

8. J.M. Leinaas, Physica Scripta 17, 483 (1978).

9. See reference 3 of section 1 and M.V. Berry and R. Lim, J. Phys. A23, L655 (1990); B. Zygelman, Phys. Rev. Lett. 64, 256 (1990), T. Pacher, C.A. Mead, L.S. Cederbaum, H. Köppel, J. Chem. Phys. 91, 7057 (1989); A. Bohm, B. Kendrick, Mark E. Loewe and L.J. Boya, J. Math. Phys. 33, 977 (1992); H.K. Lee and M. Rho, Hanyang University preprint HYUPT-92-07 (1992). 
4. The Anandan-Aharonov Phase for General Cyclic Evolution and its Relation to Berry's Phase - U(1) Bundles Over Parameter Space and Over the Space of Physical States

In this section we shall uncover the general pattern that underlies the results which we obtained for the specific example of the time-dependent Hamiltonian (3.1).

For a time-dependent cyclic Hamiltonian $h(t)=h(x(t)$ with a period $T$, whose time dependence may be given by a closed path $\mathrm{C}$ in the parameter space $M$ :

$$
\mathrm{C}: x(0) \rightarrow x(t) \rightarrow x(T)=x(0),
$$

There exists a number of cyclic paths

$$
\mathcal{C}: W(0) \rightarrow W(t)=|\psi(t)\rangle\langle\psi(t)| \rightarrow W(T)=W(0)
$$

in the space of pure physical states $\mathcal{P}(\mathcal{H})$ with the same period $T$. (There may be in addition other cylcic paths with different period.) In our example the number of cyclic path is countable and labelled by the half integer $k$,

$$
\mathcal{C}_{k}: W(t)=W_{k}(t)=\left|\phi_{k}(t)\right\rangle\left\langle\phi_{k}(t)\right|
$$

with $\phi_{k}(t)$ given by (3.84).

Associated with the curve $\mathcal{C}$ (or curves $\mathcal{C}_{k}$ ) in $\mathcal{P}(\mathcal{H})$ are three different kind of curves in the Hilbert space $\mathcal{H}$.

1. The curve

$$
C: \psi(0) \rightarrow \psi(t) \rightarrow \psi(T):=e^{-i \alpha \psi} \psi(0)
$$

is the curve of solutions of the Schrödinger equation with initial condition that leads to a cyclic solution.

2. The curve

$$
C^{\text {closed }}: \phi(x(0)) \rightarrow \phi(x(t)) \rightarrow \phi(x(T))=\phi(x(0))
$$

is a curve of smooth single-valued functions (continuousily differentiable) of vectors in $\mathcal{H}$ - that have the property $W(t)=|\phi(t)\rangle\langle\phi(t)|$. These single-valued vector functions are only determined up to a gauge transformation

$$
\phi(x(t)) \rightarrow \phi^{\prime}(x(t))=e^{i \zeta(x(t))} \phi(x(t)) ; e^{i \zeta(\tau)}=e^{i \zeta(0)} .
$$

In our example this closed curve of vectors in the Hilbert space $\mathcal{H}$ associated with the curve $\mathcal{C}_{k}$ in $\mathcal{P}(\mathcal{H})$ is

$$
C_{k}^{\text {closed }}:|k ; \tilde{\theta}, 0\rangle \rightarrow \phi_{k}(t)=|k ; \tilde{\theta}, \omega t\rangle \rightarrow|k ; \tilde{\theta}, \omega T\rangle=|k ; \tilde{\theta}, 0\rangle .
$$

where the vectors $\phi_{k}(t)$ are those of (3.84) and the gauge transformation (4.6) is the one in Eq. (3.83). The curve (4.4) in our example is given by the vectors (3.99).

In addition to the curves $C^{\text {closed }}$ and $C$ in $\mathcal{H}$ associated with a closed curve of pure physical states (solutions of the von Neumann-Schrödinger Eq. $(2.3 \mathrm{~b})$ ) $\mathcal{C}$ in $\mathcal{P}(\mathcal{H})$ we define $^{1)}$

3. The curve

$$
\tilde{C}: \tilde{\psi}(0) \rightarrow \tilde{\psi}(t)=e^{i \int_{0}^{t}\left\langle\psi(t)\left|h\left(t^{\prime}\right)\right| \psi\left(t^{\prime}\right)\right\rangle d t^{\prime}} \psi(t) \rightarrow \tilde{\psi}(T)
$$


where $\psi(t)$ is solution in (4.4).

ill three curves in $\mathcal{H}$ have the property

$$
|\psi(t)\rangle\langle\psi(t)|=| \phi(t)\rangle\langle\phi(t)|=| \tilde{\psi}(t)\rangle\langle\tilde{\psi}(t)|=W(t) \text { in } \mathcal{P}(\mathcal{H}) .
$$

For this reason we call these three curves in $\mathcal{H}$ lifts of the curve $\mathcal{C}$ in $\mathcal{P}(\mathcal{H})$, imagining that $t \rightarrow W(t)$ is a curve in some base space and $t \rightarrow \psi(t), t \rightarrow \phi(t), t \rightarrow \tilde{\psi}(t)$ are curves lying above these base space points. This is depicted in Fig. 4.1. The curve $C$ will be called the dynamical lift. The curve $C^{\text {closed }}$ defined by (4.8) will be called the closed lift. The curve $\widetilde{C}$ defined by (4.8) will be called the A-A lift. As we mentioned already, the closed lift is not uniquely determined but only up to a gauge transformation (4.6). The dynamical $l^{\wedge}$ of a given cur ve in the space of physical states $\mathcal{P}(\mathcal{H})$ is also not uniquely determined as we shall discuss instantly. However, the A-A lift of a given curve $\mathcal{C}$ is uniquely defined, it is identical with the horizontal lift of differential geometry, as we shall show below.

In our example (3.1) the A-A lift is given by

$$
\tilde{\psi}(t)=e^{i \int_{0}^{t}\left\langle\psi\left(t^{\prime}\right)\left|h\left(t^{\prime}\right)\right| \psi\left(t^{\prime}\right)\right\rangle t^{\prime}} \psi(t)=e^{i \alpha_{k}^{\mathrm{d} y n}} \psi(t)=e^{i \Omega k\left(1+\frac{\omega}{\Omega} \cos \bar{\theta}\right) t} \psi(t)
$$

It is not a closed curve but has the property

$$
\tilde{\mathcal{C}_{k}}: \tilde{\psi}(0) \rightarrow \tilde{\psi}(t) \rightarrow \tilde{\psi}(T)=e^{i \gamma_{k}} \tilde{\psi}(0),
$$

where $\gamma_{k}(T)=-\alpha_{k}^{\text {geom }}$ is the geometrical phase (3.89), (3.98).

The relation between the A-A lift, the dynamical lift and the closed lift in our example is given by:

$$
\tilde{\psi}_{k}(t)=e^{i \alpha_{k}^{d \nu n}} \psi(t)=e^{i \gamma_{k}(t)}|k ; \tilde{\theta}, \varphi(t)\rangle
$$

It is a straightforward calculation (Problem 4.1) ${ }^{*}$ to show that the A-A lift

$$
\tilde{\psi}(t)=e^{i \int_{0}^{t}\left\langle\psi\left(t^{\prime}\right)\left|h\left(t^{\prime}\right)\right| \psi\left(t^{\prime}\right)\right\rangle d t^{\prime}} \psi(t) .
$$

in general fulfills the following equation and initial condition if $\psi(t)$ fulfills the Schrödinger Eq. (2.3):

$$
\begin{aligned}
i \frac{d \tilde{\psi}(t)}{d t} & =(h(t)-\langle\psi(t)|h(t)| \psi(t)\rangle \mathbf{1}) \tilde{\psi}(t) \\
\tilde{\psi}(0) & =\psi(0)
\end{aligned}
$$

Taking the scalar pioduct of (4.14) with $\tilde{\psi}(t)$ or $\psi(t)$ we obtain

$$
\left\langle\tilde{\psi}(t)\left|\frac{d}{d t}\right| \tilde{\psi}(t)\right\rangle=\left\langle\psi(t)\left|\frac{d}{d t}\right| \tilde{\psi}(t)\right\rangle=0 .
$$

* Problem $;.$. Show that the vector $\tilde{\psi}(t)$ defined by (4.13) fulfills the equations (4.14) if $\psi(t)$ fulfills the Schrödinger equation $i \frac{d \psi(t)}{d t}=h(t) \psi(t)$. 
This means that the tangent vector $\frac{d}{d t} \tilde{\psi}(t)$ of $\tilde{\psi}(t)$ is orthogonal (in the Hilbert space sense) to $\psi(t)$ and to $\tilde{\psi}(t)$.

The dynamical lift $C$ is uniquely determined by the Hamiltonian $h(t)$, but not uniquely determined by the physical problem. A simple substitution

$$
h(t) \rightarrow h^{\prime}(t)=h(t)-\kappa(t) \mathbf{1} \quad \text { with } \kappa(t) \in \mathbb{R}
$$

leais to a new Hamiltonian $h^{\prime}(t)$ which describes the same physics. It has the same (closed) curves of physical states $t \rightarrow W(t)=|\psi(t)\rangle\langle\psi(t)|$ in the projective Hilbert space $\mathcal{P}(\mathcal{H})$ as $h(t)$ (Problem 4.2).* But $h(t)$ and $h^{\prime}(t)$ have different dynamical lifts $t \rightarrow \psi(t)$ and $t \rightarrow \psi^{\prime}(t)$ (Problem 4.3). Also, two Hamiltonians $h(t)$ and $h^{\prime}(t)$ which have the same curves of physical states $t \rightarrow|\psi(t)\rangle\langle\psi(t)|$ and $t \rightarrow\left|\psi^{\prime}(t)\right\rangle\left\langle\psi^{\prime}(t)\right|$ differ by a multiple of the unit operator $\kappa(t) 1$. We are looking for a lift which is unique for a given physical problem, i.e. a lift that is uniquely associated to a closed curve $t \rightarrow|\psi(t)\rangle\langle\psi(t)|$ in $\mathcal{P}(\mathcal{H})$. Such a lift happens to be the A-A lift $\tilde{C}: t \rightarrow \tilde{\psi}(t)$. This can be seen in the following way: In addition to $\tilde{\psi}(t)$ defined by (4.13) using $h(t)$ and $\psi(t)$, (where $\psi(t)$ is the solution of the Schrödinger equation (2.3a) with Hamiltonian $h(t)$ ), one defines $\tilde{\psi}^{\prime}(t)$ by

$$
\tilde{\psi}^{\prime}(t)=e^{i \int_{0}^{t}\left\langle\psi^{\prime}\left(t^{\prime}\right)\left|h^{\prime}\left(t^{\prime}\right)\right| \psi^{\prime}\left(t^{\prime}\right)\right\rangle d t^{\prime}} \psi^{\prime}(t)
$$

where $\psi^{\prime}(t)$ is a solution of the Schrödinger equation with $h^{\prime}(t)$ :

$$
i \frac{d \psi^{\prime}(t)}{d t}=h^{\prime}(t) \psi^{\prime}(t)
$$

Then one can show that $\tilde{\psi}^{\prime}(t)=\tilde{\psi}(t)$ (Problem 4.4).*

We will now discuss the generalizations of (4.7), the closed lifts of the curve $\mathcal{C}$. As seen from (3.97) the $\phi_{k}(t)$ are the quantities in terms of which the geometric phase can be calculated if one does not have a solution $\psi(t)$ of the Schrödinger equation. There are various ways to obtain these single valued vectors $\phi(t) \epsilon \mathcal{H} .^{2}$ In general they are curves of local "sections". Sections are defined as continuously differentiable maps of an open

* Problem 4.2. Show that the closed path $\mathcal{C}: t \rightarrow|\psi(t)\rangle\langle\psi(t)|$ in the space of physical states $\mathcal{P}(\mathcal{H})$ which is generated by a Hamiltonian $h(t)$ is not affected by a change of the Hamiltonian $h(t) \rightarrow h^{\prime}(t)=h(t)-\kappa(t) 1$ where $\kappa(t) \in \mathbb{R}$.

Problem 4.9. Show that the dynamical lift of the closed path $\mathcal{C}$ in $\mathcal{P}(\mathcal{H})$ chianges under the substitution $h(t) \rightarrow h^{\prime}(t)=\kappa(t) 1$ and calculate the phase difference between the dynamical lift $\psi(t)$ belonging to $h(t)$ and the dynamical lift $\psi^{\prime}(t)$ belonging to $h^{\prime}(t)$.

Problem 4.4. Show that the horizontal lift $\tilde{C}$ of a closed path $\mathcal{C}$ in $\mathcal{P}(\mathcal{H})$, defined in terms of the dynamical lift $\psi(t)$ by

$$
\tilde{\psi}(t)=\exp \left(i \int_{0}^{t}\left\langle\psi\left(t^{\prime}\right)\left|h\left(t^{\prime}\right)\right| \psi\left(t^{\prime}\right)\right\rangle d t^{\prime}\right) \psi(t)
$$

is not affected by the substitution

$$
h(t) \rightarrow h^{\prime}(t)=h(t)-\kappa(t) 1 .
$$


area $O \subset \mathcal{P H}$ into $\mathcal{H}: \phi: O \rightarrow \mathcal{H}$. We will assume that our curve $\mathcal{C}$ lies in such an open area $O$. Then for any closed curve $\mathcal{C}: t \rightarrow W(t)$ one has the single valued lift

$$
t \rightarrow \phi(t)=\phi(W(t)), \quad W(t)=|\phi(t)\rangle\langle\phi(t)| ; \quad \phi(T)=\phi(0)
$$

(determined only up to a gauge transformation (4.6)). The problem is, of course, to determine a "section" or one of these single-valued lifts for a given time dependent Hamiltonian. But if one knows $\phi$ one can use it to calculate the geometric phase.

Since $\psi(t), \tilde{\psi}(t)$ and any $\phi(t)$ are lifts of the same closed curve $W(t)$, there must exist a phase factor $\omega(t)$ such that

$$
\tilde{\psi}(t)=\omega(t) \phi(t)
$$

We now calculate $\omega(t)$. Taking the derivative of (4.18), we get,

$$
\frac{d \tilde{\psi}(t)}{d t}=\frac{d \omega(t)}{d t} \phi(t)+\omega(t) \frac{d \phi(t)}{d t}
$$

Taking the scalar product of this with $\hat{\psi}(t)$, we obtain

$$
\omega(t) \frac{d \omega(t)}{d t}+\omega^{2}(t)\left(\phi(t), \frac{d \phi(t)}{d t}\right)=\left(\tilde{\psi}(t), \frac{d \tilde{\psi}(t)}{d t}\right)=0
$$

where the last equality holds because of (4.15). Thus

$$
\frac{1}{\omega(t)} \frac{d \omega(t)}{d t}=-\left(\phi(t), \frac{d \phi(t)}{d t}\right) .
$$

Integrating this we obtain

$$
\frac{\omega(t)}{\omega(0)}=e^{-\int_{0}^{t}\left(\phi\left(t^{\prime}\right), \frac{d \phi\left(t^{\prime}\right)}{d t^{\prime}}\right) d t^{\prime}} .
$$

This phase factor we call $e^{i \gamma(t)}$ and write (4.18) as

$$
\tilde{\psi}(t)=\omega(0) e^{i \int_{0}^{t} i\left(\phi\left(t^{\prime}\right), \frac{d}{d t^{\prime}} \phi\left(t^{\prime}\right)\right) d t^{\prime}} \phi(t) \equiv \omega(0) e^{i \gamma(t)} \phi(t) .
$$

If we choose the arbitrary constant phase $\omega(0)$ again such that $\tilde{\psi}(0)=\psi(0)=\phi(0)$, i.e. choose $\omega(0)=1$, then we have

$$
\tilde{\psi}(t)=e^{i \gamma(t)} \phi(t)
$$

which is the generalization of (4.12). With the general definition (4.13) of the A-A lift we then have

$$
\psi(t)=e^{-i \int_{0}^{t}\left(\psi\left(t^{\prime}\right), h\left(t^{\prime}\right) \psi\left(t^{\prime}\right)\right) d t^{\prime}} e^{i \gamma(t)} \phi(t) .
$$

This is the general relation between the solution of the Schrödinger equation $\psi(t)$ and the closed lift $\phi(t)$. The equation (2.43) is the adiabatic approximation of this, and (4.12) is the special case of this for the spinning quantum system in the precessing external magnetic field. The adiabatic approximation uses in place of the single-valued lifts $\phi^{\prime}(t)$ the single-valued eigenvectors of $h(R(t)$ which are more easily accessible than 
the $\phi(t)$ (by solving (2.4)). For the closed path $\mathcal{C}$ in $\mathcal{P}(\mathcal{H})$ the phase angle is (according (4.20))

$$
\gamma(T)=\gamma(\mathcal{C})=\oint_{0}^{T} i\left\langle\phi(t)\left|\frac{d}{d t}\right| \phi(t)\right\rangle d t=\oint i\langle\phi|d| \phi\rangle \bmod 2 \pi
$$

In here $\phi$ is any of the closed lifts of $\mathcal{C}$.

The phase angle $\gamma(T)$ is independent of the choice of the parameterization (the speed with which $\phi(t)$ traverses its closed path). It is gauge invariant. It is independent of the choice of the Hamiltonian as long as these Hamiltonians described the same closed path $\mathcal{C}$ in $\mathcal{P}(\mathcal{H})$. It depends upon the closed curve $\mathcal{C}$. It also depends upon the class of Hamiltonians that are connected to each other by the transformation (4.16), but only to the extent that the possible curves $\mathcal{C}$ are determined by $h(t)$. It can thus be considered a "geometric" property of the curve $\mathcal{C}$ in the space of physical states $\mathcal{P}(\mathcal{H})$. Thus the name geometric phase.

The one-form, defined in analogy to the adiabatic connection (2.42) and in analogy to $(3.92)$

$$
A^{\phi}=i\langle\phi|d| \phi\rangle
$$

transforms under a gauge transformation (4.6) as (Problem 4.5):*

$$
A^{\phi} \rightarrow A^{\phi^{\prime}}=A^{\phi}-d \zeta .
$$

This one-form is called the (non-adiabatic) connection form. The formula (4.24) for the connection was obtained from the requirement that $\tilde{\psi}(t)$ was the A-A lift i.e. the lift fulfilling (4.15) (which in turn followed from its definition (4.13)). This was the only possible definition of a lift which depends only upon the physics of the problem (and not upon the arbitrary choice of the Hamiltonian within the class connected by the transformation (4.16)) and which is uniquely determined by the closed curve $\mathcal{C}$ in the space of physical states. The A-A lift $\tilde{\psi}$ is therefore uniquely determined by the requirement that it be a property of the physical quantities $W(t)$ only.

From (4.21) and (4.17) it follows immediately that

$$
\tilde{\psi}(T)=e^{i \gamma(\mathcal{C})} \tilde{\psi}(0)=e^{i \gamma(\mathcal{C})} \psi(0) .
$$

Using (4.13) one obtains then for the cyclic evolution of a state vector:

$$
\psi(T)=e^{-i / \hbar \int_{0}^{T}\langle\psi(t)|h(t)| \psi(t)\rangle d t^{\prime}} e^{i \gamma(\mathcal{C})} \psi(0) .
$$

This is the general relation of which (2.51) is the adiabatic approximation.

As the physics is not in $\psi$ (which depends upon some arbitrary choice of the Hamiltonian) but in $\bar{\psi}$ (which is uniquely determined by the physical state $|\psi(t)\rangle\langle\psi(t)|$ ), the so-called geometrical phase $\gamma(\mathcal{C})$ is really the phase that is of physical importance.

A graphical representation of the above described situation for a general cyclic evolution is shown in Fig. 4.1. It shows a "base space" with a closed curve in it. The closed curve $\mathcal{C}$ represents the cyclic evolution of a pure state $W(t)$, and the base space

* Problem 4.5. Find the transformation of the connection one-form $A^{\phi} \equiv i\langle\phi|d| \phi\rangle$ when the section $\phi$ undergoes the phase transformation $\phi(t) \rightarrow \phi^{\prime}(t)=e^{i \zeta(t)} \phi(t)$. 
represents the space of physical states $\mathcal{P}(\mathcal{H})$. Above the closed curve are shown the three lifts $C^{\text {closed }}, \tilde{C}$ and $C$ in the Hilbert space $\mathcal{H}$ (depicted in the figure by the threedimensional space). Also shown is one "fibre" above the base point $W(0)$ (depicted by the positive $z$-axis with the $z$ coordinate representing the phase angle modulo $2 \pi$ or the element $e^{i \zeta(0)}$ of the gauge group $U(1)$ ). The "fibre" in this case (though it has been drawn as a straight line) is given by the circle of unit radius $S^{1}$ or by the group $U(1)$. We can attach a copy of this $S^{1}$ not only to the point $W(0)$ but to every one-dimensional projection operator $\Lambda \in \mathcal{P}(\mathcal{H})$. In this way we get a bundle of $U(1)$ fibres attached (which means loosely associated) to each point of $\mathcal{P}(\mathcal{H})$.

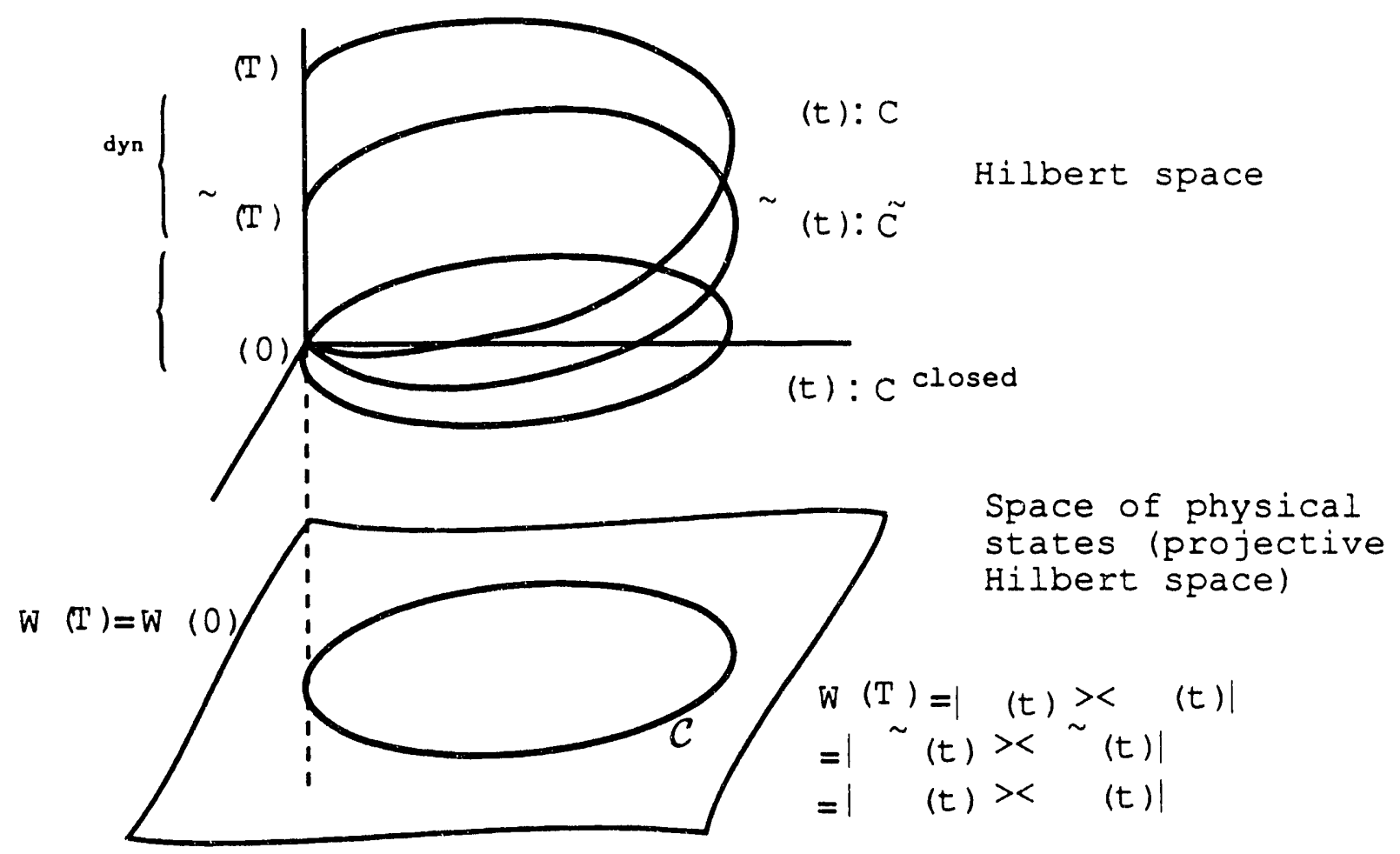

Figure 4.1. Closed path in the space of physical states and its lifts. 
The mathematical structure that we have encountered here is an example of a principal fibre bundle. The formal definition of a fibre bundle and a principal fibre bundle will be given in the Appendix. Here we will adapt the mathematical notions defined and developed in the Appendix to the $U(1)$ bundles underlying the geometric phase. $^{3}$

In general a fibre bundle consists of a total space, $E$, (topological space or differentiable manifold), a base space $M$, a fiber space $F$, a structure group $G$ acting on the fiber and a projection map $\pi$

$$
E: F \rightarrow E \stackrel{\pi}{\longrightarrow} M
$$

Often, but not always, one denotes the fibre bundle and the total space by the same letter $E$. If the structure group $G$ is a Lie group of diffeomorphisms of $F$ with $G$ being the same manifold as $F$ itself ( $G$ acting on itself by left or right translations) then the bundle is called a principal fiber bundle.

The set of all one-dimensional projection operators of $\mathcal{H}$ forms the projective space $\mathcal{P}(\mathcal{H})=\mathbb{C} P^{\infty}$ (If $\mathcal{H}$ is finite dimensional, i.e. the $N$ dimensional space $\mathbb{C}^{N}$, then $\mathcal{P}(\mathcal{H})$ is the projective space $\left.\mathbb{C} P^{N-1}\right)$. The set of all state vectors is the Hilbert space ${ }^{4)}$ without the zero vectors $\mathcal{H}-\{0\}$. If we restrict ourselves to normalized state vectors, which we can do for unitary time development, then we have to consider only the unit sphere $\mathcal{S}(\mathcal{H})=\mathcal{S}^{\infty}=\{\psi \in \mathcal{H} ;\|\psi\|=1\}$ in $\mathcal{H}$ (If $\mathcal{H}$ is $N$ dimensional then the unit vectors form $\left.\mathcal{S}^{2 N-1}\right)$. To each state $|\psi\rangle\langle\psi| \in \mathcal{P}(\mathcal{H})$ there corresponds a set of vectors $\left\{e^{i \zeta} \psi ; \psi \in \mathcal{S}^{\infty} ; e^{i \zeta} \in U(1)\right\}$ which have all the same projection operator: $\left|e^{i \zeta} \psi\right\rangle\left\langle e^{i \zeta} \psi|=| \psi\right\rangle\langle\psi|$. Therefore we have a map, the projection map

$$
\pi: \mathcal{S}(\mathcal{H}) \rightarrow \mathcal{P}(\mathcal{H}) \quad\left(\text { or } \quad \pi: \mathcal{S}^{2 N-1} \rightarrow \mathbb{C} P^{N-1}\right),
$$

with the property

$$
\pi\left(e^{i \zeta} \psi\right)=\pi(|\psi\rangle)=|\psi\rangle\langle\psi|
$$

The inverse image $\pi^{-1}(|\psi\rangle\langle\psi|)$ is called the fiber over $|\psi\rangle\langle\psi|$. For any $|\chi\rangle\langle\chi| \in \mathcal{P}(\mathcal{H})$ the fibres $\pi^{-1}(|\chi\rangle\langle\chi|), \chi \in(\mathcal{H})$ are essentially the same. They are given by $\left\{|\chi\rangle e^{i \zeta}, 0 \leq\right.$ $\zeta<2 \pi\}=F_{|\chi\rangle}$, the fiber over $|\chi\rangle$. The fibres are all homeomorphic (related by a continuous map whose inverse is also continuous) or diffeomorphic to $U(1)=\left\{e^{i \zeta}\right\}$, called the typical fibre. Thus we have

$\begin{array}{lll}\mathcal{P}(\mathcal{H})=\mathbb{C} P^{\infty} & \left(\text { or } \mathbb{C} P^{N-1}\right) & \text { as the base space } M, \\ \mathcal{S}(\mathcal{H})=\mathcal{S}^{\infty} & \left(\text { or } \mathcal{S}^{2 N-1}\right) & \text { as the total space } E, \\ \pi \text { of }(4.28) & & \text { as the projection map } \pi, \text { and } \\ U(1) & & \text { as the fibre } F \\ U(1) & \text { also as the structure group } G .\end{array}$

The aggregate

$$
\eta: U(1) \rightarrow \mathcal{S}(\mathcal{H}) \stackrel{\pi}{\rightarrow} \mathcal{P}(\mathcal{H}) \quad \text { (or } U(1) \rightarrow \mathcal{S}^{2 N-1} \rightarrow \mathbb{C} P^{N-1} \text { ) }
$$

is our principal fibre bundle (principal bundle because $F=G=U(1)$.

The projection map $\pi$ leads from a curve $t \rightarrow p(t) \in E$ in the bundle to a curve $t \rightarrow m(t)=\pi(p(t))$ in the base space. The curve $p(t)$ is called a lift of the curve $m(t)$ in the base space $M$. Thus the curves (4.4), (4.5) and (4.8) are examples of different lifts 
of the curve (4.2) in $\mathbb{C} P^{\infty}$. We mentioned that of these three kind of lifts only the lift (4.8), which we called the A-A lift, was uniquely defined. We shall now show that this lift is related to the geometrical notion of parallelism which at first sight has nothing to do with the definition (4.13) (by Anandan and Aharonov) ${ }^{1}$. Indeed it will turn out that $\bar{\psi}(t) \in \mathcal{H}$ is the point obtained from $\bar{\psi}(0)=\psi(0)$ by "parallel transport" along the curve $\mathcal{C}$ in $\mathcal{P}(\mathcal{H})$. This is also expressed by saying that the curve $\tilde{C}$ is the "horizontal lift" of the curve $\mathcal{C}$.

In order to discuss parallelism between a smooth curve in the base space $\mathcal{C}: t \rightarrow$ $|\chi(t)\rangle\langle\chi(t)|$ and one of its lifts $C: t \rightarrow \chi(t)\rangle$ we have to define an additional geometric structure for the fibre bundle, called a connection on $\eta$. For a given bundle one can define different connections and get different meanings of parallel transport. For the $U(1)$-bundle over the projective space $\mathcal{P}(\mathcal{H}), \eta$, the mathematically natural connection is the Stiefel connection which has been defined in mathematics long before ${ }^{5)}$ the lift (4.8) was introduced in quantum physics. ${ }^{1}$ That the lift which is horizontal with respect to the Stiefel connection is identical to the A-A lift (4.13), (which provides the quantal phase factor, (4.25), that can be measured in an interference experiment) is one of the more remarkable example of the "unreasonable effectiveness of mathematics in natural science" (Wigner). This was probably the main reason for the popularity which Berry's phase acquired among mathematical physicists.

In order to define the connection and therewith the horizontal lift for the fibre bundle $E$, we consider the tangent space $T_{p} E$ at the point $p \in E$. This tangent space can be decomposed into two subspaces: The vertrical subspace is defined as the subspace of those vectors which are tangent to the fibre through $p$ (tangent vectors to all possible curves along the fibre only). This subspace is denoted by $V_{p} E$; it is determined by the fiber. For the principal bundle it is isomorphic to the Lie algebra of the structure group $G$ (in our case of $G=U(1)$ it is generated by $i$ ) and has the dimension of the fibre (= structure group $G$ ).

The horizontal subspace, denoted by $H_{p} E$, is the space chosen to be the supplementary linear subspace in $T_{p} E$ to $V_{p} E$ :

$$
T_{p} E=V_{p} E+H_{p} E
$$

where + is the direct sum of linear spaces. In other words, every $\tau \in T_{p} E$ is given by

$$
\tau=\operatorname{ver}(\tau)+\operatorname{hor}(\tau) \quad ; \quad \operatorname{ver} \tau \in V_{p} E, \operatorname{hor} \tau \in H_{p} E
$$

The horizontal subspace is isomorphic to the tangent space $T_{\pi(p)} M$ on the base space $M$ at ihe point to which $p$ projects, $\pi(p)$.

The assignment to each point $p \in E$ of a horizontal subspace $H_{p} E$ of $T_{p} E$ such that (4.30) and (4.31) is fulfilled, is called a connection.

After the choice of a connection the horizontal lift can be defined: Let $t \rightarrow m(t)$ be a curve in $M$ then its horizontal lift $p(t)$ is the curve in $E$ for which $\pi(p(t))=m(t)$ and for which all vectors tangent to $p(t)$ are in the horizontal space $H_{p(t)} E$.

We will now choose the connection for our particular bundle $\eta$ of (4.29). This means we have to assign the horizontal subspace $H_{|\psi\rangle} S(\mathcal{H})$ to every $|\psi\rangle \in \mathcal{S}(\mathcal{H})$. We use the scalar product of $\mathcal{H}$ to make this assignment. 
In our case $\psi(t)$ is in the fiber over $|\psi(t)\rangle\langle\psi(t)|$ for every fixed $t$. The vector $\frac{d}{d t} \psi(t)$ is the tangent vector: $\frac{d}{d t} \psi(t) \in T_{\psi(t)} \mathcal{S}(\mathcal{H})$. We now define the horizontal lift of $(4.2)$ :

$$
\hat{\psi}(t) \in \pi^{-1}(|\psi(t)\rangle\langle\psi(t)|)=\pi^{-1}(W(t)) \text { is a horizontal lift }
$$

i.e.

iff

$$
\frac{d}{d t} \hat{\psi}(t) \in H_{\hat{\psi}(t)} S(\mathcal{H})
$$

$$
\left\langle\hat{\psi}(t)\left|\frac{d}{d t}\right| \hat{\psi}(t)\right\rangle=\left\langle\psi(t)\left|\frac{d}{d t}\right| \hat{\psi}(t)\right\rangle=0
$$

In other words: the curve $t \rightarrow \hat{\psi}(t)$ is a horizontal lift of the curve $t \rightarrow W(t)$ if the vector $\frac{d}{d t} \hat{\psi}(t) \in \mathcal{H}$ is orthogonal in the Hilbert space sense to the elements of the fiber.

Note that $\hat{\psi}(t)$ are elements of $\mathcal{S}(\mathcal{H}) \subset \mathcal{H}$, and $\frac{d \hat{\psi}(t)}{d t}$ are elements of $T \mathcal{S}(\mathcal{H})$. For a general fiber bundle the elements of $T E$ are not elements of $E$. But in the particular case of (4.29) the $\frac{d}{d t} \hat{\psi}(t)$ (as the derivative of a parameter dependent Hilbert space vector) is again an element of $\mathcal{H}: T \mathcal{S}(\mathcal{H}) \subset \mathcal{H}$. Thus (4.32), relating elements of $T \mathcal{S}(\mathcal{H})$ with elements of $\mathcal{S}(\mathcal{H})$ makes sense. The assignment of a horizontal subspace by (4.32) is called the Stiefel connection. ${ }^{5}$ )

For a given curve $m(t)$ in the base space and any initial point $p_{0}=p(0)$ in $E$ (with $\pi\left(p_{0}\right)=m(0)$ ) there exists exactly one horizontal lift $\mathrm{p}(\mathrm{t})$. For a closed curve $t \rightarrow m(t) ; m(0)=m(T)=m_{0}$, the horizontal lift starting from $p(0)$ will end at a point $p(T)=\pi^{-1}\left(m_{0}\right)$ in the fiber above $m_{0}$. As every point of the fiber can be reached from $p(0)$ by a transformation with exactly one element $g$ of the structure group $G$ we must have that $p(T)=p(0) g$. The set of all the $g$ for a given starting point $p(0)$ forms a subgroup of $G$ which is called the holonomy group of $p(0)$. In some cases, which we shall restrict ourselves to, the $r$ lonomy group of some point $p_{0} \in E$ will be identical to the structure group $G$. Then (if $M$ is connected) the holonomy group is the same for all $p \in E$. In this case any two points of $E$ can be joined by a horizontal curve in $E$. A connection, for which the holonomy groups of any $p \in E$ is the structure group $G$, is called irreducible connection.

After this review of general differential geometrical results we will turn to our specific fiber bundle $\eta$ and the closed curve (4.2) in the space of physical states $\mathcal{P}(\mathcal{H})$. We want to find the horizontal lift $t \rightarrow \hat{\psi}(t)$ with respect to the Stiefel connection. According to the general results above $\hat{\psi}(t)$ is uniquely determined for a given starting point $\hat{\psi}(0)$ for which we will usually chouse the initial state vector $\hat{\psi}(0)=\psi(0)$. The phase factor $e^{i \hat{\gamma}}$ :

$$
\hat{\psi}(T)=e^{i \hat{\gamma}} \hat{\psi}(0)
$$

is then an element of the holonomy group (which here is identical with the structure group $U(1)$ ). The phase factor $e^{i \hat{\gamma}}$ is uniquely determined for a given $\hat{\psi}(0)$. It is called the holonomy (anholonomy*) of the closed path $\mathcal{C}: \hat{\gamma}=\hat{\gamma}(\psi(0))=\hat{\gamma}(\mathcal{C})$.

Another description of the connection than the assignment (4.32a) of a horizontal subspace $H_{\hat{\psi}(t)} S(\mathcal{H})$ is the description of the connection in terms of a connection-oneform or the connection coefficients (gauge potential, see appendix). A connection form 
is a one-form $\mathcal{A}$ with values in the Lie algebra of the structure group $\mathrm{G}$ (which is isomorphic to the vertical subspace $V_{p} E$ ) such that the holonomy can be written as

$$
\hat{\gamma}=\oint_{\mathcal{C}} \mathcal{A} \quad(\hat{\gamma}=\mathcal{T} \oint \mathcal{A} \text { if } \mathrm{G} \text { is non - Abelian }) .
$$

where $\mathcal{C}$ is the closed path (4.2) in the base space. In order to obtain the connection one-form $\mathcal{A}$ that leads by (4.34) to the holonomy in (4.33) we start with a section. A section of a bundle (Appendix) is a continuous $\left(C^{\infty}\right)$ map $\phi: M \rightarrow E$ which assigns a preferred point $\phi(m)$ on the fibre $\pi^{-1}(m)$ to every $m \in M$. Thus the section undoes in a continuous way what the projection has done: $\pi \phi(m)=m$ for all $m \in M$. Global sections (for all $m \in M$ ) of principal bundles only exist if the bundle is trivial (i.e. homeomorphic to $M \times G$ ). For the nontrivial bundle $\eta$ there exist only local sections. A local section over a patch $O \subset M$ is a map $\phi: O \rightarrow E$ such that $\pi \phi(m)=m$ for $m \in O$. For $\eta$ (like for every principal bundle) there is always a covering $\left\{O_{\alpha}\right\}$ of $M$ such that every $O_{\alpha}$ admits a local section $\phi^{(\alpha)}$. Given a local section $\phi^{(\alpha)}$, one can detine another section $\phi^{(\alpha)^{\prime}}$ by

$$
\phi^{(\alpha)^{\prime}}(m)=\phi^{(\alpha)}(m) g(m)
$$

where $g(m) \in G=$ structure group. This is the general gauge transformation which has occurred in various specific forms before, $\mathrm{cf}(4.6),(3.83),(3.19),(2.12)$. A local section $\phi$ of $O$ maps a closed path $t \rightarrow W(t)$ in $\mathbb{C} P^{\infty}(W(0)=W(T))$ into a closed path in $\mathcal{S}(\mathcal{H})$ :

$$
t \rightarrow \phi(t) ; \phi(0)=\phi(T) .
$$

A local section thus provides a closed lift (4.5) of the curve $\mathcal{C}$ in $\mathcal{P}(\mathcal{H})$.

We now obtain (a local representation of) the connection one-form $\mathcal{A}$ in terms of the local section.

We choose a local section $\phi=\phi(W)$ for the patch that contains the closed path $\mathcal{C}$ of (4.2). The problem in each particular case is, of course, to find such a local section for a given time-dependent Hamiltonian $h(t)$. (In our example of section 3 we obtained the section $|k ; \tilde{\theta}, \varphi\rangle$ of (3.84) (local in the northern hemisphere) essentially by solving the problem explicitly.) With this section we lift the closed path $\mathcal{C}$ into a closed path of this section

$$
C^{\text {closed }}: \phi(t)=\phi(W(t)) \quad ; \quad \phi(T)=\phi(0)
$$

As $\hat{\psi}(t)$ is the horizontal lift of the same path (4.2) we can express the open path $t \rightarrow \hat{\psi}(t)$ in $S(\mathcal{H})$ in terms of the closed path $t \rightarrow \phi(t)$ in $S(\mathcal{H})$ by

$$
\hat{\psi}(t)=e^{i f(t)} \phi(t)
$$

where $f(t)$ is a smooth (single-valued) functon. As $\phi(T)=\phi(0)$ we obtain

$$
\hat{\psi}(T)=e^{i(f(T)-f(0))} \hat{\psi}(0)
$$

Comparing this with the definition of the holonomy (4.33) we see that

$$
\hat{\gamma}=f(T)-f(0)
$$


This we can calculate now by substituting (4.38) into the defining relation (4.32b) of the horizontal lift and integrating. This yields:

$$
\hat{\gamma}=\int_{0}^{T} i\left\langle\phi(t)\left|\frac{d}{d t}\right| \phi(t)\right\rangle d t=\int_{0}^{T} i\langle\phi|d| \phi\rangle
$$

Thus the connection one-form given by the assignment of the horizontal subspace (4.32) is:

$$
\mathcal{A}=i\langle\phi|d| \phi\rangle .
$$

This connection one-form is also called the Stiefel connection (of the $U(1)$ bundle $\eta$ over $\mathbb{C} P^{N-1}$ or $\mathbb{C} P^{\infty}$ ). The bundle space $\mathcal{S}\left(\mathcal{C}^{N}\right)=\mathcal{S}^{2 N-1}$ (for dimension of $\mathcal{H}=N$ ) is called the Stiefel manifold, denoted $V_{1, N}(\mathbb{C})=U(N) / U(N-1)=\mathcal{S}\left(\mathbb{C}^{N}\right)$. It is the manifold of 1-frames in the space $\mathcal{H}=\mathbb{C}^{V}$. We will call the bundle (4.29) with the connection (4.42) the Stiefel bundle. It is the special case, $\mathcal{N}=1$, of a whole class of Stiefel bundles:

$$
\eta_{\mathcal{N}}: U(\mathcal{N}) \rightarrow V_{\mathcal{N}, N}(\mathbb{C}) \rightarrow G r_{\mathcal{N}, N}
$$

Here $G r_{\mathcal{N}, N}=U(N) / U(\mathcal{N}) \times U(N-\mathcal{N})$ is the Grassmanian manifold of $\mathcal{N}$-dimensional subspaces (or $\mathcal{N}$-dimensional projection operators) in the $N$-dimensional Hilbert space $\mathcal{H}=\mathbb{C}^{N}$ and $V_{\mathcal{N}, N}(\mathbb{C})=U(N) / U(N-\mathcal{N})$ is the Stiefel manifold of $N$-frames in $\mathcal{H}=\mathbb{C}^{N}$. The structure group of this bundle is $U(\mathcal{N})$. We will not explain the cases $\mathcal{N}>1$ any further here ${ }^{6}$ but continue with the case $\mathcal{N}=1$.

The Stiefel connection (4.42) or (4.32) is the mathematically natural definition of a connection for the bundle $\eta=\eta_{1}$. This connection is given without any reference to a Hamiltonian or an equation of motion. For a given closed curve $\mathcal{C}(\mathcal{C}$ is determined by the Hamiltonian and the dynamical equation (2.3b)) the holonomy is a purely geometric property of the Stiefel bundle; the gauge group is the structure group of this bundle.

Comparing (4.41) with (4.23) and (4.33) with (4.25) we see that the AnandanAharanov phase angle $\gamma(\mathcal{C})$ is identical with the holonomy $\hat{\gamma}$ and the A-A lift $\tilde{\psi}(t)$ is identical with the horizontal lift of the Stiefel connection:

$$
\tilde{\psi}(t)=\hat{\psi}(t) \quad ; \quad \gamma(\mathcal{C})=\hat{\gamma}
$$

Also, the non-adiabatic connection form (4.24) is the same as the Stiefel connection, $A=\mathcal{A}$. Of course, this is what we should have expected, because the consequence (4.15) of the definition (4.13) is identical with the definition (4.32b).

Thus, the Stiefel bundle is the mathematical structure that underlies the theory of the quantum geometric phase.

We started our investigation (in section 2) with a geometric phase, (2.50), which is not the holonomy of a closed path $\mathcal{C}$ of physical states. Instead we had chosen a closed path $(2.7)=(4.1)$ in another base space, the parameter space $M$ of the observables. The bundle that underlies the adiabatic geometric phase (Berry's phase) is therefore a $U(1)$ fibre bundle over the parameter space $M$ :

$$
\lambda: U(1) \rightarrow \lambda \rightarrow M
$$


We shall now discuss the relation between the bundle $\lambda$, which Simon ${ }^{7}$ recognized as the mathematics behind Berry's phase factor, and the Stiefel bundle of the AnandanAharonov approach.

As the Berry-Simon and the Anandan-Aharonov approach use different base spaces we first have to establish a relation between the base space $M$ and the base space $\mathcal{P}(\mathcal{H})$. Then we use the classification theorem for principal fibre bundles to obtain all the bundles $\lambda$ that relate to the bundle $\eta$ in (4.29). The problem is thus to find the right map:

$$
M \rightarrow \mathcal{P}(\mathcal{H})\left(=\mathbb{C} P^{N-1} \text { for } N=\text { finite }\right) .
$$

There always exists a smooth map $f_{n}$ which is determined by the Hamiltonian $h(x)$ and defined by

$$
M \ni x \rightarrow f_{n}(x)=\Lambda_{n}(x)=|n ; x><n ; x| \in \mathbb{C} P^{N-1}
$$

(for every given value of the energy quantum number $n$ ). This smooth map associates with the closed path $\mathbf{C}$ in $M$ a closed curve of (one-dimensional) eigenprojections of $h(x)$ in $\mathcal{P}(\mathcal{H})$

$$
\mathcal{C}^{\Lambda}: \Lambda_{n}(x(0)) \rightarrow \Lambda_{n}(x(t))=|n ; x(t)\rangle\langle n ; x(t)| \rightarrow \Lambda_{n}(x(T))=\Lambda_{n}(x(0))
$$

As already mentioned in section 2 (following eq. (2.33)), the closed curve (4.48) is a path of observables not of physical states. Any path of states $t \rightarrow W^{\prime}(t)$ with the initial condition $W^{\prime}(0)=\Lambda_{n}(x(0))$ where $\Lambda_{n}(x(0))$ is an eigenprojector of $h(x(0))$, is not closed. Thus the smooth map $f_{n}$ does not relate closed curves in $M$ to closed curves of physical states $\mathcal{C}$. Only in the adiabatic approximation (2.24) do the closed curves of eigenprojectors (4.48) approximate a physical state.

The Berry phase $\gamma_{n}(\mathbf{C})$ is according to (2.51) and (2.49) the holonomy of the closed curves $\mathcal{C}^{\Lambda}$ of eigenprojectors (4.48). The Berry connection form (2.42) is the Stiefel connection, however it is not taken on the closed path $\mathcal{C}$ of physical states (4.2), but on the closed path $\mathcal{C}^{\Lambda}$ of eigenprojectors $(4.48)$ in $\mathcal{P}(\mathcal{H})$ or on the closed path $\mathbf{C}$ of parameters (4.1) in $M$.

The single-valued eigenvectors $|n ; x(t)\rangle$ of $h(x(t)),(2.4)$, are sections, however they are not taken on a closed path above the cyclic path $\mathcal{C}$ of physical states (4.2) but they are taken on a closed path above $\mathcal{C}^{\Lambda}$ or $C$ in $M$. For the example (3.1) the sections $\phi_{k}(W)$ are taken above the closed path $t \rightarrow|\psi(t)\rangle\langle\psi(t)|=| k, \tilde{\theta}, \varphi(t)\rangle\langle k ; \tilde{\theta}, \varphi(t)|$ and given by (4.7): $\phi_{k}(W(t))=\mid k ; \tilde{\theta}=$ const. $\left.\varphi=\omega t\right\rangle$. But the single-valued eigenvectors of $h(R(t))$ are taken above the path $R(\theta=$ const, $\varphi=\omega t)$ of (3.53) and given by (3.11). These two sets of functions are related by the map (3.64):

$$
|k ; \tilde{\theta}, \varphi\rangle=\left|k ; F_{\nu}(\theta, \varphi)\right\rangle \quad ; \nu=\frac{\omega}{b}
$$

The Berry (adiabatic) connection on the path (3.53) in parameter space is given by

$$
A^{k}(\theta, \varphi)=i\langle k ; \vartheta, \varphi|d| k ; \theta, \varphi\rangle=-k(1-\cos \theta) d \varphi(t)
$$


and the Anandan-Aharonov connection on the same path $R(\theta, \omega t)$ in parameter space is

$$
\begin{aligned}
A^{\phi_{k}}(\theta, \varphi)=i\left\langle\phi_{k}|d| \phi_{k}\right\rangle & =i\left\langle k ; F_{\nu}(\theta, \varphi)|d| k ; F_{\nu}(\theta, \varphi)\right\rangle \\
& =-k\left(1-\frac{b}{\Omega} \cos \theta+\frac{\omega}{\Omega}\right) d \varphi
\end{aligned}
$$

which leads to the two different expressions (3.54) and (3.98) for the adiabatic and exact geometric phase connected with the same closed path $\mathbf{C}_{\boldsymbol{i}}$ in the parameter space $M$.

In addition to the $U(1)$-Stiefel bundle over the space of pure physical states (or of one-dimensional eigenprojectors $\mathcal{P}(\mathcal{H})=\mathbb{C} P^{N-1}$ ) we have thus a $U(1)$ bundle over the parameter manifold $M$ as base space.

The Stiefel bundle (4.29) is the universal classifying $U(1)$-bundle. ${ }^{5)}$ The classification theorem states that any $U(1)$ principal fibre bundle $\xi$ is isomorphic to the pull-back bundle $f^{*}(\eta)$ for some continuous function $f: M \rightarrow \mathcal{P}(\mathcal{H})$. (Appendix)

The pullback bundle $f^{*}(\eta)$ is a new bundle which one can define for each smooth map $f$ and original bundle $\eta$. It has the same fibre $F$ as the original bundle and is defined in the following way: $f^{*}(\eta)$ is the subspace of $M \times \eta=M \times \mathcal{S}(\mathcal{H})$ which consists of the points $(x, \psi) \in M \times \eta$ such that $f(x)=\pi(\psi)$. Thus

$$
f^{*}(\eta)=\left\{(x, \psi) \in M \times \mathcal{S}(\mathcal{H}) ; \Lambda_{n}(x)=|\psi><\psi|\right\}
$$

if we choose for $f$ the map $f_{n}=\Lambda_{n}$ of (4.47) and for the projection the map $\pi(|\psi\rangle)$ of (4.28). The fibre $\mathcal{F}_{x}$ of $f^{*}(\eta)$ is a copy of the fibre $\mathcal{F}_{f(x)}$ of $\eta: \mathcal{F}_{x}=\mathcal{F}_{f(x)}$ which in our case means that

$$
\mathcal{F}_{x}=\mathcal{F}_{\Lambda_{n}(x)}=\mathcal{F}_{|\psi><\psi|}=\left\{|\psi>;| \psi>=e^{i \zeta} \psi_{0}\right\} \doteq\left\{e^{i \zeta}\right\}=U(1)
$$

The projection map of the pullback bundle $f^{*}(\eta) \stackrel{\pi_{f}}{\longrightarrow} M$ is defined by $\pi_{f}:(x, \psi) \rightarrow x$. If we define $f_{*}: f^{*}(\eta) \rightarrow \eta$ by $f_{*}:(x, \psi) \rightarrow \psi$ then the pair of maps $\left(f_{*}, f\right)$ is a bundle morphism between the two bundles $f^{*}(\eta)$ and $\eta$. This means

$$
f \circ \pi_{f}=\pi \circ f_{*},
$$

or, in other words the following diagram is commutative:

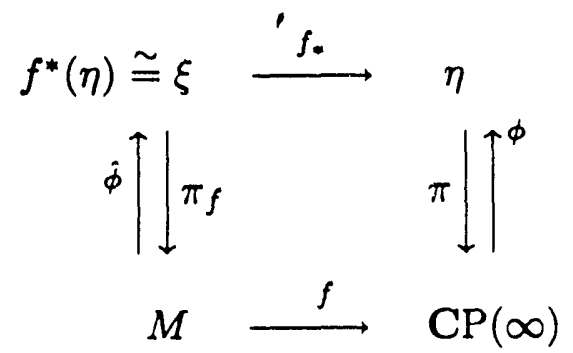

The commutativity of the diagram (4.52b) follows because

$$
\begin{aligned}
& f \circ \pi_{f}(x, \psi)=f(x)=\Lambda_{n}(x)=|\psi\rangle\langle\psi| \\
& \pi \circ f_{*}(x, \psi)=\pi(\psi)=|\psi\rangle\langle\psi|
\end{aligned}
$$


Sections pull back, too. Thus if $\phi: \mathcal{P}(\mathcal{H}) \rightarrow \eta$ is a section of $\eta$ then the pull-back section $\hat{\phi}: M \rightarrow f^{*}(\eta)$ is defined by

$$
\hat{\phi}(x) \equiv\left(x, \phi\left(\Lambda_{n}\right)\right)=(x, \phi(f(x))=|n ; x\rangle
$$

The diagram (4.52) with the sections is also commutative in the sense

$$
f_{*} \circ \hat{\phi}=\phi \circ f
$$

This commutativity follows since

$$
f_{*} \circ \hat{\phi}(x)=f_{*}\left(x, \phi\left(\Lambda_{n}\right)\right)=\phi\left(\Lambda_{n}\right)=\phi(f(x))
$$

Two homotopic maps $f_{1}: n \rightarrow \mathcal{P}(\mathcal{H})$ and $f_{2}: M \rightarrow \mathcal{P}(\mathcal{H})$ (Appendix) lead to isomorphic bundles $f_{1}^{*}(\eta)$ and $f_{2}^{*}(\eta)$.

Thus there is a one-to-one correspondence between $U(1)$ bundles over $M$ and the homotopy classes of maps of $f: M \rightarrow \mathbb{C} P(\infty)$. For every homotopy class $[f]$ of maps there exist one $U(1)$ bundle $f^{*}(\eta)$ (up to equivalence) that is determined by this map $[f]$.

In the construction of the pullback bundle in (4.52) we used the map $f$ of (4.47) given by the eigenprojectors $\Lambda_{n}$ of the Hamiltonian. From our example (3.1) we know that the closed path of eigenprojectors $f=\Lambda_{k}(\theta, \varphi)=|k ; \theta, \varphi\rangle\langle k ; \theta, \varphi| \in \mathcal{P}(\mathcal{H})$ which is the image under $f$ of the closed path $t \rightarrow R(\theta, \omega t)$ in $M$ is not a closed path of physical states. Thus the $|\psi\rangle\langle\psi|$ and $\psi$ in (4.52a) are not solutions of the dynamical equations $(2.3 \mathrm{~b})$ and $(2.3 \mathrm{a})$ respectively. From the results $(2.32)$ we know tht this is generally true. The map (4.47) does not map closed paths in $M$ into closed paths of physical states. We thus have to find in place of the map $f$ another map $\tilde{f}: M \rightarrow \mathcal{P}(\mathcal{H})$ that maps closed paths in the parameter space $M$ into closed paths of physical states (solutions of $(2.3 \mathrm{~b})$ ) in $\mathcal{P}(\mathcal{H})$. To find such maps we will use our example (3.1) as a guide. The closed path of physical states that corresponds to the closed path $t \rightarrow R(\theta, \omega t)$ in parameter space is given by

$$
\begin{aligned}
t \rightarrow|k ; \tilde{\theta}, \omega t\rangle\langle k ; \tilde{\theta}, \omega t| & =\left|k ; F_{\nu}(\theta, \omega t)\right\rangle\left\langle k ; F_{\nu}(\theta, \omega t)\right| \\
& \equiv \tilde{\Lambda}_{k}(\theta, \omega t)
\end{aligned}
$$

where

$$
|k ; \tilde{\theta}, \varphi\rangle \equiv e^{-i \varphi J_{3}} e^{-i \tilde{\theta} J_{2}} e^{i \varphi J_{3}}\left|k ; \vec{e}_{3}\right\rangle=\left|k ; F_{\nu}(\theta, \varphi)\right\rangle
$$

are the sections of (3.84). ( $F_{\nu}$ and $\tilde{\theta}$ are given by (3.66) and (3.62) respectively). The projection operator $\bar{\Lambda}_{k}(\theta, \omega t)$ is no more an eigenprojector of the time-dependent Hamiltonian $h(R(\theta, \omega t))$, it is the eigenprojector of an other operator defined by

$$
\tilde{h}(\theta, \varphi)=h(\tilde{\theta}, \tilde{\varphi})=h\left(F_{\nu}(\theta, \varphi)\right)=b \vec{R}(\tilde{\theta}, \varphi) \cdot \vec{J}
$$

However this projection operator $\tilde{\Lambda}_{k}$ is a cyclically evolving state (solution of the dynamical equation (2.3)):

$$
\tilde{\Lambda}_{k}(\theta, \omega t)=|\psi(t)\rangle\langle\psi(t)|=W(t)
$$


where $\psi(t)$ is the solution (3.99) of the equation (3.56). Therefore we choose in place of the map $f$ in the diagram (4.52) the map

$$
\tilde{f}_{k}=f_{k} \circ F_{\nu}: \mathcal{S}^{2} \ni R \rightarrow \tilde{\Lambda}_{k}(R) \in \mathbb{C} P(\infty), \quad \nu<1
$$

This map $\tilde{f}_{k}$ associates with every $\mathbf{R}(\theta, \varphi) \in \mathcal{S}^{2}$ an eigenstate $\tilde{\Lambda}_{k}$ of the operator $\mathbf{R}(\tilde{\theta}, \varphi) \cdot \vec{J}$ with eigenvalue $k$ ( $k$ being the component of angular momentum along $\mathbf{R}(\tilde{\theta}, \varphi)$ not along the direction of the magnetic field $\mathbf{R}(\theta, \varphi)$. The restriction $\nu<1$ is necessary so that $F_{\nu}$ and therewith $\tilde{f}_{k}$ is continuous.

We take this example as our guiding principle for the general case: There exist classes of time-dependent periodic Hamiltonians for which the dynamical equation (2.3) has cyclic non-degenerate solutions with the same period as the period $\omega$ of the Hamiltonian. ${ }^{8}$ ) (We want to restrict ourselves here to the case of non-degenerate solutions, in the case of an $\mathcal{N}$-fold degeneracy the $U(1)$ principal bundle has to be replaced by the Stiefel bundle with structure group $\left.U(\mathcal{N}) .^{6}\right)$ ) These classes are characterized by the existence of a homeomorphism (diffeomorphism)

$$
F: M \rightarrow M
$$

which has the following properties:

1.) The operator $\tilde{h}(x)$ defined by

$$
\tilde{h}(x) \equiv h(F(x)) \quad \text { has eigenprojectors } \quad \tilde{\Lambda}_{n}(x)=\Lambda_{n}(F(x))
$$

For a closed path (4.1) these eigenprojectors are cyclic solutions of (2.3b): $\Lambda_{n}(F(x(t))=W(t)$.

2.) $F$ is continuously related to the identity $F(x, \nu) \rightarrow x$ for $\nu \rightarrow 0$, where $\nu$ are parameters of the Hamiltonian $h(x(t))=h_{\nu}(x(t))$ with $\left[h_{\nu=0}(t), h_{\nu=0}\left(t^{\prime}\right)\right]=0$ for $t \neq t^{\prime}$

In our example (3.1), the parameter is given by $\nu=\omega / b$ where $\omega$ is the precession frequency and $b$ is the Larmor frequency.

The map

$$
\tilde{f}=f \circ F: M \ni x \rightarrow \tilde{\Lambda}_{n}(x) \in \mathbb{C} P(\infty)=\mathcal{P}(\mathcal{H})
$$

where $f$ is given by (4.47), associates to every $x \ni M$ an eigenstate $\tilde{\Lambda}_{n}(x) \in \mathcal{P}(\mathcal{H})$ of $\tilde{h}(x)$. This map $\tilde{f}$ also associates to a closed curve (4.1) in $M$ a closed curve of states $W(t)=\tilde{\Lambda}(x(t))$ in $\mathcal{P}(\mathcal{H})$. With this map $\tilde{f}$ we construct the pullback bundle over the parameter space using the classifying theorem of $U(1)$ principal fibre bundles. The classifying theorem of fibre bundles thus becomes the central mathematical tool in establishing the relation between the Stiefel bundle of the Anandan-Aharonov approach and the parameter space $U(1)$ bundle over $M$ in the Berry-Simon approach. 
The $U(1)$ bundle over $M$, that associates closed paths in $M(4.1)$ with closed paths of physical staies (4.2) is given by the pullback $\tilde{f}^{*}$ of $(4.60)$ :

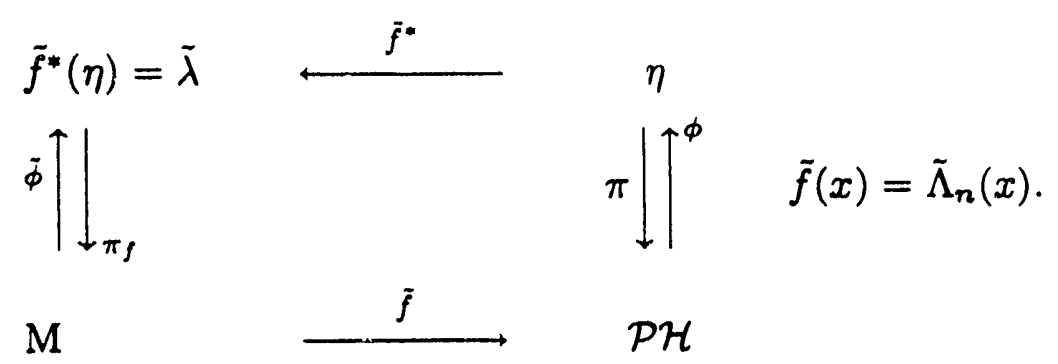

Thus for every value $n$ we obtain a map $\tilde{f}$ and a $U(1)$ bundle over parameter space $\tilde{\lambda}$

To see how Berry connection and Berry phase are obtained, we start with the universal classifying bundle $\eta$ of (4.29). The bundle $\eta$ carries as a natural connection the Stiefel connection. We choose a (local) section $|\phi(\tilde{\Lambda})\rangle$ of $\mathcal{P}(\mathcal{H}) \supset O \rightarrow S(\mathcal{H})$ with the property $|\phi(\tilde{\Lambda})\rangle\langle\phi(\tilde{\Lambda})|=\tilde{\Lambda}_{n}$. The Stiefel connection is then (locally) represented by the $U(1)$-valued one-form

$$
\mathcal{A} \equiv i\langle\phi|d| \phi\rangle
$$

The local section $\tilde{\phi}$ in the fibre bundle $\tilde{f}^{*}(\eta)$ is given by

$$
\tilde{\phi}(x)=\phi(\tilde{f}(x)=\phi(f(F(x)))=|n ; F(x)\rangle
$$

The $|n ; \tilde{x}\rangle=|n ; F(x)\rangle$ are eigenvectors of $\tilde{h}(x)$ and are determined up to a gauge transformation (4.6).

The canonical connection one-form on the bundle $\tilde{\lambda}=\tilde{f}^{*}(\eta)$ is obtained from (4.62):

$$
A(x) \equiv \tilde{f}^{*} \mathcal{A}=\left\langle n ; F(x)\left|\frac{\partial}{\partial x^{\mu}}\right| n ; F(x)\right\rangle d x^{\mu}
$$

The geometric phase factor (holonomy) is then given by

$$
\begin{aligned}
\exp i \gamma(\mathcal{C}):=\exp i \oint_{\mathcal{C}=\tilde{f} C} \mathcal{A} & =\exp \left(-\oint_{\mathcal{C}=\tilde{f} C}\left\langle\phi\left|d_{i}\right| \phi\right\rangle\right) \\
& =\exp i \oint_{C} \tilde{f}^{*} \mathcal{A}=\exp \left(-\oint_{C}\left\langle n ; F(x)\left|\frac{\partial}{\partial x^{\mu}}\right| n ; F(x)\right\rangle d x^{\mu}\right) .
\end{aligned}
$$

The path in the first two integral is over the closed curve $(4.2)$ in $\mathcal{P}(\mathcal{H}), \mathcal{C}=\tilde{f} \circ C$. The path in the second two integral $C$ is the closed curve (4.1) in $M$. In the third equality the property of the pullback was used. This formula (4.65) says that the geometric phase can be either calculated using the canonical (Stiefel) connection $\mathcal{A}$ in the universal bundle $\eta$ or using its pullback $\tilde{f}^{*} \mathcal{A}$ in the induced bundle. This means the geometric phase factor $e^{i \gamma(\mathcal{C})}$ (the holonomy) acquired after parallel transport with respect to the Stiefel connection along the closed curve $\mathcal{C}$ in $\mathcal{P}(\mathcal{H})$ is the same as the geometric phase factor acquired after parallel transport with respect to $A(x) \equiv \tilde{f}^{*} \mathcal{A}$ along the closed curve $C$ in the parameter space $M$.

The exr ression in terms of the integral over $\mathcal{C}$ in $\mathcal{P}(\mathcal{H})=\mathbf{C} P(\infty)$ is the standard expression for the Aharonov-Anandan phase. Thus tha Aharonov-Anandon connections and therewith the geometric phase can also be obtained from the eigenvectors of the 
Hamiltonian, if $F$ is known. However this phase was introduced originally not through the Stiefel connection but as the difference between the total pha' 9 and the dynamical phase defined by $\exp (-i \oint\langle\psi(t)|h(t)| \psi(t)\rangle d t)$.

The Berry connection and phase is obtained from (4.64) and (4.65) in the adiabatic approximation in which $F$ is replaced by the identity

$$
\begin{aligned}
A & =\left\langle n ; x\left|\frac{\partial}{\partial x^{\mu}}\right| n ; x\right\rangle d x^{\mu} \\
e^{i \gamma(C)} & =\exp \left(-\oint_{C}\left\langle n ; x\left|\frac{\partial}{\partial x^{\mu}}\right| n ; x\right\rangle d x^{\mu}\right)
\end{aligned}
$$

where $C$ is the closed curve (4.1) which also appears in (4.45).

The B-S bundle (4.45) $\lambda=f^{*}(\eta)$ and the bundle $\tilde{\lambda}=\tilde{f}^{*}(\eta)$ of (4.61) are related by the bundle morphism $F^{*}$ :

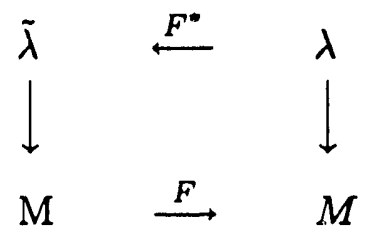

As $F$ is a diffeomorphism continuously related to the identity, the two maps $f=\tilde{f} \circ F^{-1}$ and $\tilde{f}$ belong to the same homotopy class in $[M, \mathcal{C} P(\infty)]$. Consequently $\lambda, \tilde{\lambda}$ have the same topology. ${ }^{9)}$

In this section 4 we gave a general description of the structures which we discovered in section 3 for the specific example given by (3.1). We introduced the geometric phase for general cyclic (non-adiabatic) evolution and showed that the adiabatic phase introduced in section 2 is a limit of the general geometric phase. But we also showed that exact cyclic adiabatic evolution is always stationary, i.e. the closed path is a point in $\mathcal{P}(\mathcal{H})$.

We uncovered the mathematical structure behind the gauge theory of the geometric phase and showed that this involved two fibre bundles with the structure group $U(1)$ : the $U(1)$ bundle over the parameter space $M$ (differentiable manifold) of the parameter dependent Hamiltonian in the adiabatic approximation considered by Berry and Simon and the $U(1)$ Stiefel bundle in the Anandan Aharonov approach where the base space is the space of (pure) physical states $\mathcal{P}(\mathcal{H})=\mathbb{C} P(\infty)$.

We then related the time evolution (4.2) of a cyclic quantum state $W(t)$ (considered in the Aharanov-Anandan approach) to the change of a parameter dependent Hamiltonian and its eigenprojectors along a closed path (4.1) in parameter space, (considered in the B-S approach). Since the Stiefel bundle is the universal classifying bundle of the $U(1)$ bundles over manifolds $M$, the classifying theorem provides all $U(1)$ bundles over $M$ (characterized by the first Chern class). Bundles and Chern class are labeled by the quantum number $n$.

The classifying Stiefel bundle has a natural connection (4.42) given by differential geometry (Stiefel connection) which is identical to the connection obtained from the Aharanov-Anandan approach by separating from the total phase in a natural way a dynamical phase. The connection in the parameter space bundle for exact (non-adiabatic) evolution is the pullback (4.64) of the Stiefel connection (4.62). This pullback connection goes into Berry's connection (4.66) in the adiabatic limit. 
The mathematics of the fibre bundles was created and the classification theorem of principal bundles was established ${ }^{5)}$ long before the quantum geometric phase was considered to be of any importance in physics. Though we have accepted the fact that physics is described by mathematical structures, one usually faces the situation that the mathematics is not quite ready for sorne new physical theory and has to be created along with, or adapted specifically for, this new physics. It is very rare that the mathematical structure is already all there and the physical quantities need just to be mapped upon the elements of this mathematical structure. This was the situation for the quantum geometric phase and was therefore the cause of great excitement and exultation. The role which the mathematics of fibre bundles plays for the physics connected with the quantal geometric phase is one of the more spectacular examples of what Wigner calls "the unreasonable effectiveness of mathematics in natural science." 


\section{References}

1.) Y. Aharonov and J. Anandan, Phys. Rev. Lett. 58, 1593 (1987); J. Anandan and Y. Aharonov, Phys. Rev. D38, 1863 (1988).

2.) The method used in section 3 can be generalized in a straight forward way for Hamiltonians that are linear functions of generators of any Lie algebra. For more details and for other calculational methods see reference 8 below.

3.) The generalization to $U(\mathcal{N})$ bundles describing the non-abelian gauge theory for $\mathcal{N}$-fold degenerate states is straightforward and causes few new principal problems; cf. reference 8 of section 1 .

4.) More precisely, it is a dense subspace of $\mathcal{H}$ for $N \rightarrow \infty$, reference 12 of section 1. The projective space $\mathbb{C} P(\infty)$ is the inductive limit of the $\mathbb{C} P^{N}$ for $N \rightarrow \infty$. To avoid all questions connected with $N \rightarrow \infty$ we want to assume here that $N$ is arbitrary large but finite. For the infinite dimensional case we shall always assume that we are in one particular $\mathbb{C} P^{N-1}$ with $N$ finite. This is always possible if the Hamiltonian is in the enveloping algebra of a compact group of which (3.1) is the simplest example.

5.) What we call Stiefel connection here is usually called the universal connection because the bundle (4.29) is the universal classifying bundle. M. Narasinhan and S. Ramanan, Amer. J. Math. 83, 563 (1961). See also Ch. J. Ishan. Modern Differential Geometry for Physicists, World Scientific Publ. (1989) section 3.2.

6.) The non-abelian case $\mathcal{N}>1$ is discussed in reference 8 of section 1 .

7.) Reference 11 of section 1.

8.) D.J. Moore, Phys. Rep. 240, No. 1 (1992) sect. 3,4 and 5.

9.) For our example (3.1) the map $F=F_{\nu}(x)$ of (3.66) becomes discontinuous for $\nu \geq 1$. As a consequence the bundles $\tilde{\lambda}$ and $\lambda$ are no $m$ ore isomorphic for $\nu>1$. For details see: A Mostafazadeh, A. Bohm. Topological Aspects of the Non-Adiabatic Berry Phase. 


\section{Acknowledgement:}

I should like to express my gratitude to my friends and colleagues who taught me and worked with me on this subject: Ali Mostafazadeh, Mark Loewe, L.J. Boya, B. Kendrick, J. Lemke and G. Rudolph. I am grateful to M.V. Berry, C.A. Mead and J. Anandan who read parts of earlier stages of the manuscript and made suggestions and gave me advice. This paper is dedicated to W. Drechsler on the occasion of his coming birthday, who was the first to teach me about fibre bundles. 


\title{
Mathematical Appendix:
}

\author{
"A Brief Review of Fibre Bundles and Their Classification" \\ by
}

\section{Ali Mostafazadeh}

\section{A.I. Fibre Bundles:}

Definition: A fibre bundle ${ }^{(1),(4)}$ is a collection $(E, M, \pi, G)$ of two (smooth) manifolds $E$ and $M$, an onto continuous (smooth) map $\pi: E \longrightarrow M$ and a Lie group $G \cdot E, \eta, \pi$, and $G$ are called the "total space," the "base manifold," the "projection map" and the "structure group", respectively. For any $x \in M$, the set $\pi^{-1}(x)$ is a (smooth) manifold and $\pi^{-1}(x)=: F_{x}$ is called the fibre over $x$. As a point set $E=\bigcup_{x \in M} F_{x}$. Furthermore, all fibres look alike, i.e. there exist a (smooth) manifold $F$ such that $F_{x}$ are homeomorphic (diffeomorphic) to $F$. $F$ is called the "typical fibre". The structure group $G$ is a group of diffeomorphisms of $F$ and it has a global right (or left) action on $E$. The collection $(E, M, \pi, G)$, which sometimes collectively called $E$, is said to have a fibre bundle structure if for any $x \in M$ there exist an open neighborhood $O_{i}$ of $x$ such that

$$
\pi^{-1}\left(O_{i}\right) \simeq O_{i} \times F
$$

Moreover, the (right) action of $G$ on $E$ is such that $\forall p \in E, \forall g \in G$ :

$$
\pi(p . g)=\pi(p)
$$

i.e. the action of $G$ moves the points along the fibres. Furthermore, $G$ acts on fibres freely and transitively.

Definition: If $F$ happens to be identical to $G$ (as manifolds), then the fibre bundle is called a "principal fibre bundle".

Definition: If $E=\pi^{-1}(M) \simeq M \times F$ then the fibre bundle is said to be "trivial" or a "product" bundle. Not every fibre bundle is trivial. The degree in which a fibre bundle differs from being trivial is measured by a set of functions called the "transition functions" $\left\{g_{i j}\right\}$. Let $\left\{O_{i}\right\}$ be on open covering of $M=\bigcup_{i} O_{i}$ such that

$$
\varphi_{i}: \pi^{-1}\left(O_{i}\right) \rightarrow O_{i} \times F
$$

are the diffeomorphisms of (A.1). Then one can view $E$ as a collection of patches $O_{i} \times F$ over $O_{i}$. The global or topological structure of $E$ is characterized by gluing these patches in their intersections. Let $O_{i}$ and $O_{j}$ have a nonempty intersection then the gluing map is defined by:

$$
\begin{aligned}
g_{i j}:= & \varphi_{j} \circ \varphi_{i}^{-1}: \varphi_{i}\left(O_{i} \cap O_{j}\right) \longrightarrow \varphi_{j}\left(O_{i} \cap O_{j}\right) \\
& : \quad\left(O_{i} \cap O_{j}\right) \times F \longrightarrow\left(O_{i} \cap O_{j}\right) \times F
\end{aligned}
$$

Then $\forall x \in O_{i} \cap O_{j}$ 


$$
\begin{aligned}
g_{i j}(x) & :\{x\} \times F \rightarrow\{x\} \times F \\
& : F \longrightarrow F
\end{aligned}
$$

and

$$
g_{i j}(x) \in G .
$$

Example: A nontrivial and quite relevant ${ }^{(2)}$ example of a principal bundle is the Hopf bundle: $\left(S^{3}, S^{2}, \pi, U(1)\right)$ also denoted by:

$$
S^{1}=U(1) \longrightarrow S^{3}=S U(2) \longrightarrow S^{2}=S U(2) / U(1) .
$$

where the fibres are $U(1)$ orbits in $S U(2)^{(3)}$.

\section{A.II: Sections, Lifts, Connections and Holonomy:}

Let $(E, M, \pi, G)$ be a fibre bundle.

Definition: For any open submanifold $O \subset M$, a continuous (smooth) map $S$ : $O \rightarrow E$ is called a "section" over $O$ if

$$
\pi \circ S=\left.i d\right|_{O}
$$

If $O=M$ then $S$ is said to be a global section of $E$.

Remark: A principal bundle is trivial if and only if it has a global section.

Definition: Let $\mathbf{C}:[0, T] \rightarrow M$ be a continuous (smooth) curve in $M$. Then a curve $C:[0, T] \rightarrow E$ in $E$ is said to be a lift of $\mathbf{C}$ if

$$
\pi \circ C=\mathbf{C}
$$

If a principal bundle $P$ is endowed with a connection (a geometric structure), one has a well-defined notion of parallel transport. ${ }^{(4)}$ Then, a point $p_{0} \in P$ can be parallelly transported along the curve $\mathbf{C}$, to define a curve

$$
C: p_{0} \longrightarrow p(t) \rightarrow p(T)
$$

in $P$ which is called the "horizontal lift" of $\mathbf{C}$ associated with the connection on $P$.

Alternatively, a connection on $P$ may be defined in terms of horizontal lifts. More precisely, a connection is realized as a rule which associates a lift $C$ to any pair $\left(\mathbf{C}, p_{0}\right)$. The tangent vectors $w_{t}:=\left.\frac{d}{d s} C(s)\right|_{s=t}$ to $C(t)$ which project to the tangent vectors $v_{t}$ to $\mathbf{C}(t)$ are called "horizontal vectors". Note that $w_{t} \in T_{C(t)} P$, and $v_{t} \in T_{\mathrm{C}(t)} M$ where $T_{C(t)} P$ denotes the tangent space of the bundle $P$ at $C(t)$ and $T_{\mathbf{C}(t)} M$ denotes the tangent space of the base space $M$ at the point $\mathbf{C}(t)$. Since one can reconstruct $C$ from the knowledge of the horizontal vectors, one has ${ }^{(4)}$ : 
Definition (1): A connection on $(P, M, \pi, G)$ is a linear mapping $\sigma_{p}: T_{x} M \rightarrow$ $T_{p} P, \forall_{p} \in P$, such that:

i) $\pi^{\prime} \circ \sigma_{p}: T_{x} M \rightarrow T_{x} M$ is the identity map.

ii) $\sigma_{p}$ depends smoothly on $p \in P$.

iii) $\sigma_{p \cdot g}=R_{g}^{\prime} \sigma_{p}$

where, $\pi^{\prime}: T_{p} P \rightarrow T_{\pi(p)} M$ is the push forward map corresponding to $\pi: P \rightarrow M$, and $R_{g}^{\prime}: T_{p} P \rightarrow T_{p} P$ is the push forward map corresponding to the right action map of $g \in G, R g: P \rightarrow P$, which is denoted by $R_{g}(p)=p \cdot g, \forall_{p} \in P$ and $g \in G$. The right action of $G$ on $P$ is defined as follows: Let $g \in G, x \in O_{i} \subset M, p \in \pi^{-1}(x) \subset P$, and $\varphi_{i}(x):\{x\} \times \pi^{-1}(x) \rightarrow G$ be the restriction of $\varphi_{i}: \pi^{-1}\left(O_{i}\right) \rightarrow O_{i} \times F$ (of A.I) onto the fibre over $x, \pi^{-1}(x)$. Clearly, $\varphi_{i}(x)$ is a diffeomorphism identifying the fibre $\pi^{-1}(x)$ with $G$. The right action map $R_{g}: P \rightarrow P$ is defined by

$$
R_{g}(p)=p \cdot g:=\varphi_{i}^{-1}\left[\varphi_{i}(p) \cdot g\right]
$$

where $\varphi_{i}(p) \in G$ and the product in the bracket is the group product.

The horizontal vectors span a subspace of $T_{p} P, \forall_{p} \in P$ which is called the "horizontal subspace $H_{p} P$ of $T_{p} P$. Hence, a connection leads to a, not necessarily orthogonal, decomposition of $T_{p} P$ into:

$$
T_{p} P=H_{p} P+V_{p} P
$$

$V_{p} P$ is called the "vertical subspace". One can view a connection as a rule which determines $H_{p} P, \forall_{p} \in P$ :

Definition (2): A connection on $(P, M, \pi, G)$ is a collection of vector spaces $H_{p} P \subset$ $T_{p} P$, such that

i) $\left.\pi\right|_{H_{p} P}: H_{p} P \rightarrow T_{\pi(p)} M$ is an isomorphism.

ii) $H_{p}$ depends smoothly on $p \in P$.

iii) $H_{p \cdot g}=R_{g}^{\prime} H_{p}$.

The vertical subspaces $V_{p} P$ are actually canonically isomorphic to the Lie algebra $\mathcal{G}$ of $G$. This is seen as follows: For all $X=\left.\frac{d g_{(s)}}{d s}\right|_{s=0} \in T_{e} G=\mathcal{G}$, let $v(p) \in V_{p} P$ be defined by

$$
v(p):=\left.\frac{d(p \cdot g(s))}{d s}\right|_{s=0}
$$

Then, (A.2) establishes an isomorphism between $\mathcal{G}$ and $V_{p} P$. This isomorphism allows one to have a more practical definition of a connection on a principal bundle.

Definition (3): A connection on $(P, M, \pi, G)$ is a 1 -form $\omega$ on $P$ with values in $\mathcal{G}$, such that

i) $\forall v_{p} \in v_{p} P, \omega_{p}\left(V_{p}\right)=X$ where $X \in \mathcal{G}$ is related to $V_{p}$ as dictated by (A.2).

ii) $\omega_{p}$ depends smoothly on $p \in P$.

iii) $\omega_{p \cdot g}\left(R_{g}^{\prime} v_{p}\right)=A d\left(g^{-1}\right) \omega_{p}\left(v_{p}\right)$ where $A d$ denotes the adjoint representation of $G$ on $\mathcal{G}$. Given $\omega$ the horizontal subspaces are obtained by

$$
H_{p}:=\left\{w_{p} \in T_{p} P: \omega_{p}\left(w_{p}\right)=0\right\}
$$


The three definitions (1), (2), and (3) can be shown to be equivalent. ${ }^{(4)}$ In the physics literature one usually encounters a 1 -form $A$ on $M$ with values in $\mathcal{G}$. This is obtained by pulling back the connection one-form $\omega$ on $M$, via a local section

$$
S_{i}: O_{i} \longrightarrow P
$$

Then, locally

$$
A_{(i)}:=S_{i}^{*}(\omega)
$$

where the subscript $i$ refers to the local patch over $O_{i} \subset M$ and $S_{i}^{*}$ denotes the pullback. If $P$ is a trivial bundle, as is the case in many gauge theories, $S_{i}$ can be taken to be a global section, i.e. $O_{i}=M$.

It turns out that the 1 -form $A_{(i)}$ is well-defined if one requires a particular transformation rule which reads:

$$
A_{(i)}=g_{i j}^{-1}(x) \cdot A_{(j)} \cdot g_{i j}(x)+g_{i j}^{-1}(x) \cdot g_{i j}^{\prime}(x)
$$

In (A.3), $g_{i j}(x) \in G$ is the transition function connecting the patch $O_{i}$ to $O_{j}, g_{i j(x)}^{-1}$ is its (group) inverse, and $g_{i j}^{\prime}(x)$ is the push forward map: $g_{i j}^{\prime}(x): T_{x} M \rightarrow \mathcal{G}$, corresponding to $g_{i j}: M \rightarrow G$. Here we have assumed $G$ to be a matrix gxoup for simplicity. The second term in the right hand side of (A.3) is known as the pullback of "MaurerCartran" 1 -form on $G$ defined by $g_{i j}: M \rightarrow G$. Another notation for $g_{i j}^{\prime}(x)$ is $d g_{i j}(x)$. To motivate this notation, let $\frac{d}{d S} \in T M_{x(s)}$ and act $g_{i j}^{\prime}(x(s))$ on $\frac{d}{d s}$. By definition of push forward map ${ }^{(1,4)}$ one has:

$$
g_{i j}^{\prime}(x(s)) \frac{d}{d s}=\frac{d}{d s}\left(g_{i j}(x(s))\right)
$$

In a local coordinate frame $\left(x^{\mu}\right)$ this becomes

$$
g_{i j}^{\prime}(x(s))\left(\frac{d x^{\mu}(s)}{d s} \frac{\partial}{\partial x^{\mu}}\right)=\frac{d x^{\mu}(s)}{d s} \frac{\partial}{\partial x^{\mu}}\left(g_{i j}(x(s))\right.
$$

which together with the fact that $g_{i j}^{\prime}(x)$ is a linear map suffices to see

$$
g_{i j}^{\prime}(x)\left(\frac{\partial}{\partial x^{\mu}}\right)=\frac{\partial}{\partial x^{\mu}}\left(g_{i j}(x)\right)
$$

Hence, in general one has:

$$
g_{i j}^{\prime}(x)=\frac{\partial}{\partial x^{\mu}} g_{i j}(x) d x^{\mu}=d g_{i j}(x)
$$

and (A.3) is then written as:

$$
A_{(i)}=g_{i j}^{-1}(x) A_{(j)} g_{i j}(x)_{+} g_{i j}^{-1}(x) d g_{i j}(x)
$$

It is remarkable that $\left(\mathrm{A} .3^{\prime}\right)$ coincides with the transformation rule for the gauge potential of particle physics.

Corisider a closed curve, a loop, $\mathbf{C}:[0, T] \rightarrow M$ in $M$ and let $p_{\circ} \in \pi^{-1}(\mathbf{C}(0))$ and $C$ be the horizontal lift associated to a connection 1-form $\omega$ on $P$. Then, since 
$\mathbf{C}(0)=\mathbf{C}(T), p(T):=C(T) \in \pi^{-1}(\mathbf{C}(T))=\pi^{-1}(\mathbf{C}(0))$, i.e. $p(T)$ belongs to the same fibre as $p_{\circ}$ does. On the other hand, $G$ has a transitive action on the fibres, thus, there exists a $g \in G$, such that

$$
p(T)=p_{\circ} \cdot g=p(0) \cdot g
$$

since $p(T)$ is the parallel transport of $p(0), g$ is determined by the one-form $A=$ $A_{\mu} d x^{\mu}=A_{\mu}^{a} d x^{\mu} \otimes J_{a}$, as:

$$
g=\mathbb{P} \exp \left[-\oint_{\mathbf{C}} A\right]=\mathcal{T} \exp \left[-\int_{0}^{T} A_{\mu}(x(t)) \frac{d^{\mu} x(t)}{d t} d t\right]
$$

where $J_{a}$ are generators of $\mathcal{G}$, and $\mathbb{P}$ and $\mathcal{T}$ stand for the path ordered and time ordered products, respectively. ${ }^{(1)}$

Definition: The set of all elements $g$ of $G$ given by (A.4), for fixed $p_{0}$, form a subgroup of $G$ which is called the "holonomy" group of $\omega$ associated to $p_{\circ} \cdot g$ is called a holonomy element.

As is seen from (A.3), $A$ does not transform as a tensor. However, one can introduce a tensorial quantity for a given connection. This is called the curvature two-form $\Omega$ of the connection $\omega$. $\Omega$ is pulled back on $M$ by a local section to define a Lie algebra valued two-form $F$ on $M$. The latter is in perfect analogy to the field strength associated to a gauge potential. $F$ is related to $A$ by the following local expression:

$$
F_{(i)}=d A_{(i)}+\frac{1}{2}\left[A_{(i)}, A_{(i)}\right]
$$

$F_{(i)}$ has the following tensorial transformation rule:

$$
F_{(i)}=g_{i j}^{-1}(x) \cdot F_{(j)} \cdot g_{i j}(x) .
$$

Finally (A.4) is also written in terms of $F$ :

$$
g=\mathbb{P} \exp \left[-\int_{S} F\right]
$$

where Stokes theorem is used and $S$ is any two-surface in $M$ bounded by C.

\section{A. III.Classification Theorem for Principal Fibre Bundles:}

Definition: Let $(\tilde{E}, \tilde{M}, \pi, G)$ be a fibre bundle, and $M$ a (smooth) manifold. Let $f: M \rightarrow \tilde{M}$ be a continuous (smooth) map of manifolds. Then one can induce a (G-) bundle structure over $M$ using $f$.

For any $x \in M$, the fibre $F_{x}$ is defined to be $\pi^{-1}(f(x))$. The "pullback bundle" is

$$
f^{*}(\tilde{E}):=\cup_{x \in M} \pi^{-1}(f(x)) .
$$

as a point set. The bundle structure is then fixed by requiring the transition functions $g_{i j}(x)$ of $E=f^{*}(\tilde{E})$ to be given by

$$
g_{i j}(x)=\tilde{g}_{i j}(f(x)) \quad \forall x \in M
$$


where $\left\{\tilde{g}_{i j}\right\}$ are transition functions of $\bar{E}$ corresponding to an open covering $\left\{\tilde{O}_{i}\right\}$ of $\tilde{M}$, and $\left\{g_{i j}\right\}$ correspond to the open covering $\left\{f^{-1}\left(\tilde{O}_{i}\right)\right\}$ of $M$.

Definition: Let $M$ and $\tilde{M}$ be two topological spaces (smooth manifolds), and $f_{1}$ and $f_{2}$ two continuous maps

$$
f_{i}: M \rightarrow \tilde{M} \quad i=1,2
$$

$f_{1}$ and $f_{2}$ are said to be "homotopic" if there exists a continuous map:

$$
\mathcal{F}: M \times[0,1] \rightarrow \tilde{M}
$$

such that $\left.\mathcal{F}\right|_{M \times\{0\}}=f_{1}$ and $\left.\mathcal{F}\right|_{M \times\{1\}}=f_{2}$. The existence of $\mathcal{F}$ is equivalent to the statement that $f_{1}$ can be continuously deformed into $f_{2}$ and vice versa.

Theorem: For any Lie group $G$, there exist a principal $G$-bundle $\eta:(\eta(G), B G, \tilde{\pi}, G)$ such that any principal $G$-bundle $(P, M, \pi, G)$ is obtained from $\eta$ as a pullback bundle i.e. there exist a continuous (smooth) map $f: M \rightarrow B G$ such that

$$
P \simeq f^{*}(\eta(G))
$$

Furthermore, homotopic maps pullback (topologically) identical bundles. ${ }^{(5)}$

The statement of the classification theorem can be illustrated by a commutative diagram:

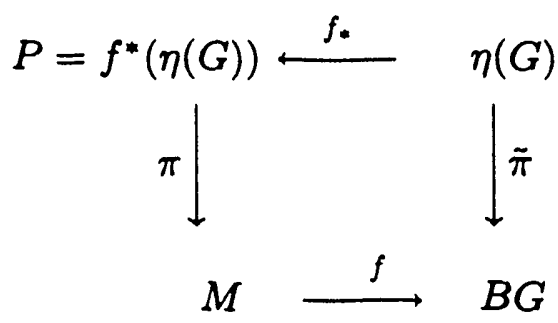

$B G$ and $\eta(G)$ are called the "classifying space" and the "classifying bundle", respectively.

For $\mathrm{G}=\mathrm{U}(\mathrm{N}), \quad B G$ can be chosen to be the complex Grassmannian $G r_{\mathcal{N}}=\bigcup_{n=1}^{\infty} G r(n, \mathcal{N})$ and $\eta$ is the so-called "universal (Stiefel) bundle." For $G=U(1), B G=G r_{1}=\mathbb{C} P(\infty)$.

Theorem: There exists a "universal" (Stiefel) connection on $\eta$ such that any connection on any principal $G$-bundle $(P, M, \pi, G)$ can be obtained as a pullback connection one-form from the Stiefel connection. ${ }^{(6)}$ 
A. IV. References used in Appendix:

(1) M. Nakahara, "Geometry, Topology and Physics," Adam Hilger, Bristol (1991).

(2) A. Bohm, L.J. Boya, A. Mostafazadeh, and G. Rudolph, "Classification Theorem for Principal Fibre Bundles, Berry's Phase, and Exact Cyclic Evolution," to appear in J. Geometry and Physics.

(3) T. Brocker and T. tom Dieck, "Representations of Compact Lie Groups," SpringerVerlag (1985).

(4) Y. Choquet-Bruhat, C. DeWitt-Morette, and M. Dillard-Bleick, "Analysis, Manifolds and Physics," Vol. 1, North-Holland (1989).

(5) N. Steenrod, "The Topology of Fibre Bundles," Princeton University Press (19XX).

(6) M. Narasimhan and S. Ramanan, Amer. J. Math. 83, 563 (1961). 

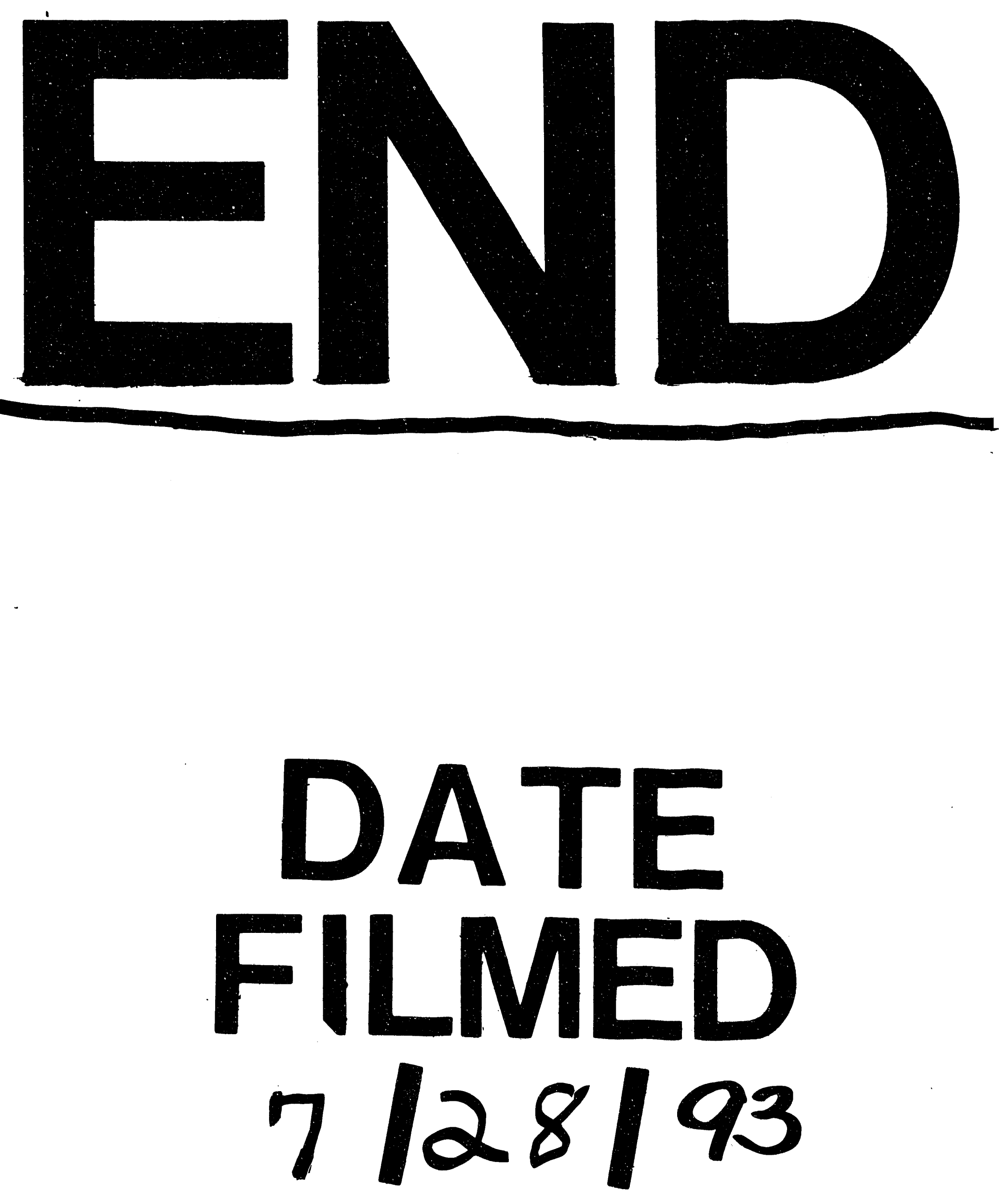

1 
